Fórmulas de Poincaré-Hopf e classes características de variedades singulares

Giuliano Angelo Zugliani 


\title{
Fórmulas de Poincaré-Hopf e classes características de variedades singulares*
}

\author{
Giuliano Angelo Zugliani
}

Orientadora: Profa. Dra. Maria Aparecida Soares Ruas

Dissertação apresentada ao Instituto de Ciências Matemáticas

e de Computação - ICMC-USP, como parte dos requisitos para obtenção do título de Mestre em Ciências - Matemática.

VERSÃO REVISADA APÓS A DEFESA

Data da Defesa: 08/02/2008

Visto da Orientadora:

USP - São Carlos

Fevereiro/2008

*Projeto financiado pela FAPESP. 


\section{Agradecimentos}

Este trabalho é dedicado, em primeiro lugar, a Deus, por tudo.

Aos meus pais, pela confiança e pelo esforço, e a toda minha família.

A minha querida Rosana, por todos os momentos juntos.

A todos os meus colegas de ICMC, tanto na pós-graduação, como na graduação, em especial aos meus irmãos Eduardo e Thiago.

Aos meus orientadores Cidinha, Daniel e Ton, com os quais foi muito bom trabalhar, e a todos os meus ex-professores, desde os níveis mais básicos. 



\section{Resumo}

Neste trabalho, estudamos diferentes construções e propriedades das classes características de variedades suaves e singulares. Para ilustrar a teoria, calculamos a obstrução de Euler de algumas superfícies singulares no espaço tridimensional e apresentamos uma fórmula do tipo Poincaré-Hopf para variedades singulares. 



\section{Abstract}

In this work, we study different constructions and properties of the characteristics classes of smooth and singular manifolds. To ilustrate the theory, we compute the Euler obstructions of some singular surfaces in tridimensional space and state a Poincaré-Hopf's formula for singular varieties. 



\section{Sumário}

Prefácio ........................

1 Requisitos básicos . . . . . . . . . . . . . . . . 1

1.1 Variedades . . . . . . . . . . . . . . . . . 1

1.2 Fibrados vetoriais . . . . . . . . . . . . . . . . . 3

1.3 Um pouco de cohomologia . . . . . . . . . . . . . 5

1.3.1 Cohomologia singular . . . . . . . . . . . . . . 6

2 O teorema de Poincaré-Hopf: versão suave . . . . . . . . . . . 11

2.1 O grau de Brower de uma aplicação . . . . . . . . . . . . . . . . . . 11

2.2 Índice de campos vetoriais . . . . . . . . . . . . . . . . . . . 13

2.3 A característica de Euler-Poincaré . . . . . . . . . . . . . . . . . . 14

2.3.1 Relação com os índices de um campo de vetores . . . . . . . . 15

3 Classes de Stiefel-Whitney . . . . . . . . . . . . . . 19

3.1 Classes características de Stiefel-Whitney . . . . . . . . . . . . . . . 19

3.2 Aplicação em imersões . . . . . . . . . . . . . . . . . . . . 20

3.3 Variedades de Grassmann . . . . . . . . . . . . . . . . . . . 20

4 Classe de Euler e aplicações . . . . . . . . . . . . . . . 23

4.1 Definição e propriedades básicas . . . . . . . . . . . . . . . . 23

4.2 Seqüência de Gysin de um fibrado vetorial . . . . . . . . . . . . . . 25

4.2.1 O fibrado universal orientável . . . . . . . . . . . . . . 26

4.3 Os fibrados normal e tangente . . . . . . . . . . . . . . 27

4.4 Aplicações . . . . . . . . . . . . . . . . . . . . . . . . . . . . . . 29

5 Classes de Chern ..................... 35

5.1 Variedades e fibrados complexos . . . . . . . . . . . . . . 35

5.2 Construção das classes de Chern . . . . . . . . . . . . . . . 36

5.3 Variedades de Grassmann complexas . . . . . . . . . . . . . . . 37

5.4 Fibrados duais (conjugados) . . . . . . . . . . . . . . . . . 40 
6 Obstruções . . . . . . . . . . . . . . . . . . 43

6.1 Definição das classes de Chern por obstrução . . . . . . . . . . . . . . 44

6.2 Relação com as classes de Stiefel-Whitney . . . . . . . . . . . . . . . 45

6.3 Relação com a classe de Euler . . . . . . . . . . . . . . . . . . . 46

7 Estratificações de Whitney . . . . . . . . . . . . 49

7.1 Conjuntos Algébricos . . . . . . . . . . . . . . . . . . . . . 49

7.2 Conjuntos Semi-algébricos . . . . . . . . . . . . . . 52

8 Classes de Schwartz . . . . . . . . . . . . . 57

8.1 Células duais . . . . . . . . . . . . . . . . . . . . . . 57

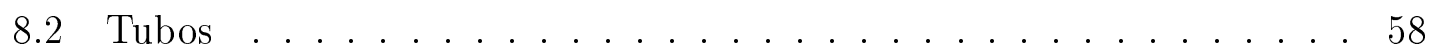

8.2 .1 Tubos celulares . . . . . . . . . . . . . . 58

8.2 .2 Tubos geodésicos . . . . . . . . . . . . . . . 59

8.3 Prolongamentos . . . . . . . . . . . . . . . . . 6 60

8.3.1 Prolongamento por paralelismo . . . . . . . . . . 60

8.3.2 Campos transversais . . . . . . . . . . . . . . 61

8.3.3 Prolongamento radial local . . . . . . . . . . . . . 63

8.4 Construção das classes de Schwartz . . . . . . . . . . . . . . . . . 63

9 Obstrução de Euler e classes de MacPherson . . . . . . . . . . 67

9.1 Conjectura de Deligne-Grothendieck . . . . . . . . . . . . . . . 67

9.2 A obstrução local de Euler . . . . . . . . . . . . . . . . . . . 69

9.2.1 Outra forma de construção . . . . . . . . . . . . . . . 71

9.3 Classe de Mather . . . . . . . . . . . . . . . . . . . . . . . . . 72

9.4 Cálculos de obstruções . . . . . . . . . . . . . . . . . . . . . . . 74

10 O teorema de Poincaré-Hopf: versão singular $\ldots \ldots$. . . . . 79

Referências Bibliográficas ................. 83

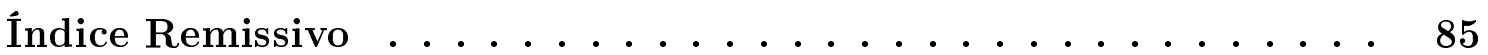




\section{Lista de Figuras}

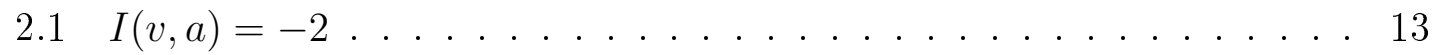

2.2 Identificação dos lados na construção do toro pinçado . . . . . . . . . 15

2.3 Toro pinçado . . . . . . . . . . . . . . . . . 15

2.4 Campo de Hopf . . . . . . . . . . . . . . . . . 16

2.5 Campos na esfera e no toro . . . . . . . . . . . . 17

4.1 Vizinhança tubular de $M \ldots \ldots \ldots \ldots$. . . . . . . . . . . . . .

6.1 Estendendo ao interior de $\sigma \ldots \ldots \ldots$. . . . . . . . . . . 44

6.2 Seção constante sobre $\partial \sigma \ldots \ldots$. . . . . . . . . . . . . . . 46

7.1 Guarda-chuva de Whitney . . . . . . . . . . . . . . 50

8.1 Células duais . . . . . . . . . . . . . . . . . . . . . . 58

8.2 Tubo celular . . . . . . . . . . . . . . . . . . . 59

8.3 Tubo geodésico . . . . . . . . . . . . . . . . . 60

8.4 Campo radial prolongado . . . . . . . . . . . . . . . 61

8.5 Campo não-estratificado . . . . . . . . . . . . . . . . . . . . 62

8.6 Vizinhança prismática . . . . . . . . . . . . . . . . 62

8.7 Construção do campo radial . . . . . . . . . . . . . . . . 64

9.1 Modificação de Nash . . . . . . . . . . . . . . . . . . . . 70

9.2 Obstrução à extensão de $\tilde{v}$ sobre $\nu^{-1}(\Theta) \ldots \ldots$. . . . . . . . . . 72

9.3 Membro da família $S_{k} \ldots \ldots \ldots \ldots$. . . . . . . . . . 75

9.4 Membro da família $B_{k} \ldots \ldots \ldots \ldots 76$

10.1 Campos no toro pinçado . . . . . . . . . . . . . . . . . 79

10.2 Campo na figura $8 \ldots \ldots \ldots$. . . . . . . . . . . . . . 81 



\section{Prefácio}

Em 1935, Stiefel e Whitney construíram, independentemente, classes características para variedades suaves, como obstrução à construção de seções do fibrado tangente. Mas a primeira noção de obstrução já havia aparecido com Poincaré.

Em 1758, Euler obteve a famosa relação entre vértices, arestas e faces de um poliedro de dimensão 2 em $\mathbb{R}^{3}$. Poincaré generalizou este resultado em 1893 para poliedros finitos de dimensão $n$ em $\mathbb{R}^{m}$, definindo assim o invariante que hoje é denominado característica de Euler-Poincaré. Antes, em 1885, ele já havia demonstrado, para dimensão 2, o que seria o teorema de Poincaré-Hopf (Hopf, em 1927, o generalizou para as demais dimensões). Este teorema estabelece uma relação entre a característica de Euler-Poincaré de uma variedade suave $M$ e as singularidades de um campo de vetores tangentes a $M$, calculando assim a obstrução à construção de campos não-nulos na variedade. A característica de Euler-Poincaré é, então, o primeiro exemplo de classe característica. Apresentar este resultado é o objetivo do capítulo 2.

A partir daí, construiremos algumas classes caracterísicas e apresentaremos suas propriedades básicas, iniciando com variedades suaves (tomando como base o livro de J. Milnor [18]).

No capítulo 3, falaremos das classes de Stiefel-Whitney (com coeficientes em $\mathbf{Z}_{2}$ ), mas do ponto de vista axiomático, onde os cálculos são mais simples de serem computados. No capítulo 4, apresentaremos a classe de Euler e aplicações deste invariante. No capítulo 5, serão construídas as classes de Chern de fibrados vetoriais complexos, obtidas através da seqüência de Gysin.

As referências principais de cada capítulo aparecem no ínicio dos mesmos. Algumas demonstrações de resultados foram omitidas, ora por simplicidade das mesmas, ora por conveniência. Demos maior relevância às demonstrações mais importantes no contexto, e àquelas em que acreditamos ter contribuído com algum detalhe, por menor que seja.

O estudo das classes no caso suave termina no capítulo 6, de obstruções. Da mesma maneira que o teorema de Poincaré-Hopf, esta teoria define ferramentas que 
medem a obstrução para a construção de um dado número de seções em um fibrado. Construiremos a classe de Chern desta forma, que é mais geométrica (mas mais difícil de calcular) e apresentaremos sua relação com as classes já construídas. Além disso, este será o ponto natural de indagarmos sobre o caso singular. Iniciamos este com o estudo de estratificações, no capítulo 7 .

O ponto crucial é que agora o fibrado tangente não existe mais. Para generalizá-lo, iremos considerar a união dos fibrados tangentes aos estratos de uma estratificação da variedade. É assim que M.H. Schwartz, em 1965, apresentou uma construção de campos radiais e, a partir deles, de classes características de variedades singulares. Usando o artigo [1] como referência, no capítulo 8, estudaremos estas classes, apresentando as definições de tubos e prolongamentos, pré-requisitos ao entendimento das mesmas. Esta construção é bem geométrica e, num certo sentido, estende a definição de classes de Chern dada no capítulo 6.

Uma outra forma de construção das classes de Chern no caso singular é feita por R. MacPherson, em 1974, no artigo [15]. Esta é mais axiomática, pois aparece como prova da conjectura de Deligne-Grothendieck, sobre a existência e unicidade das classes características de variedades singulares. Este é o objetivo do capítulo 9. Para tal, também construiremos a obstrução de Euler (também de duas maneiras, uma delas mais geométrica) e a calcularemos em dois exemplos de variedades singulares.

No capítulo 10, voltamos ao teorema central, no caso singular. Agora, o teorema de Poincaré-Hopf não é mais verdadeiro. Necessitaremos do campo radial de M.H. Schwartz para recuperar este resultado.

Ressaltamos que a teoria de classes características de variedades singulares tem alcançado um grande impulso atualmente. Diversos pesquisadores têm dado contribuição fundamental ao assunto, estendendo em diversas direções os trabalhos aqui referidos. 


\section{Capítulo 1}

\section{Requisitos básicos}

O objetivo deste capítulo de pré-requisitos é fixar notações utilizadas no texto, bem como enunciar os resultados básicos mais importantes. Referimo-nos sempre às referências para maiores detalhes e demonstrações.

Apresentaremos aqui noções básicas de variedades, definições e resultados sobre fibrados, um pouco de cohomologia e suas operações. Um conhecimento básico de homologia será assumido.

\section{$1.1 \quad$ Variedades}

A referência para os detalhes é [16].

Definição 1.1. Sejam $X \subset \mathbb{R}^{k}$ e $Y \subset \mathbb{R}^{l}$; uma função $f: X \rightarrow Y$ é suave se para cada $x \in X$ existe um aberto $U \subset \mathbb{R}^{k}$ e uma função $F: U \rightarrow \mathbb{R}^{l}$ suave, tal que $F(a)=f(a)$, para todo $a \in X \cap U$.

Notamos que, com esta definição, a composição de aplicações suaves ainda é suave. Dizemos que $X$ é difeomorfo a $Y$ se existe uma função $f: X \rightarrow Y$ suave com inversa suave.

Definição 1.2. Um subconjunto $M \subseteq \mathbb{R}^{k}$ é denominado uma variedade suave de dimensão $m$ se cada $x \in M$ possui uma vizinhança em $M$ difeomorfa a um aberto $U \subseteq \mathbb{R}^{m}$

Vamos agora definir os planos tangentes a uma variedade suave. Inicialmente, para um aberto $U \subseteq \mathbb{R}^{k}$, definimos o plano tangente a $U$ em $x$ como $T_{x} U \doteq \mathbb{R}^{k}$.

Seja agora $f: U \rightarrow V$ suave, com $V \subseteq \mathbb{R}^{l}$. A derivada de $f$ em $x \in U$, $d_{x} f: \mathbb{R}^{k} \rightarrow \mathbb{R}^{l}$, é a transformação linear cuja matriz é a matriz Jacobiana de $f$ em $x$. 
Valem aqui:

(i) (A Regra da cadeia) Se $f: U \rightarrow V$ e $g: V \rightarrow W$ são suaves (com $U$ e $V$ abertos em seus respectivos espaços), então $d_{x}(g \circ f)=d_{f(x)} g \circ d_{x} f$.

(ii) Se $U_{1}$ é um aberto de $\mathbb{R}^{k}, U_{2}$ é um aberto de $U_{1}$, e $i: U_{2} \rightarrow U_{1}$ é a aplicação de inclusão, então para todo $x \in U_{2}, d_{x} i$ é a identidade de $\mathbb{R}^{k}$.

(iii) Se $L: \mathbb{R}^{k} \rightarrow \mathbb{R}^{l}$ é uma transformação linear, então para todo $x \in \mathbb{R}^{k}$, temos $d_{x} L=L$.

Como conseqüencia imediata da Regra da Cadeia, segue a proposição:

Proposição 1.3. Sejam $U$ e $V$ abertos de $\mathbb{R}^{k}$ e $\mathbb{R}^{l}$, respectivamente, e $f: U \rightarrow V$ um difeomorfismo. Então $d_{x} f: \mathbb{R}^{k} \rightarrow \mathbb{R}^{l}$ é não-singular, para todo $x \in U$.

Uma tentativa de recíproca deste resultado é o teorema da função inversa.

Proposição 1.4 (Teorema da função inversa). Sejam $U$ e $V$ abertos de $\mathbb{R}^{k}$, e $f: U \rightarrow V$ uma aplicação suave. Se existe $x \in U$ tal que $d_{x} f: \mathbb{R}^{k} \rightarrow \mathbb{R}^{k}$ é não -singular, então existe uma vizinhança $U^{\prime}$ de $x$ que é difeomorfa a sua imagem $f\left(U^{\prime}\right)$.

Seja agora $M \subseteq \mathbb{R}^{k}$ uma variedade suave de dimensão $m$. Dado $x \in M$, escolha $g: U \rightarrow M, U$ aberto, $U \subseteq \mathbb{R}^{m}$; temos que, se $g(u)=x, d_{u} g$ é uma transformação linear de $\mathbb{R}^{m}$ em $\mathbb{R}^{k}$ e assim, definimos $T_{x} M \doteq \operatorname{Im}\left(d_{u} g\right) \subseteq \mathbb{R}^{k}$.

Esta definição de plano tangente independe da aplicação $g$ escolhida (parametrização).

Uma outra caracterização de um vetor tangente a $M$ em $x$ é aquela que diz que o mesmo pode ser expresso como vetor velocidade de um caminho suave por $x$ $\operatorname{em} M(\gamma:(0,1) \rightarrow M)$.

Temos que $T_{x} M$ é um subespaço vetorial de $\mathbb{R}^{k}$ e sua dimensão é $m$. Podemos agora definir as derivadas de uma aplicação suave entre variedades $f: M \rightarrow N$. A derivada de $f$ em $x, d_{x} f: T_{x} M \rightarrow T_{f(x)} N$ é construída da seguinte forma. Seja $W$ um aberto do espaço ambiente $\mathbb{R}^{k}$ de $M$, contendo $x$, tal que temos $F$ como aplicação de $W$ no espaço ambiente $\mathbb{R}^{l}$ de $N$, suave e coincidindo com $f$ em $W \cap M$.

Colocamos então $d_{x} f(v)=d_{x} F(v)$, para todo $v \in T_{x} M \subseteq \mathbb{R}^{k}$ (a boa definição é mais uma conseqüência da Regra da Cadeia). 


\subsection{Fibrados vetoriais}

Nesta seção, a referência utilizada é [18].

Definição 1.5. Um fibrado vetorial real $\xi$ de dimensão $n$ sobre um espaço topológico $B$ (denominado base) consiste de um espaço topológico $E$ (espaço total) e de uma projeção $\pi: E \rightarrow B$ tal que todo ponto $b \in B$ admite uma vizinhança $U$ e um homeomorfismo $h: \pi^{-1}(U) \rightarrow U \times \mathbb{R}^{n}$ (trivialização local). Além disso, para todo $b \in B, \pi^{-1}(b)$ é um espaço vetorial real, e a restrição de $h$ a $\pi^{-1}(b)$ é um isomorfismo sobre $b \times \mathbb{R}^{n}$.

Um fibrado vetorial de dimensão $n$ freqüentemente será mencionado como $n$-fibrado.

O espaço vetorial $\pi^{-1}(b)$ é denominado fibra sobre $b \in B$ e também é denotado por $F_{b}(\xi)$.

Quando a vizinhança $U$ da trivialização local pode ser tomada como todo o espaço $B$, então o fibrado é dito trivial.

Definição 1.6. Dois fibrados $\xi$ e $\eta$, com mesma base $B$, são isomorfos se existe um homeomorfismo $f: E(\xi) \rightarrow E(\eta)$ entre os espaços totais tal que a fibra $F_{b}(\xi)$ é isomorfa a $F_{b}(\eta)$ por $f$.

Definição 1.7. Uma aplicação entre fibrados $\xi$ de base $B$ e $\eta$ de base $B^{\prime}$ é uma aplicação $f: E(\xi) \rightarrow E(\eta)$ onde cada fibra $F_{b}(\xi)$ é isomorfa por $f$ a alguma fibra $F_{b^{\prime}}(\eta)$.

Seja $\xi$ um fibrado sobre $B$ e seja $B^{\prime}$ um espaço topológico. Dada uma aplicação contínua $f: B^{\prime} \rightarrow B$, podemos construir um novo fibrado, induzido por $f$, sobre $B^{\prime}$ e denotado por $f^{*}(\xi)$.

O espaço total deste fibrado consiste dos pontos de $E^{\prime} \subset B^{\prime} \times E(\xi)$ onde $\left(b^{\prime}, e\right) \in E^{\prime}$ se, e somente se, $f\left(b^{\prime}\right)=\pi(e)$. Sua projeção consiste da aplicação $\pi^{\prime}: E^{\prime} \rightarrow B^{\prime}$ onde $\pi^{\prime}\left(b^{\prime}, e\right)=b^{\prime}$. Teremos então o seguinte diagrama comutativo:

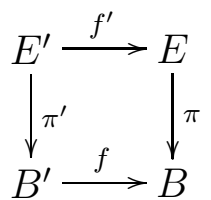

onde $f^{\prime}\left(b^{\prime}, e\right)=e$. Segue então que $f^{\prime}$ será uma aplicação entre os fibrados $f^{*}(\xi)$ e $\xi$.

Dados dois fibrados $\xi_{1}$ e $\xi_{2}$, com suas respectivas bases $B_{1}$ e $B_{2}$, podemos construir um fibrado (produto) sobre $B_{1} \times B_{2}$ onde a fibra sobre $\left(b_{1}, b_{2}\right)$ é o produto cartesiano $F_{b_{1}}\left(\xi_{1}\right) \times F_{b_{2}}\left(\xi_{2}\right)$. Este fibrado será denotado por $\xi_{1} \times \xi_{2}$. 
Se a base destes fibrados é a mesma, também definimos o fibrado soma de Whitney sobre $B$. Este será o fibrado induzido $\Delta^{*}\left(\xi_{1} \times \xi_{2}\right)$ onde $\Delta: B \rightarrow B \times B$ é a aplicação diagonal. A importante propriedade deste fibrado, denotado por $\xi_{1} \oplus \xi_{2}$, é de que sua fibra $F_{b}\left(\xi_{1} \oplus \xi_{2}\right)$ é a soma direta $F_{b}\left(\xi_{1}\right) \oplus F_{b}\left(\xi_{2}\right)$.

Exemplo 1.8. 1. O fibrado tangente $\tau_{M}$ é o fibrado com base sendo uma variedade suave $M$, espaço total $T M=\left\{(x, v): x \in M, v \in T_{x} M\right\}$ e projeção canônica.

2. O fibrado normal $\nu_{M}$ também é um fibrado com base $M \subset \mathbb{R}^{N}$ e espaço total $E \subset M \times \mathbb{R}^{N}$, onde $(x, v) \in E$ se, e somente se, $v \perp T_{x} M$. A projeção é definida canonicamente.

3. Construiremos o fibrado de retas sobre o espaço projetivo $P^{n}$ (variedade de dimensão $n$ ), denotado por $\gamma_{n}^{1}$. Seu espaço total consistirá de um subconjunto de $P^{n} \times \mathbb{R}^{n+1}$ onde $(\{ \pm x\}, v) \in E\left(\gamma_{n}^{1}\right)$ se, e somente se, $v$ é múltiplo de $x$. A projeção também é definida canonicamente. A fibra em cada ponto $\{ \pm x\}$ de $P^{n}$ é a reta em $\mathbb{R}^{n+1}$ que passa por estes pontos. Pode-se mostrar que este é um exemplo de fibrado não-trivial.

Definição 1.9. Uma seção de um fibrado $\xi$ com base $B$ é uma função contínua $s: B \rightarrow E(\xi)$ tal que $s(b) \in F_{b}(\xi)$.

Uma seção é dita não-nula se, para todo $b \in B$, o vetor $s(b)$ é diferente do elemento neutro do espaço vetorial $F_{b}(\xi)$.

Seções $s_{1}, \ldots, s_{n}$ são ditas linearmente independentes se, para todo $b \in B$, os vetores $s_{1}(b), \ldots, s_{n}(b)$ são linearmente independentes em $F_{b}(\xi)$.

Proposição 1.10. Um n-fibrado $\xi$ é trivial se, e somente se, admite $n$ seções linearmente independentes.

Definição 1.11. Um fibrado euclidiano constitui-se de um fibrado $\xi$ munido de uma aplicação contínua $\mu: E(\xi) \rightarrow \mathbb{R}$ (métrica euclidiana) tal que a restrição de $\mu$ a cada fibra $F_{b}(\xi)$ é uma norma para esse espaço vetorial.

No caso do fibrado tangente $\tau_{M}$ a uma variedade $M$, uma métrica euclideana é chamada métrica riemanniana, e $M$ é dita uma variedade riemanniana.

Dado um subfibrado $\xi$ de $\eta$ (isto é, $\xi$ e $\eta$ têm mesma base $B$, mas as fibras de $\xi$ são subespaços das de $\eta$ ), se $\eta$ possui métrica euclideana, então existe um fibrado sobre $B$, denotado por $\xi^{\perp}$, tal que $\xi \oplus \xi^{\perp}=\eta$. Para o caso de fibrados tangentes, temos: 
Proposição 1.12. Para qualquer subvariedade $M$ de uma variedade riemanniana $N$, temos:

$$
\tau_{M} \oplus \nu_{M}=\tau_{N \mid M}
$$

(onde $\tau_{N \mid M}$ indica restrição do fibrado $\tau_{N}$ a $M$ ).

\subsection{Um pouco de cohomologia}

Neste resumo, pretendemos apresentar os resultados da teoria de cohomologia mais usados durante o texto, enfatizando o caráter dual que ela possui com a teoria de homologia. Para maior detalhamento, as referências utilizadas são [21] e [25].

Definição 1.13. Uma Categoria consiste de uma família de objetos $X$ e de um conjunto de morfismos hom $(A, B)$, para cada par $(A, B)$ de objetos. Está definida uma composição entre os morfismos satisfazendo a associatividade e a existência do elemento neutro.

Definição 1.14. Um funtor contravariante entre duas categorias $\mathcal{C}, \mathcal{D}$ é uma função que associa a cada objeto $A$ de $\mathcal{C}$, um objeto $G(A)$ de $\mathcal{D}$ e a cada morfismo $f: A \rightarrow B$ de $\mathcal{C}$ um morfismo $G(f): G(B) \rightarrow G(A)$ de $\mathcal{D}$ tal que, se $1_{\mid A}$ indica a função identidade de $A$,

$$
G\left(1_{\mid A}\right)=1_{\mid G(A)}
$$

e

$$
G(g \circ f)=G(f) \circ G(g)
$$

Observação 1.15. Se, nas mesmas condições, o morfismo associado a $f$ é $G(f)$ : $G(A) \rightarrow G(B)$, temos um funtor covariante. Um exemplo deste tipo de funtor é aquele que associa, a cada aplicação contínua entre espaços topológicos $A$ e $B$ o homomorfismo induzido $f_{*}: H_{p}(A) \rightarrow H_{p}(B)$ entre os $p$-ésimos grupos de homologia.

Exemplo 1.16 (O funtor Hom). Considere $\mathcal{C}$ a categoria dos grupos abelianos. Constrói-se um funtor contravariante dessa categoria nela própria, da seguinte forma. Fixe um objeto $G$ de $\mathcal{C}$; para cada objeto $A$ de $\mathcal{C}$, associamos $\operatorname{Hom}(A, G)=\{$ homomorfismos de $A$ em $G\}$, um novo grupo abeliano. A cada homomorfismo $f: A \rightarrow B$ de $\mathcal{C}$, associamos o novo homomorfismo $\tilde{f}: \operatorname{Hom}(B, G) \rightarrow \operatorname{Hom}(A, G)$ definido por: 


$$
\tilde{f}(\phi)=\phi \circ f
$$

para todo $\phi \in \operatorname{Hom}(B, G)$.

Uma propriedade simples e útil do funtor Hom é a seguinte

Lema 1.17. (i) $\operatorname{Hom}\left(\oplus_{j=1}^{n} A_{j}, G\right) \simeq \oplus_{j=1}^{n} \operatorname{Hom}\left(A_{j}, G\right)$;

(ii) $\operatorname{Hom}\left(A, \oplus_{j=1}^{n} G_{j}\right)=\oplus_{j=1}^{n} \operatorname{Hom}\left(A, G_{j}\right)$.

\subsubsection{Cohomologia singular}

Definição 1.18. Um simplexo $s$ de dimensão $n$ é o menor subconjunto convexo de $\mathbb{R}^{m}$ que contém os pontos $a_{0}, \ldots, a_{n} \in \mathbb{R}^{m}$ (vértices), onde $a_{1}-a_{0}, \ldots, a_{n}-a_{0}$ são linearmente independentes. Uma face de um simplexo é qualquer simplexo que tenha por vértices um subconjunto do conjunto de vértices de $s$.

Um simplexo de dimensão $n$ em $\mathbb{R}^{m}$ será denotado por $\Delta_{n}$. Um $n$-simplexo num espaço topológico $X$ é uma função contínua $\xi: \Delta_{n} \rightarrow X$. O conjunto destas aplicações será denotado por $S_{n}(X)$ e o grupo abeliano livre gerado por $S_{n}(X)$ será denotado por $C_{n}(X)$. Na teoria de homologia, define-se o operador bordo $\partial_{n}: C_{n}(X) \rightarrow C_{n-1}(X)$ tal que $\partial \circ \partial=0$ e definem-se a partir daí os grupos de homologia.

Aqui, em cohomologia, faremos um procedimento análogo (ou melhor, dual) utilizando o funtor Hom. Definimos o grupo de cocadeias de $X$ por $C^{n}(X) \doteq$ $\operatorname{Hom}\left(C_{n}(X), G\right)$, onde $G$ é um grupo abeliano fixado.

Por sua vez, o operador cobordo é definido como dual do operador bordo e denotado por $\delta^{n}: C^{n-1}(X) \rightarrow C^{n}(X)$.

A partir daí, definimos os cociclos como $Z^{n}(X, G) \doteq k e r\left(\delta^{n+1}\right)$ e os cobordos como $B^{n}(X, G) \doteq \operatorname{Im}\left(\delta^{n}\right)$. Assim, utilizando o operador cobordo, aqui também teremos $Z^{n}(X, G) \subseteq B^{n}(X, G)$ e podemos definir o $n$-ésimo grupo de cohomologia

$$
H^{n}(X, G)=\frac{Z^{n}(X, G)}{B^{n}(X, G)}
$$

Seja $c^{n}$ uma cocadeia de $X$. Denotamos a ação desta cocadeia em $c_{n} \in C_{n}(X)$ por $<c^{n}, c_{n}>\in G$. Com esta notação, para todo elemento $d_{n+1} \in C_{n+1}(X)$, temos

$$
<\delta^{n+1}\left(c^{n}\right), d_{n+1}>=<c^{n}, \partial_{n+1}\left(d^{n+1}\right)>.
$$

Agora seja $A$ um subconjunto de $X$. Define-se, em homologia o grupo quociente $C_{n}(X, A)$. O seu dual será denotado por $C^{n}(X, A, G)$. O operador bordo, 
quocientado, dará origem a um novo operador cobordo e, de maneira análoga à feita acima, construimos os grupos de cociclos e de cobordos relativos $\left(Z^{n}(X, A, G)\right.$ e $B^{n}(X, A, G)$, respectivamente) e daí teremos

$$
H^{n}(X, A, G)=\frac{Z^{n}(X, A, G)}{B^{n}(X, A, G)}
$$

que são os grupos de cohomologias relativos a $A$.

Em homologia, a cada aplicação de pares $f:(X, A) \rightarrow(Y, B)(f(A) \subset B)$, existe uma aplicação induzida $f_{n}: C_{n}(X, A) \rightarrow C_{n}(Y, B)$ tal que $f_{n} \circ \partial_{n+1}=\partial_{n} \circ f_{n}$ (o par $(X, \emptyset)$ sempre será abreviado para $X$ ). A aplicação dual de $f_{n}$, denotada por $f^{n}$, então, terá a propriedade $f^{n} \circ \delta_{n}=\delta_{n+1} \circ f^{n}$.

Esta aplicação $f^{n}$ preserva cociclos e, daí podemos definir por passagem ao quociente a aplicação induzida em cada nível de cohomologia $f^{*}: H^{n}(Y, B, G) \rightarrow$ $H^{n}(X, A, G)$.

A cohomologia singular, assim construída, satisfaz os seguintes axiomas da teoria de cohomologia, e a propriedade listada a seguir, que serão freqüentemente utilizadas no decorrer do texto.

Axioma 1.19. A aplicação induzida pela identidade entre pares de espaços topológicos é a identidade entre os grupos de cohomologia.

Axioma 1.20. Se $f:(X, A) \rightarrow(Y, B)$ e $g:(Y, B) \rightarrow(Z, C)$ são aplicações continuas entre pares de espaços topológicos, então $(g \circ f)^{*}=f^{*} \circ g^{*}$.

Axioma 1.21. Se incl denota a aplicação inclusão e j a projeção no quociente entre cocadeias, a seguinte seqüência é exata:

$$
\cdots \longrightarrow H^{p-1}(A, G) \longrightarrow H^{p}(X, A, G) \stackrel{j^{*}}{\longrightarrow} H^{p}(X, G) \stackrel{(i n c l)^{*}}{\longrightarrow} H^{p}(A, G) \longrightarrow \cdots
$$

Axioma 1.22. As aplicações induzidas por $f:(X, A) \rightarrow(Y, B)$ tornam o seguinte diagrama comutativo, em cada nível de cohomologia:

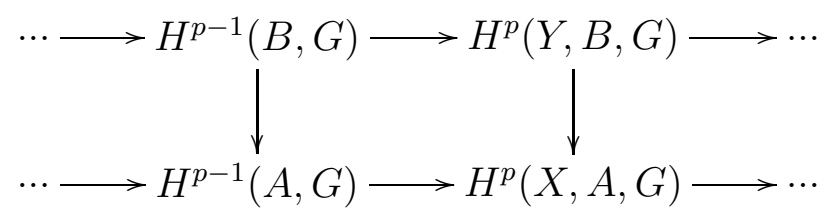

Axioma 1.23. Se $f$ e $g$ como acima são homotópicas, então $f^{*}=g^{*}$ 
Axioma 1.24 (Excisão). Seja $U$ um aberto de $X$ tal que $\bar{U} \subset$ int $A$. Então a inclusão do par $(X-U, A-U)$ em $(X, A)$ induz um isomorfirsmo $H^{p}(X, A, G) \rightarrow$ $H^{p}(X-U, A-U, G)$.

Axioma 1.25. Se $X=\left\{p_{0}\right\}$ (isto é, $X$ consiste de apenas um ponto), então

$$
H^{p}(X, G)=\left\{\begin{array}{cc}
G, & p=0 \\
0, & p \neq 0
\end{array}\right.
$$

Proposição 1.26. Se $\left\{X_{\alpha}\right\}_{\alpha \in A}$ são as componentes conexas por caminhos de $X$, então

$$
H^{0}(X, G) \simeq \prod_{\alpha \in A} G_{\alpha}
$$

(isto é, é isomorfo ao produto direto de cópias de G)

\section{Operações importantes}

Três operações, denominadas produtos, envolvendo os grupos de cohomologia, serão muito úteis em nossos propósitos.

\section{(a) O produto cup}

Suponha agora que $R$ seja um anel com unidade. Coloquemos $S^{n}(X) \doteq$ $\operatorname{Hom}\left(S_{n}(X), R\right)$. Definimos um produto

$$
S^{n}(X) \times S^{m}(X) \stackrel{\cup}{\longrightarrow} S^{n+m}(X)
$$

através da seguinte equação. Seja $\Delta_{n+m}$ o $(n+m)$-simplexo com vértices $\left\{a_{0}, \ldots, a_{n+m}\right\}$. Se $T: \Delta_{n+m} \rightarrow X$ é um $(n+m)$-simplexo em $X$, então

$$
<c^{n} \cup c^{m}, T>=<c^{n}, T_{\mid \Delta_{n}}>\cdot<c^{m}, T_{\mid \Delta_{m}}>,
$$

onde o produto é o produto do anel $R . \Delta_{n}$ aqui será a face de $\Delta_{n+m}$ constituída dos vértices $\left\{a_{0}, \ldots, a_{n}\right\}$ e $\Delta_{m}$ será a face de $\Delta_{n+m}$ constituída dos vértices $\left\{a_{n}, \ldots, a_{n+m}\right\}$.

Esta operação induz uma operação nos grupos de cohomologia, denominada produto cup:

$$
H^{n}(X, R) \times H^{m}(X, R) \stackrel{\cup}{\longrightarrow} H^{n+m}(X, R) .
$$

Esta operação é bilinear, associativa, anticomutativa e é preservada pelas aplicações induzidas em cohomologia. 
Outro importante fato é que o produto direto de grupos $H^{*}(X, R)=$ $\prod_{n \in \mathbf{Z}} H^{n}(X, R)$ é um anel com a operação produto cup (com unidade $1 \in H^{0}(X, R)$ ), chamado anel de cohomologia de $X$.

Observação 1.27. O produto cup também pode ser generalizado a uma aplicação envolvendo os grupos de cohomologia relativos:

$$
H^{n}(X, A) \times H^{m}(X, B) \stackrel{\cup}{\longrightarrow} H^{n+m}(X, A \cup B) .
$$

\section{(b) O produto cap}

Definiremos agora uma nova operação produto

$$
C^{j}(X) \times C_{n}(X) \stackrel{\cap}{\longrightarrow} C_{n-j}(X)
$$

com a seguinte propriedade: para cada cocadeia $b \in C^{j}(X)$ e para cada cadeia $\xi \in C_{n}(X), b \cap \xi$ será o único elemento de $C_{n-j}(X)$ tal que $<a, b \cap \xi>=<a \cup b, \xi>$, para todo $a \in C^{n-j}(X)$.

Esta operação também induz uma operação

$$
H^{j}(X, R) \times H_{n}(X) \stackrel{\cap}{\longrightarrow} H_{n-j}(X)
$$

denominada produto cap.

\section{(c) O produto cruzado}

O último produto a ser definido será o produto cruzado:

$$
H^{m}(X, R) \times H^{n}(Y, R) \stackrel{\times}{\longrightarrow} H^{m+n}(X \times Y, R) .
$$

Para isso, considere as projeções:

$$
p_{1}: X \times Y \rightarrow X
$$

e

$$
p_{2}: X \times Y \rightarrow Y
$$

Então definimos

$$
a \times b \doteq p_{1}^{*}(a) \cup p_{2}^{*}(b) \in H^{m+n}(X \times Y, R) .
$$

Uma propriedade distributiva importante que relaciona o produto cup com o produto cruzado é a seguinte: 
Proposição 1.28. Se $a, c \in H^{*}(X, \mathbf{Z})$ e $b, d \in H^{*}(Y, \mathbf{Z})$, então

$$
(a \times b) \cup(c \times d)=(-1)^{\operatorname{dim}(b) \cdot \operatorname{dim}(c)}(a \cup c) \times(b \cup d) .
$$

Para a demonstração, vide [9], tópico (29.17). No caso de coeficientes em $\mathbf{Z}_{2}$, os sinais não são necessários.

Como exemplo de grupo de cohomologia de espaços topológicos, citamos o teorema a seguir. Ele ajudará no cálculo das classes características nos Capítulos 2 e 5 .

Teorema 1.29. $H^{m}\left(\mathbf{P}^{2}, \mathbf{Z}_{2}\right)$ é um grupo cíclico de ordem 2 , para $0 \leq m \leq n$, e nulo, para $m>n$. Mais ainda, se a denota o gerador de $H^{1}\left(\mathbf{P}^{2}, \mathbf{Z}_{2}\right)$, então a ${ }^{m}$ (potências do produto cup) é o gerador de $H^{m}\left(\mathbf{P}^{2}, \mathbf{Z}_{2}\right), 0 \leq m \leq n$ (e, portanto, $\left.a^{m+1}=0\right)$.

Para a demonstração, vide [23], pág. 264. 


\section{Capítulo 2}

\section{O teorema de Poincaré-Hopf: versão}

\section{suave}

Continuaremos introduzindo resultados gerais sobre variedades suaves, para o entendimento do cálculo de índices de campo de vetores. Definiremos a característica de Euler-Poincaré e apresentaremos o teorema central, no caso suave. A referência para este capítulo continua sendo [16].

\subsection{O grau de Brower de uma aplicação}

Definição 2.1. Seja $f: M \rightarrow N$ aplicação suave entre variedades de mesma dimensão. Um ponto $x \in M$ é um ponto regular se $d_{x} f$ tem posto máximo. $\mathrm{O}$ ponto $y \in N$ é um valor regular se $f^{-1}(y)$ consiste apenas de pontos regulares.

Se $M$ é compacta e $y$ é valor regular, então $f^{-1}(y)$ é vazio ou finito. De fato, $f^{-1}(y)$ é fechado num compacto, portanto, compacto e discreto, já que $f$ é bijetora em cada vizinhança de $x \in f^{-1}(y)$. Assim, podemos definir $\# f^{-1}(y)$.

Além disso, $\# f^{-1}(y)$ é localmente constante, como função de $y$. Para ver isto, basta considerar a seguinte vizinhança $V$ de $y: V=\left(V_{1} \cap V_{2} \cap \ldots \cap V_{k}\right)-f(M-$ $\left.U_{1}-U_{2}-\ldots-U_{k}\right)$, onde $x_{1}, x_{2}, \ldots, x_{k}$ são os pontos de $f^{-1}(y)$ e $U_{i}$ é uma vizinhança de $x_{i}$ difeomorfa a $V_{i}, i=1, \ldots, k, \operatorname{com} U_{i} \cap U_{j}=\emptyset, i \neq j$.

Definição 2.2. $X \subset \mathbb{R}^{k}$ é uma $m$-variedade com bordo se todo ponto $x \in X$ admite uma vizinhança difeomorfa a um aberto do semi-plano $H^{m}=\left\{\left(x_{1}, \ldots, x_{m}\right) \in \mathbb{R}^{m}\right.$ : $\left.x_{m} \geq 0\right\}$.

O espaço tangente a um ponto do bordo $\partial X$ (variedade suave de dimensão $m-1$ ) é definido como $T_{x} X \doteq \mathbb{R}^{m}$. 
Definição 2.3. Dado $X \subset \mathbb{R}^{k}$, duas aplicações $f, g: X \rightarrow Y$ suaves são homotópicas se existe $F: X \times[0,1] \rightarrow Y$ suave tal que $F(x, 0)=f(x)$ e $F(x, 1)=g(x), x \in X$.

Observamos que a homotopia é uma relação de equivalência.

Se $f$ e $g$, como acima, são difeomorfismos homotópicos e, além disso, $x \mapsto$ $F(x, t)$ é difeomorfismo, para todo $t \in[0,1]$, então $f$ e $g$ são aplicações isotópicas.

O seguinte resultado será muito importante:

Teorema 2.4 (§4, Homogeneidade). Suponha que $N$ é uma variedade conexa e que $y, z$ são pontos interiores de $N$. Então existe $h: N \rightarrow N$ difeomorfismo isotópico à identidade tal que $h(y)=z$.

Sabemos dar uma orientação a um n-espaço vetorial definindo uma relação de equivalência entre suas bases. Uma orientação a uma $m$-variedade $M$ é uma escolha de orientações para cada espaço tangente satisfazendo: para cada $x \in M$, existe parametrização $h: U \rightarrow M$ tal que $d_{u} h: \mathbb{R}^{m} \rightarrow T_{x} M$ preserva orientação para todo $u \in U$. Claramente uma orientação de uma variedade com bordo $X$ induz uma orientação em $\partial X$.

Sejam $M$ e $N$ variedades suaves, orientáveis e sem bordo, com $M$ compacta e $N$ conexa. Dada uma aplicação suave $f: M \rightarrow N$, sabemos que, para cada ponto regular $x \in M$, a derivada $d_{x} f: T_{x} M \rightarrow T_{f(x)} N$ é um isomorfismo, que pode preservar a orientação, ou revertê-la. No primeiro caso, colocamos sign $d_{x} f=1$, e no segundo, sign $d_{x} f=-1$. Para cada valor regular $y \in N$, definimos o grau de $f$ em $y$ como $\operatorname{deg}(f, y) \doteq \sum_{x \in f^{-1}(y)}$ sign $d_{x} f$. Pelo Lema de Sard ( $\S 3$ de [16]), o grau está definido em um subconjunto denso de $N$. Além disso, como as variedades são orientáveis, o grau de $f$ em $y$ é constante em uma vizinhança de $y$.

Lema 2.5 (§5, Lema 1). Suponha que a variedade $M$ é bordo de uma variedade suave, compacta e orientável $X$ e que $f: M \rightarrow N$ se estende a uma aplicação $F: X \rightarrow N$ suave. Então, para todo valor regular y de $N$, temos deg $(f, y)=0$

Através deste lema, pode-se mostrar o importante resultado a seguir:

Proposição 2.6 (§5, Lema 2). Suponha que y é valor regular de duas aplicações homotópicas $f, g: M \rightarrow N$. Então, $\operatorname{deg}(f, y)=\operatorname{deg}(g, y)$.

Esta proposição, por sua vez, nos permite concluir que o grau de $f$ independe do valor regular escolhido.

De fato, se $y$ e $z$ são valores regulares de $f$, escolha, pelo Teorema 2.4., um difeomorfismo $h: N \rightarrow N$ isotópico à identidade, com $h(y)=z$. Então $h$ preserva orientação e, usando a proposição acima, 


$$
\operatorname{deg}(f, y)=\operatorname{deg}(h \circ f, h(y))=\operatorname{deg}(h \circ f, z)=\operatorname{deg}(f, z) .
$$

Desta forma, denotaremos o grau da aplicação $f: M \rightarrow N$ apenas por $\operatorname{deg}(f)$.

\section{2 Índice de campos vetoriais}

Um campo vetorial em uma variedade suave $M$ é uma aplicação suave que a cada ponto $x \in M$ associa um vetor $v(x) \in T_{x} M$.

Suponha que $M$ tenha dimensão $m$ e seja $U$ uma bola de $\mathbb{R}^{m}$, centrada em um ponto $z$, difeomorfa a uma vizinhança em $M$. Suponha também que $v$ seja um campo suave em $U$, com zero isolado em $z$. Temos então que $\tilde{v}(x)=\frac{v(x)}{\|v(x)\|}$ leva uma esfera centrada em $z$ na esfera unitária de $\mathbb{R}^{m}$. O grau desta aplicação é chamado indice do campo $v$ em $z$ (ou no ponto correspondente na variedade $M$ ) e denotado por $I(v, z)$. Intuitivamente, o grau desta aplicação é o número de vezes que $S^{m-1}$ é recoberta (e é independente da escolha de $U$ ).

Segue um exemplo de cálculo de índice:

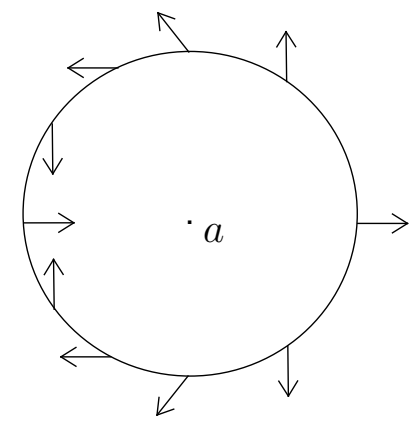

FIGURA 2.1: $I(v, a)=-2$

Suponha agora que $X$ é uma variedade suave de dimensão $m$, compacta, orientável e com bordo. A aplicação definida acima $g: \partial X \rightarrow S^{m-1}$ que, a cada $x \in \partial X$ associa o vetor unitário correspondente em $S^{m-1}$ apontando para fora, é chamada aplicação de Gauss .

Proposição 2.7 (Hopf). Se v é um campo vetorial definido em $X$, com zeros isolados $\left\{a_{j}\right\}$, e sempre apontando para fora ao longo do bordo, então a soma dos indices $\sum I\left(v, a_{j}\right)$ é igual ao grau da aplicação de Gauss. 
Demonstração. Removendo uma bola suficientemente pequena em torno de cada zero isolado, obteremos uma nova variedade $X^{\prime}$ com bordo. A aplicação $\tilde{v}(x)=\frac{v(x)}{\|v(x)\|}$ leva esta variedade em $S^{m-1}$. Além disso, como $\tilde{v}_{\mid \partial X}$ é homotópica a $g$, temos que o grau de $\tilde{v}_{\mid \partial X^{\prime}}$ é igual ao grau de $g$ (referente a componente $\partial X$ ), menos somatório dos índices. Mas o valor do grau de $\tilde{v}_{\mid \partial X^{\prime}}$ é nulo, pelo Lema 2.5.

Um resultado similar pode ser enunciado para uma variedade $M \subset \mathbb{R}^{k}$ compacta e sem bordo. Coloque $N_{\varepsilon}=\left\{x \in \mathbb{R}^{k}:\|x-y\| \leq \varepsilon\right.$, para algum $\left.y \in M\right\}$ a $\varepsilon$-vizinhança de $M$. Para $\varepsilon$ suficientemente pequeno, $N_{\varepsilon}$ é uma $k$-variedade com bordo.

Proposição 2.8 (§6, Teorema 1). Se v é um campo em $M$ com zeros isolados e não degenerados (isto é, $d_{z} v$ é não-singular), então a soma dos indices de $v$ é igual ao grau da função de Gauss $g: \partial N_{\varepsilon} \rightarrow S^{k-1}$.

\subsection{A característica de Euler-Poincaré}

Definição 2.9. Um poliedro $P$ em $\mathbb{R}^{m}$ é uma coleção finita de simplexos tais que:

(i) Toda face de um simplexo de $P$ deve ser um simplexo de $P$;

(ii) Se $s$ e $s^{\prime}$ são simplexos de $P$, a intersecção $s \cap s^{\prime}$ ou é vazia ou é uma face comum a $s$ e $s^{\prime}$.

Seja $a_{i}, i=1, \ldots, n$ o número de simplexos de dimensão $i$ de $P$. A característica de Euler-Poincaré de $P$ é definida como:

$$
\chi(P)=\sum_{i=1}^{n}(-1)^{i} a_{i} .
$$

Uma outra forma de se calcular a característica de Euler-Poincaré é através do seguinte teorema (vide [3] ou [12]) que a relaciona com a homologia de P. Este resultado é devido a Poincaré (1893):

Teorema 2.10 (Poincaré). Seja $P$ um Poliedro finito de dimensão $n$ em $\mathbb{R}^{m}$, com números de Betti $\beta_{i}(P)=\operatorname{dimH}_{i}(P, \mathbb{Z})$. Temos

$$
\chi(P)=\sum_{i=0}^{n}(-1)^{i} \beta_{i}(P) .
$$

Uma triangulação de um espaço topológico $X$ é um homeomofismo $h: P \rightarrow$ $X$, onde $P$ é um poliedro. Podemos definir assim característica de Euler-Poincaré de espaços topológicos. Alexander mostrou em 1915 que, se dois poliedros são 
isomorfos, então seus números de Betti são os mesmos, logo a característica de Euler-Poincaré independe da triangulação de um espaço topológico.

Como exemplos, sabemos que, como a característica de Euler Poincaré da cada poliedro de dimensão $2 \mathrm{em} \mathbb{R}^{3}$ é 2 , temos que $\chi\left(S^{2}\right)=2$. Uma triangulação natural do toro, obtida através da maneira usual de vê-lo, identificando os lados opostos de um retângulo, nos leva a $\chi\left(T^{2}\right)=0$. As Figuras 2.2 e 2.3 nos mostram uma triangulação do toro pinçado, uma variedade singular: sua característica vale 1.

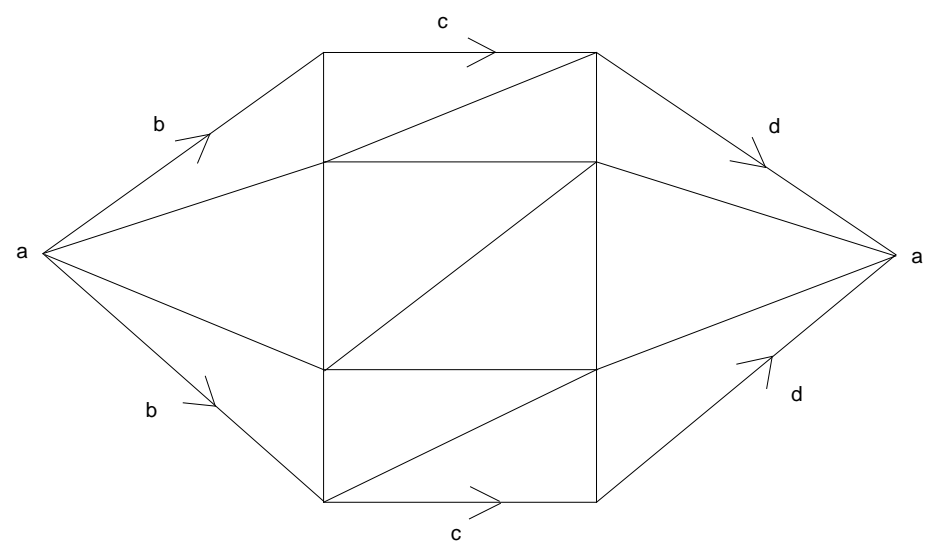

FIGURA 2.2: Identificação dos lados na construção do toro pinçado

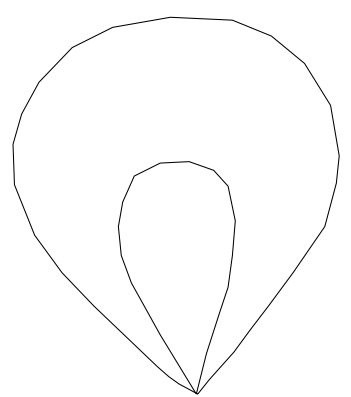

FIGURA 2.3: Toro pinçado

\subsubsection{Relação com os índices de um campo de vetores}

Teorema 2.11 (Poincaré-Hopf). Sejam $M$ uma variedade diferenciável compacta e $v$ um campo de vetores tangentes a $M$, com zeros isolados $a_{i}$. Então

$$
\chi(M)=\sum_{i} I\left(v, a_{i}\right)
$$


Demonstração. Daremos uma idéia da demonstração deste resultado. Inicialmente, constrói-se um campo de vetores (denominado campo de Hopf) cuja soma dos índices dá exatamente $\chi(M)$. O desenho a seguir o ilustra sobre os simplexos de dimensão 2 .

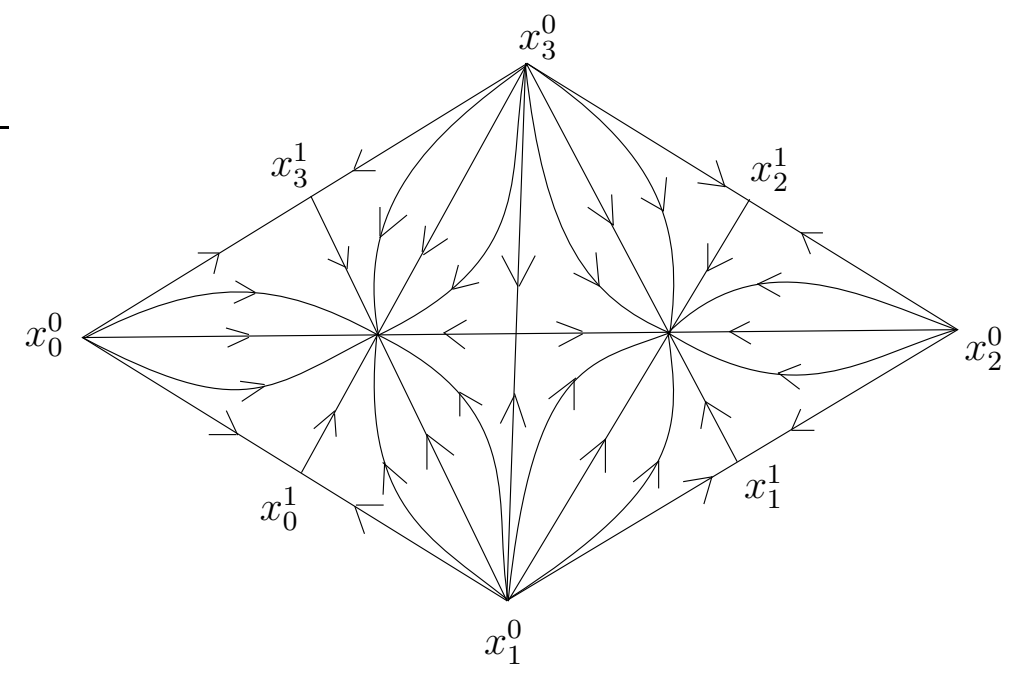

FIGURA 2.4: Campo de Hopf

Coloque $x_{\alpha}^{i}$ como sendo os baricentros dos simplexos de dimensão $i$. Este campo sai dos vértices $x_{\alpha}^{0}$ e, sobre cada segmento $\left[x_{\alpha}^{i}, x_{\beta}^{j}\right]$, com $i<j$, o campo é proporcional ao vetor $\left(x_{\alpha}^{i}, x_{\beta}^{j}\right)$ com coeficiente $\lambda \in[0,1]$ tal que $\lambda=0$ nos vértices e $\lambda=1$ no meio do segmento. Nos 2-simplexos, o campo é obtido a partir das coordenadas baricêntricas. A construção prossegue indutivamente.

Para finalizar a demonstração, é preciso mostrar que a soma dos índices das singularidades é sempre a mesma, independentemente do campo escolhido (vide [12] ou [16]).

Seguem dois exemplos de campos construídos na esfera e no toro. Note que, por este teorema, não é possível construir um campo de vetores tangentes a $S^{2}$ sem singularidades (é impossível pentear uma bola sem fazer redemoinho), ao contrário do toro: 

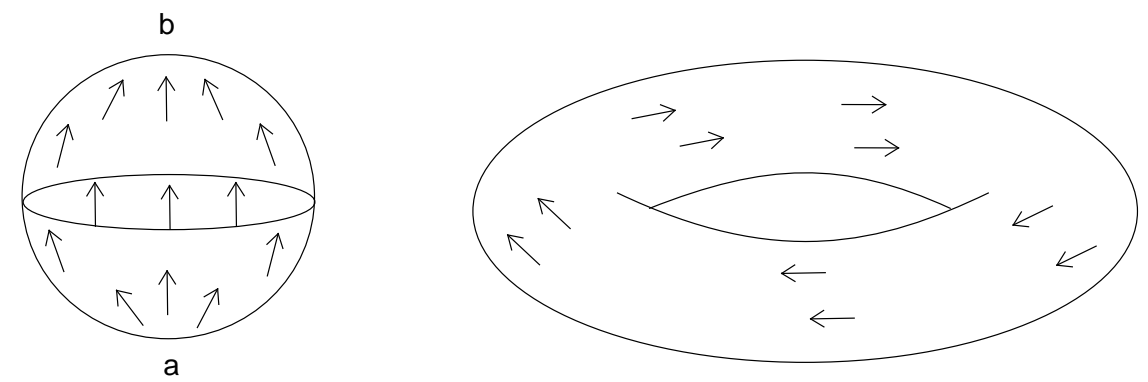

FIGURA 2.5: Campos na esfera e no toro 


\section{Capítulo 3}

\section{Classes de Stiefel-Whitney}

Neste capítulo, ainda com base em [18], daremos um resumo da teoria das classes características de Stiefel-Whitney para variedades suaves, classes essas em cohomologia, com grupo de coeficientes $\mathbf{Z}_{2}$. Serão enunciados os quatro axiomas, as suas propriedades básicas (assumiremos a existência e a unicidade das classes) e resultados que relacionam as classes com as variedades de Grassmann. Este capítulo tem caráter introdutório, servindo como modelo para o entendimento das seções seguintes.

\subsection{Classes características de Stiefel-Whitney}

Axioma 3.1. A cada $n$-fibrado vetorial $\xi$ de base $B$ está associada uma seqüência de classes em cohomologia $w_{j}(\xi) \in H^{j}(B)$, denominadas classes de Stiefel-Whitney de $\xi$. Pede-se que $w_{0}(\xi)=1 \in H^{0}(B)$, identidade do anel $H^{*}(B)$, e que $w_{i}(\xi)=0$, $i>n$.

Denominaremos a soma formal $w(\xi)=1+w_{1}(\xi)+\ldots+w_{n}(\xi) \in H^{*}(B)$ como classe total de Stiefel-Whitney (é um elemento invertivel deste anel).

Axioma 3.2 (Naturalidade). Se uma aplicação $f: B(\xi) \rightarrow B(\eta)$ pode ser levantada a uma aplicação entre fibrados $\xi \rightarrow \eta$, então $f^{*}\left(w_{i}(\eta)\right)=w_{i}(\xi)$.

Axioma 3.3 (Teorema do Produto de Whitney). Se $\xi$ e $\eta$ são fibrados de mesma base, então $w(\xi \oplus \eta)=w(\xi) \cdot w(\eta)$.

Axioma 3.4. Para o fibrado $\gamma_{1}^{1}$ sobre $P^{1}$, tem-se $w_{1}\left(\gamma_{1}^{1}\right) \neq 0$.

Proposição 3.5. Se $\xi$ é um fibrado que possui $k$ seções linearmente independentes então $w_{n-k+1}(\xi)=w_{n-k+2}(\xi)=\ldots=w_{n}(\xi)=0$. 
Quando nos referirmos ao fibrado tangente a uma variedade suave $M$, também utilizaremos a notação $w(M)$ para a classe do fibrado. A notação do elemento inverso da classe total $w(\xi)$ será $\bar{w}(\xi)=1+\bar{w}_{1}(\xi) \ldots+\bar{w}_{n}(\xi)+\ldots$

Teorema 3.6. A classe total de Stiefel-Whitney de $P^{n}$ é $w\left(P^{n}\right)=(1+a)^{n+1}$.

Uma demonstração análoga à prova deste teorema será feita no Teorema 5.13, no contexto das classes de Chern.

\subsection{Aplicação em imersões}

Quais espaços projetivos podem ser imersos em um espaço euclideano de uma dada dimensão?

Se $M$ é uma variedade em $\mathbb{R}^{n+k}$, o teorema do produto de Whitney, aliado a Proposição 1.12, diz que $w(\nu)=\bar{w}(M)$ (o que é conhecido como dualidade de Whitney). Daí, temos que $\bar{w}_{i}(M)=0$, se $i>k$.

Consideremos o caso do espaço projetivo $P^{9}$. Da proposição acima, $w\left(P^{9}\right)=$ $(1+a)^{10}=1+a^{2}+a^{8}$ (lembrando que estamos com coeficientes em $\mathbf{Z}_{2}$ ), assim, calculando o inverso, temos que $\bar{w}\left(P^{9}\right)=1+a^{2}+a^{4}+a^{6}$. Logo, se $P^{9}$ pudesse ser imerso em $\mathbb{R}^{9+k}$, então $k$ não poderia ser menor do que 6 . Usando o mesmo raciocínio para $n$ sendo potência de 2 , chegamos à conclusão de que $P^{n}$ não pode ser imerso em $\mathbb{R}^{2 n-2}$.

Whitney mostrou ([26]) que toda variedade suave compacta $M$, de dimensão $n>1$ pode ser imersa em $\mathbb{R}^{2 n-1}$. Ou seja, nosso cálculo acima dá a melhor estimativa possível quando $n$ é potência de 2 .

\subsection{Variedades de Grassmann}

Definição 3.7. A variedade de Grassmann $G_{n}\left(\mathbb{R}^{n+k}\right)$ é o conjunto dos $n$-planos em $\mathbb{R}^{n+k}$ passando pela origem.

A topologia para esta variedade é a seguinte. Um $n$-referencial em $\mathbb{R}^{n+k}$ é uma $n$-upla de vetores linearmente independentes. A coleção de todos os $n$-referenciais em $\mathbb{R}^{n+k}$ forma um aberto de $\mathbb{R}^{n+k} \times \ldots \times \mathbb{R}^{n+k}$ ( $n$ vezes) (pois seu complementar é claramente um subconjunto fechado). Este aberto é denominado variedade de Stiefel (com a topologia induzida) e denotado por $V_{n}\left(\mathbb{R}^{n+k}\right)$. A topologia da variedade de Grassmann então é a induzida pela aplicação 


$$
q: V_{n}\left(\mathbb{R}^{n+k}\right) \rightarrow G_{n}\left(\mathbb{R}^{n+k}\right)
$$

que associa, a cada $n$-upla de vetores, o espaço gerado por eles.

Com esta topologia, a variedade de Grassmann é uma variedade topológica (na verdade, um CW-complexo finito) compacta, de dimensão nk ([18], Lema 5.1). Além disso, temos que $G_{n}\left(\mathbb{R}^{n+k}\right)$ é homeomorfo a $G_{k}\left(\mathbb{R}^{n+k}\right)$. Observe que $P^{n}$ é a variedade de Grassmann $G_{1}\left(\mathbb{R}^{n+1}\right)$ (e, portanto, é homeomorfa a $G_{n}\left(\mathbb{R}^{n+1}\right)$ ).

Constrói-se também o $n$-fibrado $\gamma^{n}\left(\mathbb{R}^{n+k}\right)$ sobre a variedade de Grassmann $G_{n}\left(\mathbb{R}^{n+k}\right)$, consistindo dos pares $(X, v)$, com $X \in G_{n}\left(\mathbb{R}^{n+k}\right), v \in X$ e com projeção canônica.

Teorema 3.8 (Lema 5.3). Para qualquer $n$-fibrado $\xi$ de base compacta, existe uma aplicação entre fibrados $\xi \rightarrow \gamma^{n}\left(\mathbb{R}^{n+k}\right)$, para algum $k$ suficientemente grande.

Podemos tomar agora o conjunto de todos os $n$-subespaços em $\mathbb{R}^{\infty}$, obtendo a Grassmanniana $G_{n}$, com a topologia do limite direto (é um CW-complexo, logo é paracompacto - [19]). Analogamente ao caso finito, constrói-se o $n$-fibrado $\gamma^{n}$ sobre a Grassmanniana $G_{n}$, denominado fibrado universal.

Teorema 3.9 (Teorema 5.6). Para todo $n$-fibrado $\xi$ com base $B$ paracompacta, existe uma aplicação $f: B \rightarrow G_{n}$, que pode ser levantada a uma aplicação entre os fibrados $\xi$ e $\gamma^{n}$. Mais ainda, quaisquer duas aplicações com esta propriedade são homotópicas.

Teorema $\mathbf{3 . 1 0}$ (Teorema 7.1). (i) Não existem relações polinomiais entre $w_{1}\left(\gamma^{n}\right), \ldots, w_{n}\left(\gamma^{n}\right)$ (isto é, são algebricamente independentes);

(ii) O anel de cohomologia $H^{*}\left(G_{n}, \mathbf{Z}_{2}\right)$ é o anel dos polinômios com coeficientes em $\mathbf{Z}_{2}$, nas variáveis $w_{1}\left(\gamma^{n}\right), \ldots, w_{n}\left(\gamma^{n}\right)$. 


\section{Capítulo 4}

\section{Classe de Euler e aplicações}

Neste capítulo, construiremos uma importante classe em cohomologia, a classe de Euler (agora usando $\mathbf{Z}$ como grupo de coeficientes). Estudaremos sua relação com as classes de Stiefel-Whitney e a construção da seqüência de Gysin, que será usada posteriormente. Também apresentaremos aplicações, uma delas relacionada ao cálculo da característica de Euler-Poincaré. A referência utilizada é [18].

\subsection{Definição e propriedades básicas}

No capítulo 2, comentamos sobre orientações de variedades. Vamos definir orientações agora no contexto de homologia. Dado um $n$-espaço vetorial real $V$, denote por $V_{0}$ o conjunto $V-\{0\}$. Uma orientação para $V$ corresponde à escolha de um dos dois possíveis geradores do grupo de homologia singular $H_{n}\left(V, V_{0}, \mathbf{Z}\right) \simeq$ $H_{n}\left(\mathbb{R}^{n}, \mathbb{R}^{n}-\{0\}, \mathbf{Z}\right)$, que é cíclico infinito.

De fato, seja $\Delta_{n}$ o $n$-simplexo padrão, com vértices ordenados canonicamente. Considere um mergulho linear $\sigma: \Delta_{n} \rightarrow V$ que preserve orientação, levando o baricentro de $\Delta_{n}$ em 0 ( $\sigma$ está em $C_{n}(V)$, mas não em $\left.C_{n}\left(V_{0}\right)\right)$. Assim, o bordo de $\Delta_{n}$ é levado em $V_{0}\left(\partial \sigma \in C_{n-1}\left(V_{0}\right)\right)$. Temos então que $\sigma$ é representante de um elemento $\bar{\sigma}$ não nulo de $Z_{n}\left(V, V_{0}, \mathbf{Z}\right) \subset \frac{C_{n}(V)}{C_{n}\left(V_{0}\right)}$. Além disso, a classe de $\bar{\sigma}$, denotada por $\mu_{V}$, é um gerador de $H_{n}\left(V, V_{0}, \mathbf{Z}\right)$. Em cohomologia, $H^{n}\left(V, V_{0}, \mathbf{Z}\right)$ tem um gerador $u_{V}$ definido pela avaliação: $\left\langle u_{V}, \mu_{V}\right\rangle=1$.

Definiremos agora uma orientação para um $n$-fibrado vetorial real com base $B$ e projeção $\pi: E \rightarrow B$. Denote por $E_{0}$ o conjunto dos elementos não nulos do espaço total $E$ do fibrado.

Definição 4.1. Uma orientação (em cohomologia) para um fibrado $\xi$ é uma 
escolha de geradores $u_{F} \in H^{n}\left(F, F_{0}, \mathbf{Z}\right)$ para cada fibra (n-espaço vetorial) $F$ de $\xi$ satisfazendo a condição de que todo ponto $b \in B$ admita uma vizinhança $N$ e uma classe $u \in H^{n}\left(\pi^{-1} N,\left(\pi^{-1} N\right)_{0}, \mathbf{Z}\right)$ tal que a restrição $u_{\mid\left(F, F_{0}\right)}$ vale $u_{F}$.

Quando não houver dúvida sobre o grupo de coeficientes, este será omitido. Suponha agora que o fibrado $\xi$ é orientável:

Teorema 4.2 (Teorema 9.1). Para $i<n$, temos que $H^{i}\left(E, E_{0}\right)=0$. Além disso, existe uma única classe (denominada classe fundamental) $u \in H^{n}\left(E, E_{0}\right)$ tal que, para toda fibra $F, u_{\mid\left(F, F_{0}\right)}=u_{F}$ e a correspondência $y \rightarrow y \cup u$ é um isomorfismo entre $H^{k}(E)$ e $H^{k+n}\left(E, E_{0}\right), k \in \mathbf{Z}$.

Definição 4.3. Definimos o isomorfismo de Thom como sendo a seguinte aplicação

$$
\begin{gathered}
\phi: H^{k}(B) \rightarrow H^{k+n}\left(E, E_{0}\right) \\
x \mapsto \pi^{*} x \cup u .
\end{gathered}
$$

Definição 4.4. A classe de Euler então é definida como o elemento $e(\xi) \in H^{n}(B)$ tal que $\phi(e(\xi))=u_{\mid E} \cup u$.

Proposição 4.5 (Propriedades básicas da classe de Euler). (i) (Naturalidade) Se $f: B \rightarrow B^{\prime}$ pode ser levantada a uma aplicação entre fibrados $f: \xi \rightarrow \xi^{\prime}$, então $f^{*}\left(e\left(\xi^{\prime}\right)\right)=e(\xi)$;

(ii) (Ordem) Se n é ímpar, então e( $\xi)$ tem ordem 2, isto é, e $(\xi)+e(\xi)=0$;

(iii) (Relação com as classes de Stiefel-Whitney) O homomorfismo $h: H^{n}(B, \mathbf{Z}) \rightarrow$ $H^{n}\left(B, \mathbf{Z}_{2}\right)$ que reduz os coeficientes módulo 2 é tal que $h(e(\xi))=w_{n}(\xi)$;

(iv) (Soma de Whitney) $e\left(\xi \oplus \xi^{\prime}\right)=e(\xi) \cup e\left(\xi^{\prime}\right)$.

Todas as propriedades acima são de fácil demonstração.

Proposição 4.6. Se o fibrado orientável $\xi$ possui seção não nula, então e $(\xi)=0$.

Demonstração. Suponha que $s: B \rightarrow E_{0}$ é uma seção não nula. A composição (identidade):

$$
B \stackrel{s}{\longrightarrow} E_{0} \stackrel{i n c l}{\longrightarrow} E \stackrel{\pi}{\longrightarrow} B
$$

dá origem em cohomologia a: 


$$
H^{n}(B) \stackrel{\pi^{*}}{\longrightarrow} H^{n}(E) \stackrel{(\text { incl })^{*}}{\longrightarrow} H^{n}\left(E_{0}\right) \stackrel{s^{*}}{\longrightarrow} H^{n}(B)
$$

Pela construção da classe de Euler, temos $\pi^{*} e(\xi)=u_{\mid E}$. Daí, $(\text { incl })^{*}\left(\pi^{*} e(\xi)\right)=(\text { incl })^{*} u_{\mid E}$. Mas olhando para a seqüência exata em cohomologia

$$
\cdots \longrightarrow H^{n}\left(E, E_{0}\right) \longrightarrow H^{n}(E) \stackrel{(i n c l)^{*}}{\longrightarrow} H^{n}\left(E_{0}\right) \longrightarrow \cdots
$$

temos que $u_{\mid E} \in \operatorname{Ker}(\text { incl })^{*}$ e então, $(\text { incl })^{*}\left(\pi^{*} e(\xi)\right)=0$. Do comentário inicial da demonstração, segue que $e(\xi)=s^{*}\left((\text { incl })^{*}\left(\pi^{*} e(\xi)\right)\right)=0$.

\subsection{Seqüência de Gysin de um fibrado vetorial}

Seja $\xi$ um $n$-fibrado vetorial com projeção $\pi: E \rightarrow B$. Restringindo $\pi$ a $E_{0}$, obtemos uma aplicação $\pi_{0}: E_{0} \rightarrow B$.

Considere a seqüência exata em cohomologia, do Axioma 1.21, com qualquer grupo de coeficientes $\Lambda$ :

$$
\cdots \longrightarrow H^{j}\left(E, E_{0}\right) \longrightarrow H^{j}(E) \longrightarrow H^{j}\left(E_{0}\right) \longrightarrow H^{j+1}\left(E, E_{0}\right) \longrightarrow \cdots
$$

Usando o isomorfismo de Thom $\cup u: H^{j-n}(B) \rightarrow H^{j}\left(E, E_{0}\right)$ e o isomorfismo entre $H^{*}(E)$ e $H^{*}(B)$ a seqüência exata se torna:

$$
\cdots \longrightarrow H^{j-n}(B) \stackrel{\cup e(\xi)}{\longrightarrow} H^{j}(B) \stackrel{\pi_{0}^{*}}{\longrightarrow} H^{j}\left(E_{0}\right) \longrightarrow H^{j-n+1}(B) \longrightarrow \cdots
$$

Esta nova seqüência exata é denominada seqüência de Gysin.

Como caso particular importante desta seqüência, considere $\tilde{B}$ um recobrimento duplo de $B$. Assim, temos uma aplicação $r: \tilde{B} \rightarrow B$ onde cada ponto de $B$ tem uma vizinhança $U$ cuja imagem inversa por $r$ é formada de duas cópias disjuntas de $U$.

Construiremos um novo fibrado sobre $B$. Seu espaço total $E$ é obtido identificando os pontos $(x, t)$ e $\left(x^{\prime},-t\right)$ de $\tilde{B} \times \mathbb{R}$ tais que $r(x)=r\left(x^{\prime}\right)$. Desta forma, temos $\tilde{B}$ como retrato de deformação de $E_{0}$. A seqüência de Gysin então se torna (com coeficientes em $\left.\mathbf{Z}_{2}\right)$ :

$$
\cdots \longrightarrow H^{j-1}(B) \stackrel{\cup w_{1}(\xi)}{\longrightarrow} H^{j}(B) \longrightarrow H^{j}(\tilde{B}) \longrightarrow H^{j}(B) \longrightarrow \cdots
$$




\subsubsection{O fibrado universal orientável}

Denotaremos aqui $\tilde{G}_{n}\left(\mathbb{R}^{n+k}\right)$ a variedade de Grassmann consistindo de todos os $n$-planos orientados em $\mathbb{R}^{n+k}$, que é um recobrimento duplo de $G_{n}\left(\mathbb{R}^{n+k}\right)$. A topologia dada ainda é a induzida da variedade de Stiefel $V_{n}\left(\mathbb{R}^{n+k}\right)$ (3.1). Fazendo o limite direto $(k \rightarrow \infty)$, obtemos o CW-complexo $\tilde{G}_{n}$.

O fibrado universal $\gamma^{n}$ pode ser levantado a um $n$-fibrado $\tilde{\gamma}^{n}$ sobre $\tilde{G}_{n}$. Assim, para todo fibrado orientável $\xi$ existe uma aplicação entre fibrados $f: \xi \rightarrow \tilde{\gamma}^{n}$ que preserva orientação. Assim como feito para $G_{n}$, daremos uma descrição para o anel de cohomologia $H^{*}\left(\tilde{G}_{n}, \mathbf{Z}_{2}\right)$.

Teorema 4.7. O anel $H^{*}\left(\tilde{G}_{n}, \mathbf{Z}_{2}\right)$ é o anel de polinômios nas classes $w_{2}\left(\tilde{\gamma}^{n}\right), \ldots, w_{n}\left(\tilde{\gamma}^{n}\right)$ com coeficientes em $\mathbf{Z}_{2}$.

Demonstração. Aplicando a seqüência exata obtida em (4.2), temos:

$$
\cdots \longrightarrow H^{j-1}\left(G_{n}\right) \stackrel{\cup w_{1}(\xi)}{\longrightarrow} H^{j}\left(G_{n}\right) \stackrel{r^{*}}{\longrightarrow} H^{j}\left(\tilde{G}_{n}\right) \longrightarrow H^{j}\left(G_{n}\right) \longrightarrow \cdots
$$

Temos que $w_{1}(\xi) \in H^{1}\left(G_{n}, \mathbf{Z}_{2}\right)=\left\{0, w_{1}(\xi)\right\}$ (pelo Teorema 3.10), onde $\xi$ é o 1-fibrado associado ao recobrimento duplo de $G_{n}$ e $r: \tilde{G}_{n} \rightarrow G_{n}$ é a aplicação de recobrimento.

Se tivéssemos $w_{1}(\xi)=0$, então teríamos uma seqüência cindida:

$$
0 \longrightarrow H^{0}\left(G_{n}\right) \longrightarrow H^{0}\left(\tilde{G}_{n}\right) \longrightarrow H^{0}\left(G_{n}\right) \longrightarrow 0
$$

e daí $H^{0}\left(\tilde{G}_{n}\right)=\mathbf{Z}_{2} \times \mathbf{Z}_{2}$, o que diz que $\tilde{G}_{n}$ tem duas componentes conexas, uma contradição. Logo, $w_{1}(\xi)=w_{1}\left(\gamma^{n}\right)$.

Assim, temos

$$
\cdots \longrightarrow H^{0}\left(G_{n}\right) \stackrel{\cup w_{1}\left(\gamma^{n}\right)}{\longrightarrow} H^{1}\left(G_{n}\right) \stackrel{r^{*}}{\longrightarrow} H^{1}\left(\tilde{G}_{n}\right) \longrightarrow \cdots
$$

e como a seqüência é exata, $H^{1}\left(G_{n}\right)=\left\{0, w_{1}\left(\gamma^{n}\right)\right\}=\operatorname{Im}\left(\cup w_{1}\left(\gamma^{n}\right)\right)=\operatorname{Ker}\left(r^{*}\right)$ e daí $0=r^{*}\left(w_{1}\left(\gamma^{n}\right)\right)=w_{1}\left(\tilde{\gamma}^{n}\right)$ (Note que isto implica que, para todo fibrado orientável $\eta$ de base paracompacta, $\left.w_{1}(\eta)=0\right)$

Agora, observando a seqüência de Gysin (4.3), se mostrarmos que $r^{*}$ é sobrejetora, o resultado fica provado.

Para isso, note que

$$
\frac{H^{j}\left(\tilde{G}_{n}\right)}{\operatorname{Im}\left(r^{*}\right)} \simeq \operatorname{Ker}\left(\cup w_{1}\left(\gamma^{n}\right)\right)=\left\{w \in H^{j}\left(G_{n}\right): w \cup w_{1}\left(\gamma^{n}\right)=0\right\}
$$


Mas este último conjunto consiste apenas do elemento neutro pois, caso contrário teríamos uma relação polinomial com elementos de $H^{*}\left(G^{n}\right)$, o que não é possível (novamente, usando o Teorema 3.10). Portanto, $H^{j}\left(\tilde{G}_{n}\right) \simeq \operatorname{Im}\left(r^{*}\right)$ e daí $r^{*}$ é sôbre em todos os níveis de cohomologia. Além disso, têm-se que $w_{j}\left(\tilde{\gamma}^{n}\right) \neq 0$, $j=2, \ldots, n$, e que $H^{1}\left(\tilde{G}_{n}\right)=\{0\}$.

\subsection{Os fibrados normal e tangente}

Seja $M$ uma variedade suave, compacta, de dimensão $n$, mergulhada numa variedade riemanniana suave $A$, de dimensão $n+k$. Iremos estudar as classes do fibrado normal $\nu$ de $M$ em $A$.

Teorema 4.8 (Teorema 11.1, Vizinhança Tubular). Existe uma vizinhança de $M$ em A localmente difeomorfa ao espaço total $E$ do fibrado normal, tal que o difeomorfismo leva $x \in M$ no vetor nulo da fibra de $x$.

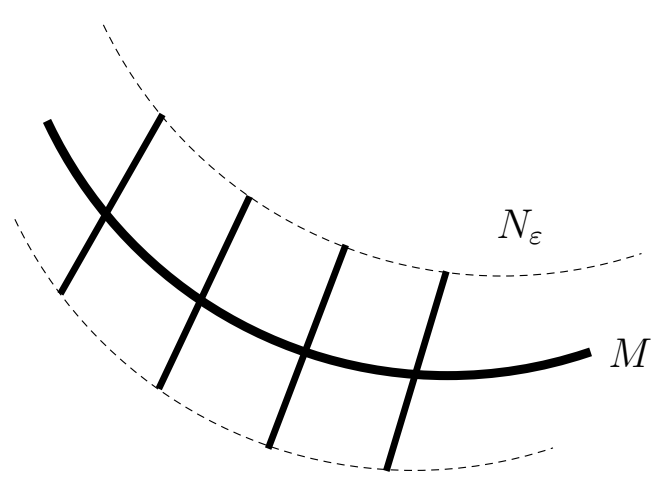

FIGURA 4.1: Vizinhança tubular de $M$

Denotamos por $E(\varepsilon)=\{(x, v) \in E:|v|<\varepsilon\}$ a vizinhança da imagem de $M$ (no fibrado). Mais geralmente, quando $M$ não é compacta, $\varepsilon$ pode ser tomado como uma função real positiva e suave.

Definimos também a aplicação exponencial $\operatorname{Exp}: E(\varepsilon) \rightarrow A$, que a cada ponto $(x, v) \in E(\varepsilon)$ associa o ponto final $\gamma(1)$ da curva geodésica $\gamma:[0,1] \rightarrow A$ de ponto inicial $\gamma(0)=x$, de comprimento $|v|$ e de velocidade inicial $\gamma^{\prime}(0)=v$.

Os teoremas de Equações Diferenciais garantem a existência e suavidade da aplicação exponencial em $E(\varepsilon)$, para $\varepsilon$ suficientemente pequeno.

Pelo teorema da função implícita, aplicado no ponto $(x, 0)$, obtemos uma vizinhança do mesmo em $E(\varepsilon)$ difeomorfa a um aberto de $A$. Mais ainda, se $\varepsilon$ é 
pequeno, $E(\varepsilon)$ é levado num aberto $N_{\varepsilon} \subset A$ (vizinhança tubular) pela aplicação exponencial. Assim, temos:

Corolário 4.9 (Corolário 11.2). Se $M$ é fechado em $A$, então o anel $H^{*}\left(E, E_{0}, \Lambda\right)$ é isomorfo a $H^{*}(A, A-M, \Lambda)$.

Considere agora a classe fundamental $u \in H^{k}\left(E, E_{0}, \mathbf{Z}_{2}\right)$. A ela corresponde a classe $u^{\prime} \in H^{k}\left(A, A-M, \mathbf{Z}_{2}\right)$. Se o fibrado normal for orientável, considera-se $\mathbf{Z}$ como grupo de coeficientes.

Teorema 4.10 (Teorema 11.3.). Se M é fechado em A, então a seguinte composição de restrições

$$
H^{k}(A, A-M) \longrightarrow H^{k}(A) \longrightarrow H^{k}(M)
$$

leva a classe $u^{\prime}$ em $w_{k}\left(\nu^{k}\right)$ (ou em e $\left.\left(\nu^{k}\right)\right)$.

Definição 4.11. O elemento $u_{\mid A}^{\prime}$, imagem de $u^{\prime}$ em $H^{k}(A)$, é a classe de cohomologia dual de $M$, em codimensão $k$.

Segue que, se $u_{\mid A}^{\prime}=0$, então $w_{k}\left(\nu^{k}\right)=0$ (ou $\left.e\left(\nu^{k}\right)=0\right)$. Assim, se $M$ pode ser mergulhada em $\mathbb{R}^{n+k}$, então $w_{k}\left(\nu^{k}\right)=0\left(\right.$ ou $\left.e\left(\nu^{k}\right)=0\right)$.

Sabemos da dualidade de Whitney que $w_{k}\left(\nu^{k}\right)=\overline{w_{k}}(M)$.

Logo, se $\overline{w_{k}}(M) \neq 0$, temos que $M$ não pode ser mergulhada em $\mathbb{R}^{n+k}$.

Por exemplo, se $n=2^{s}, \overline{w_{n-1}}\left(P^{n}\right)=a^{n-1} \neq 0$ e então $P^{n}$ não pode ser mergulhado em $\mathbb{R}^{2 n-1}$.

Sabemos que Whitney também provou ([27]) que toda variedade suave com base enumerável pode ser suavemente mergulhada em $\mathbb{R}^{2 n}$ (como subconjunto fechado).

Seja agora $M$ uma variedade riemanniana. O produto $M \times M$ também tem estrutura de variedade riemanniana induzida canonicamente pela estrutura de $M$. Através da aplicação diagonal, pode-se mergulhar suavemente $M$ (como subconjunto fechado) em $M \times M$. Desta forma, o fibrado $\nu^{n}$ normal a $M$ em $M \times M$ é isomorfo a ao fibrado tangente de $M$.

De fato, se $x \in M$, um vetor $(a, b) \in T_{x} M \times T_{x} M \simeq T_{x \times x}(M \times M)$ é tangente a $M$ se, e somente se, $a=b$. Como $(u, v) \cdot(a, a)=0$, para todo $a \in T_{x} M$ se, e somente se, $u=-v$, podemos então definir a correspondência $(x, v) \longmapsto((x, x),(-v, v))$ entre os espaços totais dos fibrados desejados. 
Sempre assumindo que $M$ é uma variedade orientável, ou que $\Lambda=\mathbf{Z}_{2}$, concluímos que $u_{\mid \Delta(M)}^{\prime}=e\left(\nu^{n}\right)=e\left(\tau_{M}\right)$. Uma nova caracterização de $u^{\prime}$ é dada a seguir.

Considere um elemento $x \in M$ fixado, e $u_{x}$ o gerador do grupo $H^{n}(M, M-$ $\{x\})$ tal que $<u_{x}, \mu_{x}>=1\left(\right.$ ou $u_{x} \neq 0$ no caso de $\Lambda=\mathbf{Z}_{2}$ ). Defina a seguinte aplicação:

$$
\begin{gathered}
j_{x}:(M, M-\{x\}) \rightarrow(M \times M, M \times M-\Delta(M)) \\
j_{x}(y) \mapsto(x, y) .
\end{gathered}
$$

Temos então que a classe $u^{\prime}$ é a única com a seguinte propriedade:

Proposição 4.12. $j_{x}^{*}\left(u^{\prime}\right)=u_{x}$.

Demonstração. Seja $N$ uma vizinhança de 0 suficientemente pequena em $D M_{x}$. Defina a aplicação:

$$
\begin{gathered}
e:(N, N-\{0\}) \rightarrow(M \times M, M \times M-\Delta(M)) \\
v \mapsto(\operatorname{Exp}(x,-v), \operatorname{Exp}(x, v)) .
\end{gathered}
$$

Temos que a aplicação induzida em cohomologia $e^{*}: H^{n}(M \times M, M \times M-$ $\Delta(M)) \simeq H^{n}\left(E, E_{0}\right) \rightarrow H^{n}(N, N-\{0\})$ é tal que $e^{*}\left(u^{\prime}\right)$ é o gerador escolhido de $H^{n}(N, N-\{0\})$.

Agora considere a homotopia $(x, t) \mapsto(\operatorname{Exp}(x,-t v), \operatorname{Exp}(x, v)), t \in[0,1]$ entre $e$ e $j_{x} \circ \operatorname{Exp}$.

Temos então que $\left(j_{x} \circ \operatorname{Exp}\right)^{*}\left(u^{\prime}\right)$ também é o gerador escolhido de $H^{n}(N, N-$ $\{0\}) \simeq H^{n}(M, M-\{x\}$ ) (sendo o isomorfismo dado pela aplicação Exp), o que conclui a demonstração.

Definição 4.13. A classe dual $u^{\prime \prime} \in H^{n}(M \times M)$, obtida de $u^{\prime}$ pelo homomorfismo restrição, é denominada classe de cohomologia diagonal.

\subsection{Aplicações}

Nosso objetivo nesta seção é caracterizar a classe diagonal e utilizá-la para relacionar a classe de Euler e a característica de Euler-Poincaré. 
Lema 4.14 (Lema 11.8). Para todo elemento $a \in H^{*}(M)$, temos que $(a \times 1) \cup u^{\prime \prime}=$ $(1 \times a) \cup u^{\prime \prime}$.

A seguir, definiremos uma nova operação: o produto Slant: $H^{*}(X \times Y) \times$ $H_{*}(Y) \rightarrow H^{*}(X)$, com coeficientes em $\Lambda$. Aqui consideraremos o caso em que $\Lambda$ é corpo e $X, Y$ são complexos finitos (para o caso geral, vide a referência do capítulo).

Nessas condições, vale a fórmula de Künneth: $H^{*}(X \times Y) \simeq H^{*}(X) \times H^{*}(Y)$.

Daí, para $p \in H^{*}(X \times Y)$, e $\beta \in H_{*}(Y)$, definimos o produto Slant $p_{\mid \beta}$ da seguinte forma: pela fórmula de Künneth, escreva $p=a \times b$, com $a \in H^{*}(X)$ e $b \in H^{*}(Y)$ e coloque $p_{\mid \beta}=<b, \beta>\cdot a$.

Segue desta definição a seguinte propriedade, para $c \in H^{*}(X)$ :

$$
((c \times 1) \cup p)_{\mid \beta}=c \cup\left(p_{\mid \beta}\right) .
$$

De fato, pela Proposição 1.28 e propriedades do produto cup, temos que

$$
\begin{aligned}
((c \times 1) \cup p)_{\mid \beta}=((c \cup a) \times(1 \cup b))_{\mid \beta} & = \\
<b, \beta>\cdot(c \cup a) & = \\
c \cup(<b, \beta>a) & =c \cup\left(p_{\mid \beta}\right) .
\end{aligned}
$$

Lema 4.15. Suponha que $M$ é uma variedade compacta (de tal forma que a classe fundamental de homologia $\mu \in H_{n}(M)$ está definida). Então a classe diagonal $u^{\prime \prime}$ satisfaz $\left(u^{\prime \prime}\right)_{\mid \mu}=1$.

Demonstração. Para cada $x \in M$, calculemos a imagem de $u_{\mid \mu}^{\prime \prime}$ na restrição a $H^{0}(x) \simeq \Lambda$ e mostremos que a mesma vale 1 .

Construimos o seguinte diagrama comutativo:

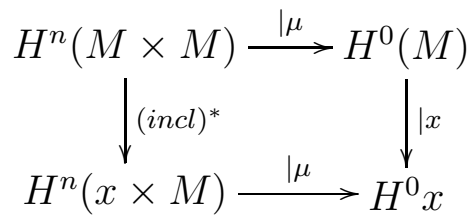

Note que $(i n c l)^{*}=\left(i_{x} \circ p_{2}\right)^{*}$ onde $i_{x}: M \rightarrow x \times M$ é tal que $i_{x}(y)=(x, y)$. Daí, temos $(\text { incl })^{*} u^{\prime \prime}=1 \cup(\text { incl })^{*} u^{\prime \prime}=p_{1}^{*}(1) \cup p_{2}^{*}\left(i_{x}^{*} u^{\prime \prime}\right)=1 \times i_{x}^{*} u^{\prime \prime}$, usando (1.2).

Aplicando o produto Slant, segue que $\left(1 \times i_{x}^{*} u^{\prime \prime}\right)_{\mid \mu}=<i_{x}^{*} u^{\prime \prime}, \mu>$. Mas como o diagrama é comutativo, $\left(u_{\mid \mu}^{\prime \prime}\right)_{\mid x}=\left(1 \times i_{x}^{*} u^{\prime \prime}\right)_{\mid \mu}=<i_{x}^{*} u^{\prime \prime}, \mu>$. Resta então mostrar que $<i_{x}^{*} u^{\prime \prime}, \mu>=1$. Note que a aplicação $i_{x}^{*}$ é semelhante a $j_{x}^{*}$ da Proposição 4.12, $\operatorname{logo}<i_{x}^{*} u^{\prime \prime}, \mu>=<\left(j_{x}^{*} u^{\prime}\right)_{\mid M}, \mu>$. Ou seja, pelo mesmo teorema, basta mostrar que 
$\beta \doteq<\left(j_{x}^{*} u^{\prime}\right)_{\mid M}, \mu>$ é igual a $\alpha \doteq<j_{x}^{*}\left(u^{\prime}\right), \mu_{x}>$, já que este vale 1 . Isto segue ao observarmos o seguinte diagrama

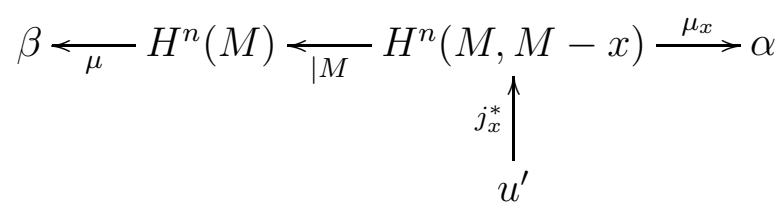

e do fato de que $\mid M$ é a aplicação dual da aplicação em homologia que leva $\mu \mathrm{em}$ $\mu_{x}$.

Teorema 4.16 (Dualidade de Poincaré). A toda base $b_{1}, \ldots, b_{r}$ de $H^{*}(M)$ corresponde uma base dual $b_{1}^{\prime}, \ldots, b_{r}^{\prime}$ satisfazendo $\left\langle b_{i} \cup b_{j}^{\prime}, \mu\right\rangle=\delta_{i j}$.

Demonstração. Pela fórmula de Künneth, podemos escrever $u^{\prime \prime} \in H^{n}(M \times M)$ como $u^{\prime \prime}=\sum_{i=1}^{r} b_{i} \times c_{i}$, onde $\operatorname{dim}\left(b_{i}\right)+\operatorname{dim}\left(c_{i}\right)=n$ e cada $c_{i}$ é bem determinado.

Aplicando o produto Slant no primeiro membro da igualdade do Lema 4.14, temos, para $a \in H^{*}(M)$ :

$$
\left((a \times 1) \cup u^{\prime \prime}\right)_{\mid \mu}=a \cup\left(u^{\prime \prime}\right)_{\mid \mu}=a .
$$

onde aplicamos a equação (4.4), e o Lema 4.15 em seguida.

Agora olharemos para o segundo membro da igualdade do Lema 4.13.

Como

$$
\begin{aligned}
(1 \times a) \cup u^{\prime \prime}= & (1 \times a) \cup \sum\left(b_{i} \times c_{i}\right)= \\
& \sum(1 \times a) \cup\left(b_{i} \times c_{i}\right)=\sum(-1)^{\operatorname{dim}(a) \cdot \operatorname{dim}\left(b_{i}\right)} b_{i} \times\left(a \cup c_{i}\right),
\end{aligned}
$$

aplicando o produto Slant, segue que:

$$
\left((1 \times a) \cup u^{\prime \prime}\right)_{\mid \mu}=\sum(-1)^{\operatorname{dim}(a) \cdot \operatorname{dim}\left(b_{i}\right)}<a \cup c_{i}, \mu>b_{i} .
$$

Portanto, igualando (4.5) e (4.6),

$$
a=\sum(-1)^{\operatorname{dim}(a) \cdot \operatorname{dim}\left(b_{i}\right)}<a \cup c_{i}, \mu>b_{i} .
$$

Como a é genérico, substituindo por $b_{j}$, temos que

$$
(-1)^{\operatorname{dim}\left(b_{j}\right) \cdot \operatorname{dim}\left(b_{i}\right)}<b_{j} \cup c_{i}, \mu>=\delta_{i j} .
$$

Tomando então $b_{i}^{\prime}=(-1)^{\operatorname{dim}\left(b_{i}\right)} c_{i}$, segue a propriedade desejada.

Fixado $k$, se $b_{i} \in H^{k}(M)$, os elementos $b_{i}^{\prime}$ de $H^{n-k}(M)$ serão linearmente independentes. De fato, tomando uma combinação linear nula dos mesmos, $\sum \alpha_{i} b_{i}^{\prime}=$ 
0 , teríamos, para todo $b_{t} \in H^{k}(M)$, que $0=<\sum \alpha_{i} b_{i}^{\prime} \cup b_{t}, \mu>=\sum \alpha_{i}<b_{i}^{\prime} \cup b_{t}, \mu>=$ $\alpha_{t}<b_{t}^{\prime} \cup b_{t}, \mu>=\alpha_{t}$.

Além disso, existe um isomorfismo entre $H^{k}(M)$ e o dualizado de $H^{n-k}(M)$ pelo funtor Hom. Ele associa a cada elemento $a \in H^{k}(M)$ a aplicação $h_{a}$ tal que $h_{a}(b)=\left\langle a \cup b, \mu>\right.$, para todo $b \in H^{n-k}(M)$. De fato, vê-se facilmente que a mesma é linear. O argumento da injetividade é similar ao que mostra a independência linear acima. Assim, realmente temos que $b_{1}^{\prime}, \ldots, b_{r}^{\prime}$ formam uma base de $H^{*}(M)$

Segue deste teorema a útil representação para a classe diagonal:

$$
u^{\prime \prime}=\sum_{i=1}^{r}(-1)^{\operatorname{dim}\left(b_{i}\right)} b_{i} \times b_{i}^{\prime} .
$$

O teorema da dualidade de Poincaré para variedades compactas é comumente enunciado na literatura como isomorfismo entre $H_{n-k}(M)$ e $H^{k}(M)$.

Esta versão pode ser facilmente provada a partir do teorema anterior por meio do produto Slant. A cada classe $\beta \in H_{n-k}(M)$ associe o produto $\left(u^{\prime \prime}\right)_{\mid \beta}=$ $\sum(-1)^{\operatorname{dim}\left(b_{i}\right)}\left(b_{i} \times b_{i}^{\prime}\right)_{\mid \beta}$.

Assim, se existisse um elemento $\beta$ tal que $\left(u^{\prime \prime}\right)_{\mid \beta}=0$, então, para todo $b_{i}^{\prime} \in$ $H^{n-k}(M)$, teríamos $<b_{i}^{\prime}, \beta>=0$, donde $\beta=0$, e a aplicação realmente definirá um isomorfismo.

Finalmente, através da representação acima de $u^{\prime \prime}$, temos o seguinte corolário, objetivo da seção:

Corolário 4.17. Se $M$ é uma variedade suave, compacta e orientável, então

$$
<e(M), \mu>=\chi(M)
$$

(usando coeficientes racionais).

Demonstração. Lembremos que, sendo $\Delta: M \rightarrow M \times M$ a aplicação diagonal, pelo Teorema 4.10, $e(M)=\Delta^{*}\left(u^{\prime \prime}\right)$.

Usando a caracterização de $u^{\prime \prime}$ em (4.7) e as propriedades do produto cup, segue que

$$
e(M)=\sum(-1)^{\operatorname{dim}\left(b_{i}\right)} b_{i} \cup b_{i}^{\prime}
$$


Assim,

$$
<e(M), \mu>=\sum(-1)^{\operatorname{dim}\left(b_{i}\right)}=\sum(-1)^{k} \operatorname{dim}\left(H^{k}(M)\right)=\chi(M) .
$$

Observação 4.18. Para $n$ ímpar, podemos dar uma outra prova deste fato. Como $\operatorname{dim}\left(H^{k}(M)\right)=\operatorname{dim}\left(H^{n-k}(M)\right)$, então $\chi(M)=0$. Além disso, a classe de Euler neste caso também será nula, pois é um elemento de ordem 2 de $H^{n}(M)$, pela Proposição 4.5. 


\section{Capítulo 5}

\section{Classes de Chern}

\subsection{Variedades e fibrados complexos}

Fibrados complexos são introduzidos de forma análoga aos reais. A diferença básica é que agora cada fibra é um espaço vetorial complexo. A referência para este capítulo é [18].

Definição 5.1. Um fibrado complexo $\omega$ de dimensão $n$ sobre um espaço topológico $B$ consiste de um espaço topológico $E$ e de uma projeção $\pi: E \rightarrow B$ tal que todo ponto $b \in B$ admite uma vizinhança $U$ e um homeomorfismo $h: \pi^{-1}(U) \rightarrow U \times \mathbb{C}^{n}$. Além disso, para todo $b \in B, \pi^{-1}(b)$ é um espaço vetorial complexo, e a restrição de $h$ a $\pi^{-1}(b)$ é um isomorfismo sobre $b \times \mathbb{C}^{n}$.

Assim como é feito no caso real, podemos construir fibrados com somas de Whitney e fibrados induzidos.

É claro que um $n$-fibrado complexo $\omega$ pode ser visto como um $2 n$-fibrado real (este será denotado por $\omega_{R}$ ). Agora, um $n$-fibrado complexo pode ser construído a partir de um $2 n$-fibrado real se o mesmo admitir uma estrutura complexa:

Definição 5.2. Uma estrutura complexa num $2 n$-fibrado $\xi$ é uma aplicação $J$ : $E(\xi) \rightarrow E(\xi)$ que é $\mathbb{R}$-linear em cada fibra e tal que $J(J(v))=-v$, para todo $v \in E(\xi)$.

Desta forma, cada fibra $F_{b}(\xi)$ se torna um espaço vetorial complexo.

Seja agora $U$ um aberto em $\mathbb{C}^{n}$. Temos que o espaço total do fibrado tangente, $E\left(\tau_{U}\right)=U \times \mathbb{C}^{n}$ tem estrutura complexa $J_{0}(u, v)=(u, i v)$. Se $M$ é uma variedade suave de dimensão $2 n$, uma estrutura complexa em $\tau_{M}$ é chamada uma estrutura quase complexa em $M$. 
Definição 5.3. Uma estrutura complexa em $M$ é uma estrutura complexa $J$ em $\tau_{M}$ que satisfaz a seguinte propriedade: todo ponto de $M$ possui uma vizinhança difeomorfa a um aberto de $\mathbb{C}^{n}$ por um difeomorfismo $h$ tal que $d h \circ J=J_{0} \circ d h$ (isto é, $h$ é holomorfa, ou analítica).

O seguinte resultado será importante nas próximas seções:

Proposição 5.4. Se $\omega$ é um n-fibrado vetorial complexo, então $\omega_{R}$ admite uma orientação.

A demonstração é bem simples. Basta encontrar a matriz mudança de base entre duas bases de fibras de $\omega_{R}$ e mostrar que seu determinante é positivo.

Dada uma matriz $\left[\alpha_{i j}\right]$, de ordem $n$, que é mudança entre bases de fibras de $\omega$, a matriz mudança entre as respectivas bases das fibras reais terá ordem $2 n$ e blocos

$$
\left[\begin{array}{cc}
a_{i j} & -b_{i j} \\
b_{i j} & a_{i j}
\end{array}\right]
$$

no lugar de cada número complexo $\alpha_{i j}=a_{i j}+i b_{i j}$.

Agora basta observar que existe uma correspondência entre o conexo $G L_{n}(\mathbb{C})$ e este subconjunto de $G L_{2 n}(\mathbb{R})$, que é um homeomorfismo.

Aplicando este resultado para o caso especial de um fibrado tangente, segue que toda variedade complexa tem uma orientação.

Definição 5.5. Uma métrica hermitiana em um fibrado complexo $\omega$ é uma métrica euclideana em $\omega_{R}$

$$
v \mapsto|v|^{2}
$$

tal que $|i v|=|v|$.

Se $B$ é paracompacto, todo fibrado complexo sobre $B$ admite uma métrica hermitiana.

\subsection{Construção das classes de Chern}

Seja $\omega$ um $n$-fibrado complexo (aqui o consideramos com métrica hermitiana, por simplicidade). Construiremos um $(n-1)$-fibrado $\omega_{0}$ sobre $E_{0}(\omega)$ (os pontos de $E_{0}(\omega)$ serão os pares $(F, v)$, onde $F$ é uma fibra de $\omega$ e $v$ é um vetor não nulo de 
$F)$. A fibra de $\omega_{0}$ sobre o ponto $(F, v)$ é o complemento ortogonal de $v$ em $F$, que tem dimensão $n-1$.

Relembrando a Seqüência de Gysin (Seção 2 do Capítulo 4) para um 2nfibrado vetorial real orientado

$$
\cdots \longrightarrow H^{i-2 n}(B) \stackrel{\cup e\left(\omega_{R}\right)}{\longrightarrow} H^{i}(B) \stackrel{\pi_{0}^{*}}{\longrightarrow} H^{i}\left(E_{0}\right) \longrightarrow H^{i-2 n+1}(B) \longrightarrow \cdots
$$

Se $i<2 n-1, H^{i-2 n}(B)=H^{i-2 n+1}(B)=0, \operatorname{logo} \pi_{0}^{*}: H^{i}(B) \rightarrow H^{i}\left(E_{0}\right)$ é um isomorfismo.

Definimos as classes de Chern $c_{j}(\omega) \in H^{2 j}(B, \mathbf{Z})$ da seguinte forma, indutivamente:

$$
c_{n}(\omega) \doteq e\left(\omega_{R}\right) \in H^{2 n}(B) .
$$

Para $j<n$, temos do comentário acima que $\pi_{0}^{*}: H^{2 j}(B) \rightarrow H^{2 j}\left(E_{0}\right)$ é um isomorfismo, e fazemos então $c_{j}(\omega) \doteq\left(\pi_{0}^{*}\right)^{-1}\left(c_{j}\left(\omega_{0}\right)\right)$, já que $\omega_{0}$ tem dimensão $n-1$.

Para $j>n$, colocamos $c_{j}(\omega)=0$.

Definição 5.6. A classe total de Chern é a soma formal $c(\omega)=1+c_{1}(\omega)+\ldots+c_{n}(\omega)$ pertencente ao anel $H^{*}(B, \mathbf{Z})$.

Seguem agora resultados similares aos axiomas e às propriedades das classes de Stiefel-Whitney. Os dois primeiros podem ser demonstrados por argumentos de indução.

Proposição 5.7 (Naturalidade). Se $f: B \rightarrow \bar{B}$ pode ser levantada a uma aplicação entre fibrados $\omega$ (sobre $B$ ) e $\bar{\omega}$ (sobre $\bar{B}$ ), então

$$
f^{*}(c(\bar{\omega}))=c(\omega)
$$

Proposição 5.8. Se $\varepsilon^{k}$ é o k-fibrado complexo trivial sobre $B$, e $\omega$ também é um fibrado complexo sobre $B$, então $c\left(\varepsilon^{k} \oplus \omega\right)=c(\omega)$.

\subsection{Variedades de Grassmann complexas}

Assim como no caso real, denotaremos por $G_{n}\left(\mathbb{C}^{n+k}\right)$ o conjunto de todos os $n$ planos complexos passando pela origem em $\mathbb{C}^{n+k}$, que possui estrutura de variedade complexa de dimensão $n k$. 
Também denotamos por $\gamma^{n}\left(\mathbb{C}^{n+k}\right)$ o $n$-fibrado complexo sobre a variedade de Grasmann $G_{n}\left(\mathbb{C}^{n+k}\right)$, consistindo dos pares $(X, v)$, com $X \in G_{n}\left(\mathbb{C}^{n+k}\right)$ e $v \in X$.

Para $n=1$, a variedade $G_{1}\left(\mathbb{C}^{k+1}\right)$ é conhecida como o $k$-ésimo espaço projetivo complexo e denotada por $P^{k}(\mathbb{C})$.

Estudaremos agora o anel $H^{*}\left(P^{k}(\mathbb{C}), \mathbf{Z}\right)$. Consideremos a seqüência de Gysin de $\gamma^{1}\left(\mathbb{C}^{k+1}\right)$ sobre $P^{k}(\mathbb{C})$ :

$$
\cdots \longrightarrow H^{i+1}\left(E_{0}\right) \longrightarrow H^{i}\left(P^{k}(\mathbb{C})\right) \stackrel{\cup c_{1}}{\longrightarrow} H^{i+2}\left(P^{k}(\mathbb{C})\right) \stackrel{\pi_{0}^{*}}{\longrightarrow} H^{i+2}\left(E_{0}\right) \longrightarrow \cdots
$$

Observe que $E_{0}(\gamma)=\left\{(L, v): L \in P^{k}(\mathbb{C}), v \in L, v \neq 0\right\}$ tem o mesmo tipo de homotopia de $S^{2 k+1}$, $\log$ para $0 \leq i \leq 2 k-2$, temos a seqüência exata curta:

$$
0 \longrightarrow H^{i}\left(P^{k}(\mathbb{C})\right) \stackrel{\cup c_{1}}{\longrightarrow} H^{i+2}\left(P^{k}(\mathbb{C})\right) \longrightarrow 0
$$

Assim os grupos de cohomologia de ordem par são isomorfos. Além disso, $H^{0}\left(P^{k}(\mathbb{C})\right)=\mathbf{Z}$ e $H^{2 j}\left(P^{k}(\mathbb{C})\right)=\left[c_{1}\left(\gamma^{1}\left(\mathbb{C}^{k+1}\right)\right)^{j}\right], 1 \leq j \leq k$.

Também obtemos que os grupos de cohomologia de ordem ímpar são nulos. Ou seja, $H^{*}\left(P^{k}(\mathbb{C})\right)$ consiste dos polinômios de grau no máximo $k$, gerados por $c_{1}\left(\gamma^{1}\left(\mathbb{C}^{k+1}\right)\right)$.

Podemos fazer agora, como no caso real, $k \rightarrow \infty$, obtendo o $n$-fibrado $\gamma^{n}\left(\mathbb{C}^{\infty}\right)$ (abreviadamente, $\gamma^{n}$ ) sobre a Grassmanniana $G_{n}\left(\mathbb{C}^{\infty}\right)$, (abreviadamente, $G_{n}$ ).

No caso de $n=1$, podemos passar o limite direto, concluindo que $H^{*}\left(G_{1}\right)$ é o anel dos polinômios em $c_{1}\left(\gamma^{1}\right)$. Mais geralmente, temos o seguinte resultado:

Teorema 5.9 (Teorema 14.5). O anel $H^{*}\left(G_{n}, \mathbf{Z}\right)$ é o anel dos polinômios com coeficientes em Z, gerado pelas classes de Chern $c_{1}\left(\gamma^{n}\right), \ldots, c_{n}\left(\gamma^{n}\right)$. Mais ainda, não existem relaçôes polinomiais entre estas classes.

Também enunciamos o resultado que justifica a denominação de fibrado universal para $\gamma^{n}$ e de espaço classificante para $G_{n}$ :

Teorema 5.10 (Teorema 14.6). Para todo n-fibrado complexo $\omega$ com base $B$ paracompacta, existe uma aplicação $f: B \rightarrow G_{n}$, que pode ser levantada a uma aplicação entre os fibrados $\omega$ e $\gamma^{n}$. Mais ainda, quaisquer duas aplicações com esta propriedade são homotópicas.

Um axioma de Stiefel-Whitney que ainda não foi verificado nesta construção das classes de Chern é o Teorema do Produto de Whitney. Para isso, faremos uso do Lema a seguir: 
Lema 5.11. Existe um único polinômio $p_{m, n}$ com $m+n$ variáveis e coeficientes em $\mathbf{Z}$ tal que $c(\omega \oplus \phi)=p_{m, n}\left(c_{1}(\omega), \ldots, c_{m}(\omega), c_{1}(\phi), \ldots, c_{n}(\phi)\right)$, para todo $m$-fibrado complexo $\omega$ e n-fibrado $\phi$ com base paracompacta $B$ comum.

Demonstração. Considere as projeções $\pi_{1}: G_{m} \times G_{n} \rightarrow G_{m}$ e $\pi_{2}: G_{m} \times G_{n} \rightarrow G_{n}$ e os fibrados modelos $\gamma_{1}^{m} \doteq \pi_{1}^{*}\left(\gamma_{m}\right)$ e $\gamma_{2}^{n} \doteq \pi_{2}^{*}\left(\gamma_{n}\right)$ sobre mesma base $G_{m} \times G_{n}$.

A aplicação produto cruzado $H^{*}\left(G_{m}\right) \times H^{*}\left(G_{n}\right) \rightarrow H^{*}\left(G_{m} \times G_{n}\right)$ é um isomorfismo. (De fato, aqui podemos aplicar o isomorfismo de Künneth, pois cada esqueleto de $G_{m}$ e $G_{n}$ é um CW-complexo finito). Assim, $H^{*}\left(G_{m} \times G_{n}\right)$ é o anel de polinômios com coeficientes em $\mathbf{Z}$ gerado por $\pi_{1}^{*}\left(c_{i}\left(\gamma^{m}\right)\right)=c_{i}\left(\gamma_{1}^{m}\right) \doteq c_{i}$ e $\pi_{2}^{*}\left(c_{j}\left(\gamma^{n}\right)\right)=c_{j}\left(\gamma_{2}^{n}\right) \doteq c_{j}^{\prime}$, com $i=1, \ldots, m$ e $j=1, \ldots, n$. Como $c\left(\gamma_{1}^{m} \oplus \gamma_{2}^{n}\right) \in H^{*}\left(G_{m} \times G_{n}\right)$, resulta que

$$
c\left(\gamma_{1}^{m} \oplus \gamma_{2}^{n}\right)=p_{m, n}\left(c_{1}, \ldots, c_{m}, c_{1}^{\prime}, \ldots, c_{n}^{\prime}\right)
$$

para algum polinômio $p_{m, n} \operatorname{com} m+n$ variáveis e coeficientes em $\mathbf{Z}$.

A demonstração do Lema para o caso geral conclui-se aplicando-se o Teorema 5.10.

Agora iremos calcular o polinômio $p_{m, n}$ de (5.2) por indução em $m+n$ (para simplificar a notação, omitiremos o símbolo do produto cup).

Suponha que $c\left(\gamma_{1}^{m-1} \oplus \gamma_{2}^{n}\right)=\left(1+c_{1}+\ldots+c_{m-1}\right)\left(1+c_{1}^{\prime}+\ldots+c_{n}^{\prime}\right)$.

Tome os fibrados $\gamma_{1}^{m-1} \oplus \varepsilon^{1}$ e $\gamma_{2}^{n}$ sobre $G_{m-1} \times G_{n}$. Pelo Lema acima, e pelas Proposição 5.8, temos que $c\left(\gamma_{1}^{m-1} \oplus \varepsilon^{1} \oplus \gamma_{2}^{n}\right)=p_{m, n}\left(c_{1}, \ldots, 0, c_{1}^{\prime}, \ldots, c_{n}^{\prime}\right)$.

Como $c\left(\gamma_{1}^{m-1} \oplus \varepsilon^{1} \oplus \gamma_{2}^{n}\right)=c\left(\gamma_{1}^{m-1} \oplus \gamma_{2}^{n}\right)$, segue que

$$
p_{m, n}\left(c_{1}, \ldots, c_{m}, c_{1}^{\prime}, \ldots, c_{n}^{\prime}\right) \equiv\left(1+c_{1}+\ldots+c_{m}\right)\left(1+c_{1}^{\prime}+\ldots+c_{n}^{\prime}\right) \bmod c_{m}
$$

Analogamente, utilizando a indução com o par $m, n-1$, obtemos:

$$
p_{m, n}\left(c_{1}, \ldots, c_{m}, c_{1}^{\prime}, \ldots, c_{n}^{\prime}\right) \equiv\left(1+c_{1}+\ldots+c_{m}\right)\left(1+c_{1}^{\prime}+\ldots+c_{n}^{\prime}\right) \bmod c_{n}^{\prime}
$$

Agora, note que $\mathbf{Z}\left[c_{1}, \ldots, c_{m}, c_{1}^{\prime}, \ldots, c_{n}^{\prime}\right]$ é um domínio de fatoração única, logo (5.3) e (5.4) dão

$$
u \cdot c_{m} \cdot c_{n}=p_{m, n}\left(c_{1}, \ldots, c_{m}, c_{1}^{\prime}, \ldots, c_{n}^{\prime}\right)-\left(1+c_{1}+\ldots+c_{m}\right)\left(1+c_{1}^{\prime}+\ldots+c_{n}^{\prime}\right) .
$$


Analisando, vemos que a dimensão de $u$ deve ser zero, ou seja, um elemento de Z. Mais ainda, olhando para a última classe, e usando as propriedades da classe de Euler (Proposição 4.5), temos que

$$
c_{m} \cdot c_{n}^{\prime}+u \cdot c_{m} \cdot c_{n}^{\prime}=c_{m+n}\left(\gamma_{1}^{m} \oplus \gamma_{2}^{n}\right)=e\left(\gamma_{1 R}^{m}\right) \cdot e\left(\gamma_{2 R}^{n}\right)=c_{m} \cdot c_{n}^{\prime}
$$

Assim, $u=0$, como queríamos demonstrar.

\section{$5.4 \quad$ Fibrados duais (conjugados)}

Seja $\omega$ um $n$-fibrado vetorial complexo. Definimos um novo fibrado $\bar{\omega}$ (conjugado) tal que $\bar{\omega}_{R}=\omega_{R}$ (mesmo espaço total), mas com estrutura complexa oposta nas fibras. Desta forma, a aplicação identidade em $E(\omega)$ é linear conjugada.

Lema 5.12. $c_{k}(\bar{\omega})=(-1)^{k} c_{k}(\omega)$.

Demonstração. Basta notar que os fibrados têm mesma orientação se $n$ é par e orientação oposta se $n$ é ímpar e daí aplicamos indução. Para classe de ordem $n$, a classe de Euler de $\omega_{R}$, é imediato. Se $k<n$, fazemos o procedimento padrão:

$$
\begin{aligned}
c_{k}(\omega) & =\left(\pi_{0}^{*}\right)^{-1} c_{k}\left(\omega_{0}\right) \\
& =\left(\pi_{0}^{*}\right)^{-1}(-1)^{k} c_{k}\left(\overline{\omega_{0}}\right) \\
& =(-1)^{k}\left(\pi_{0}^{*}\right)^{-1} c_{k}\left((\bar{\omega})_{0}\right)=(-1)^{k} c_{k}(\bar{\omega}) .
\end{aligned}
$$

Dado o mesmo fibrado $\omega$, podemos também definir um fibrado $\operatorname{Hom}(\omega, \mathbb{C})$ (dual) com mesma base e com fibra típica $\operatorname{Hom}(F, \mathbb{C})$, para cada fibra $F$ de $\omega$. Se $\omega$ possui métrica hermitiana, então $\operatorname{Hom}(\omega, \mathbb{C}) \simeq \bar{\omega}$. De fato, a correspondência $v \mapsto<\cdot, v>\operatorname{de} \bar{F}$ em $\operatorname{Hom}(F, \mathbb{C})$ é um isomorfismo entre as respectivas fibras, pelo teorema da representação de Riesz.

Teorema 5.13. A classe total de Chern do fibrado tangente a $P^{n}(\mathbb{C})$ vale $c\left(\tau_{P^{n}}\right)=$ $(1+a)^{n+1}$, onde a é um gerador de $H^{2}\left(P^{n}(\mathbb{C}), \mathbf{Z}\right)$ (na verdade, $a=-c_{1}\left(\gamma^{1}\left(\mathbb{C}^{k+1}\right)\right)$ ).

Demonstração. Seja $\omega^{n}$ o complemento ortogonal de $\gamma^{1}\left(\mathbb{C}^{n+1}\right)$ usando a métrica usual em $\mathbb{C}^{n+1}$, isto é, $\gamma^{1}\left(\mathbb{C}^{n+1}\right) \oplus \omega^{n}=\varepsilon^{n+1}$, o fibrado trivial sobre $P^{n}(\mathbb{C})$.

Agora, se $L$ é uma reta passando pela origem em $\mathbb{C}^{n+1}$ e $L^{\perp}$ é seu complemento, $\operatorname{Hom}\left(L, L^{\perp}\right)$ pode ser identificado com as retas que podem ser consideradas gráficos de aplicações lineares de $L$ em $L^{\perp}$. Isto mostra que o fibrado $\operatorname{Hom}\left(\gamma^{1}\left(\mathbb{C}^{n+1}\right), \omega^{n}\right)$ pode ser identificado com $\tau_{P^{n}}$. Assim, temos: 


$$
\begin{aligned}
& \tau_{P^{n}} \oplus \varepsilon^{1} \simeq \operatorname{Hom}\left(\gamma^{1}\left(\mathbb{C}^{n+1}\right), \omega^{n} \oplus \gamma^{1}\left(\mathbb{C}^{n+1}\right)\right) \simeq \oplus_{n+1} \operatorname{Hom}\left(\gamma^{1}\left(\mathbb{C}^{n+1}\right), \varepsilon^{1}\right) \simeq \\
& \simeq \oplus_{n+1} \overline{\gamma^{1}\left(\mathbb{C}^{n+1}\right)}
\end{aligned}
$$

Logo, $c\left(\tau_{P^{n}}\right)=\left(1-c_{1}\left(\gamma^{1}\left(\mathbb{C}^{n+1}\right)\right)\right)^{n+1}$.

Observação 5.14. Segue do último teorema que $c_{n}\left(\tau_{P^{n}}\right)=(n+1) a^{n}$.

Logo, avaliando na classe fundamental de homologia ,

$$
\chi\left[P^{n}(\mathbb{C})\right]=<c_{n}\left(\tau_{P^{n}}\right), \mu_{2 n}>=(n+1)<a^{n}, \mu_{2 n}>=( \pm 1)(n+1) .
$$

Mas, por definição, $\chi\left[P^{n}(\mathbb{C})\right]=n+1$, e assim temos que $a^{n}$ é o gerador de $H^{2 n}\left(P^{n}(\mathbb{C}), \mathbf{Z}\right)$ compatível com a orientação escolhida. 


\section{Capítulo 6}

\section{Obstruções}

Stiefel e Whitney (independentemente) construíram as classes características de variedades suaves como obstrução à existência de seções linearmente independentes do fibrado tangente. É esta a abordagem que será dada neste capítulo. As referências serão [3] e [18].

Seja $\xi$ um $n$-fibrado vetorial com base $B$, um CW-complexo. Nosso objetivo está nas seguintes questões: dado $1 \leq k \leq n$, é possível construir $k$ seções linearmente independentes do espaço total $E$ ? Fazendo a construção crescente de $B$ por esqueletos, até que dimensão podemos fazer a construção destas seções?

Para cada fibra $F$ de $\xi$, vamos considerar a variedade de Stiefel $V_{k}(F)$ consistindo de todas as $k$-uplas de vetores linearmente independentes de $F$. Denotamos por $V_{k}(\xi)$ o fibrado com base $B$ e fibra típica $V_{k}(F)$. Uma seção deste fibrado representa $k$ seções linearmente independentes de $\xi$.

Considere agora a seguinte situação: $B$ admite uma triangulação $(K)$ de tal forma que todo simplexo $\sigma$ de $(K)$ está num aberto $U$ da trivialização local de $V_{k}(\xi)$. Suponha que exista uma seção $v^{k}$ de $V_{k}(\xi)$ definida em $\partial \sigma$, o bordo de um simplexo de dimensão $j$. Sob quais condições seria possível estender a seção ao interior de $\sigma$ ? (vide Figura 6.1)

Como o aberto $U$ satisfaz a trivialização local, temos que $V_{k}(\xi)_{\mid U} \simeq U \times V_{k}(F)$. Sendo $p_{2}$ a projeção sobre o segundo fator, temos a seguinte composição:

$$
\partial \sigma \stackrel{v^{k}}{\longrightarrow} V_{k}(\xi)_{\mid U} \simeq U \times V_{k}(F) \stackrel{p_{2}}{\longrightarrow} V_{k}(F)
$$

o que, no fundo, nos dá uma aplicação de $S^{j-1} \simeq \partial \sigma \rightarrow V_{k}(F)$. Portanto, temos um elemento $\left[\gamma\left(v^{k}, \sigma\right)\right]$ do grupo de homotopia $\pi_{j-1}\left(V_{k}(F)\right)$. É necessário conhecer estes grupos para verificarmos a obstrução. Eles foram calculados por Stiefel e Whitney (vide [24]). No caso real, temos: 


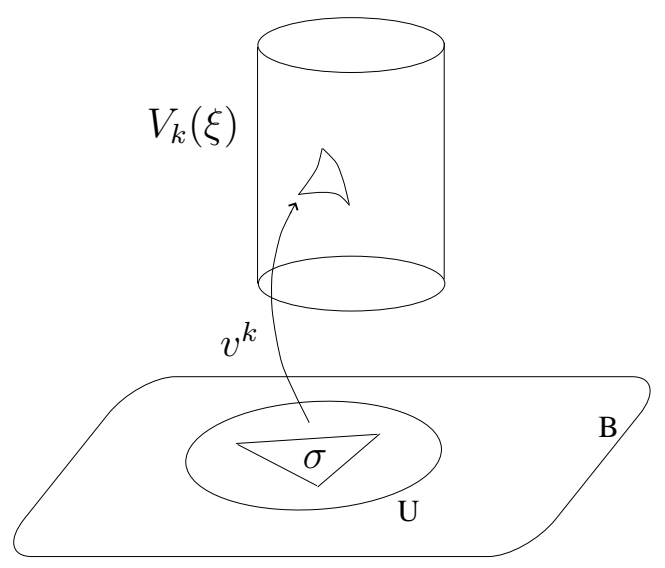

FIGURA 6.1: Estendendo ao interior de $\sigma$

$$
\pi_{j-1}\left(V_{n-j+1}(F)\right)=\left\{\begin{array}{rll}
\mathbf{Z}_{2}, & \text { se } & j \text { par } e j<n \\
\text { cíclico infinito, } & \text { se } & j \text { ímpar ou } j=n \\
0, & \text { nos demais casos. }
\end{array}\right.
$$

Para o caso de um fibrado vetorial complexo, temos que

$$
\pi_{j-1}\left(V_{k}(F)\right)=\left\{\begin{array}{ccc}
0, & \text { se } & j<2 n-2 k+1 \\
\mathbf{Z}, & \text { se } & j=2(n-k+1) .
\end{array}\right.
$$

Denote por $2 p=2(n-k+1)$. Um gerador de $\pi_{2 p-1}\left(V_{k}(F)\right)$ é construído da seguinte forma: escolha um $(n-p)$-referencial em $\mathbb{C}^{n}$. A esfera unitária $S^{2 p-1}$ de $\mathbb{C}^{p}$ é orientada naturalmente. Considere para todo ponto desta esfera o $(n-p+1)$ referencial formado pelo vetor $w$ e pelo $(n-p)$-referencial fixado. Esta aplicação de $S^{2 p-1}$ em $V_{k}(F)$ define um gerador de $\pi_{2 p-1}\left(V_{k}(F)\right)$.

\subsection{Definição das classes de Chern por obstrução}

Considere o caso de uma variedade analítica complexa $B$ e seu fibrado tangente. Para todo simplexo $\sigma$, de dimensão $j$, uma seção $v$ sobre o bordo de $\sigma$ define um elemento $[\gamma(v, \sigma)]$. Mas, pelos cálculos dos grupos acima, não existe obstrução à construção de $k$ referenciais sobre os esqueletos de dimensão menor do que $2(n-k+1)$ (dimensão de obstrução). Quando chegamos a esta dimensão, obtemos um elemento $[\gamma(v, \sigma)] \in \pi_{2 p-1}\left(V_{k}(F)\right) \simeq \mathbf{Z}$.

Pode-se estender o campo $v$, definido em $\partial \sigma$, através de uma homotetia centrada no baricentro de $\sigma$ (denotado por $\hat{\sigma}$ ) com singularidade isolada no 
mesmo. Calculamos o índice do campo $v$ estendido, $I(v, \hat{\sigma})$, cujo valor é $I(v, \hat{\sigma})=[\gamma(v, \sigma)] \in \mathbf{Z}$.

Definimos assim a cocadeia

$$
[\gamma(v)]=\sum_{\operatorname{dim}(\sigma)=2 p} I(v, \hat{\sigma}) \sigma^{*}
$$

onde $\sigma^{*}$ é a cocadeia cujo valor é 1 sobre a cadeia $\sigma$ e 0 para as demais. Esta cocadeia é um cociclo, denominado cociclo de obstrução, e sua classe é denominada classe de obstrução ou p-ésima classe de Chern de B. Esta classe independe da cobertura trivializadora da variedade, da triangulação e da escolha do campo $v$ inicial ([24]).

\subsection{Relação com as classes de Stiefel-Whitney}

Considere agora o caso real e coloque $j=n-k+1$. Steenrod em [24] mostra que é possível construir uma seção de $V_{k}(\xi)$ sobre o $(n-k)$-esqueleto de $B$. Mas só é possível construir uma seção sobre o $(n-k+1)$-esqueleto se, e somente se, a classe de obstrução $v_{j}(\xi) \in H^{j}\left(B, \pi_{j-1}\left(V_{k}(F)\right)\right)$ é nula.

Calculados os grupos $\pi_{j-1}\left(V_{n-j+1}(F)\right)$, podemos considerar o homomorfismo induzido $h_{*}: H^{j}\left(B, \pi_{j-1}\left(V_{n-j+1}(F)\right)\right) \rightarrow H^{j}\left(B, \mathbf{Z}_{2}\right)$ que reduz os coeficientes módulo 2. Temos que:

Teorema 6.1. $h_{*}\left(v_{j}(\xi)\right)=w_{j}(\xi)$.

Demonstração. Tomando o fibrado universal $\gamma^{n}$ sobre a Grasmmanniana $G_{n}$, temos que $h_{*}\left(v_{j}\left(\gamma^{n}\right)\right)$, como elemento de $H^{j}\left(G_{n}, \mathbf{Z}_{2}\right)$, pode ser escrito como $f\left(w_{1}\left(\gamma^{n}\right), \ldots, w_{n}\left(\gamma^{n}\right)\right)$, para algum polinômio de grau $n$ com coeficientes em $\mathbf{Z}_{2}$.

É mostrado em [24], tópico (35.7), que, assim como as classes de StiefelWhitney, a classe de obstrução também é natural em relação a aplicações entre fibrados. Como existe uma tal aplicação entre $\gamma^{n}$ e $\xi$, isto nos diz que $h_{*}\left(v_{j}(\xi)\right)=f\left(w_{1}(\xi), \ldots, w_{n}(\xi)\right)$. Separando a classe de maior grau, escrevemos $h_{*}\left(v_{j}(\xi)\right)=f^{\prime}\left(w_{1}(\xi), \ldots, w_{j-1}(\xi)\right)+\lambda \cdot w_{j}(\xi)$, onde $\lambda \in \mathbf{Z}_{2}$.

Considere agora o $n$-fibrado $\eta=\gamma^{j-1} \oplus \varepsilon^{n-j+1}$ sobre $G_{j-1}$. Sabemos que $\eta$ admite $n-j+1$ seções linearmente independentes, $\operatorname{logo} v_{j}(\eta)=0$. Assim, como a identidade acima vale para todo $n$-fibrado, temos que $0=f^{\prime}\left(w_{1}(\eta), \ldots, w_{j-1}(\eta)\right)+$ $\lambda \cdot w_{j}(\eta)$. Como $w(\eta)=w\left(\gamma^{j-1}\right)$, segue que

$$
f^{\prime}\left(w_{1}\left(\gamma^{j-1}\right), \ldots, w_{j-1}\left(\gamma^{j-1}\right)\right)=0
$$


Mas estas classes são algebricamente independentes, $\operatorname{logo} f^{\prime} \equiv 0$. Resta então mostrar que $\lambda=1$.

Detalharemos este procedimento apenas para o caso $j=n$. Restrinja o fibrado $\gamma^{n}$ sobre $P^{n}$. A fibra $F$ em cada classe de pontos $\{u,-u\}$, com $u \in S^{n}$ é o subespaço $\left\{v \in \mathbb{R}^{n+1}: u \cdot v=0\right\}$. Fixe $u_{0} \in S^{n}$. Definimos assim uma seção do fibrado $\gamma^{n}$ : a cada $\{u,-u\} \in P^{n}$, associamos o vetor $u_{0}-\left(u_{0} \cdot u\right) u \in F$. Esta seção é nula apenas em $\left\{u_{0},-u_{0}\right\}$. Desta forma temos uma seção $v$ de $V_{1}\left(\gamma^{n}\right)$ sobre o $(n-1)$-esqueleto de $P^{n}$, que denotaremos por $\partial \sigma$ (vide figura).

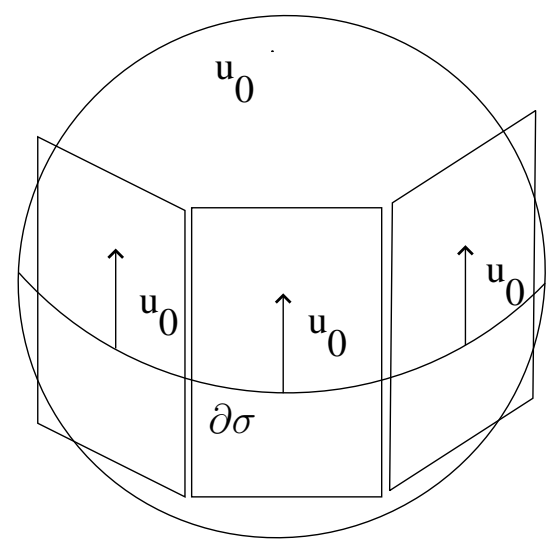

FIGURA 6.2: Seção constante sobre $\partial \sigma$

Note que $v$ associa $u_{0}$ a cada ponto de $\partial \sigma$. Da construção da obstrução da seção anterior, segue que $v_{n}\left(\gamma^{n}\right)(\sigma)=[\gamma(v, \sigma)]$, onde $\gamma(v, \sigma)=p_{2} \circ v: S^{n-1} \simeq \partial \sigma \rightarrow$ $V_{1}(F)$ é um gerador de $\pi_{n-1}\left(V_{1}(F)\right)=\pi_{n-1}(F-0) \simeq \mathbf{Z}$. Logo $v_{n}\left(\gamma^{n}\right)(\sigma)= \pm 1$. Assim, $h_{*}\left(v_{n}\left(\gamma^{n}\right)\right) \neq 0$ e daí, o valor de $\lambda$ só pode ser 1 .

\subsection{Relação com a classe de Euler}

Vimos que a última classe de obstrução $v_{n}(\xi)$ é um elemento do grupo $H^{n}\left(B, \pi_{n-1}\left(V_{1}(F)\right)\right.$. Como $\pi_{n-1}\left(V_{1}(F)\right)=\pi_{n-1}(F-0) \simeq \mathbf{Z}$, faz sentido o seguinte resultado:

Teorema 6.2. Se $\xi$ é um n-fibrado orientável sobre um $C W$-complexo $B$, então $v_{n}(\xi)=e(\xi)$. 
Demonstração. Considere inicialmente a aplicação $\pi_{0}: E_{0} \rightarrow B$ e o fibrado induzido $\pi_{0}^{*}(\xi)$ sobre $E_{0}$. Lembremos de (1.1) que o espaço total $E_{1}$ deste fibrado consiste dos elementos $\left(e^{\prime}, e\right) \in E_{0} \times E$ tais que $\pi_{0}\left(e^{\prime}\right)=\pi(e)$. Definimos assim uma seção não-nula, do fibrado induzido, $s: E_{0} \rightarrow E_{1}$, onde $s\left(e^{\prime}\right)=\left(e^{\prime}, e^{\prime}\right)$. Logo $v_{n}\left(\pi_{0}^{*}(\xi)\right)=0$. Como a obstrução obedece a naturalidade, temos que $\pi_{0}^{*}\left(v_{n}(\xi)\right)=v_{n}\left(\pi_{0}^{*}(\xi)\right)=0$. Aplicando a seqüência de Gysin, segue que $v_{n}(\xi) \in \operatorname{Ker}\left(\pi_{0}^{*}\right)=\operatorname{Im}(\cup e(\xi))$. Assim, $v_{n}(\xi)=\lambda \cup e(\xi)=\lambda \cdot e(\xi)$, onde $\lambda \in \mathbf{Z}$. Tomando o fibrado universal orientável sobre $\tilde{G}_{n}$, pela naturalidade das classes, vemos que $\lambda$ independe do fibrado orientável $\xi$.

Agora, reduzimos módulo 2 os lados da igualdade $v_{n}\left(\tilde{\gamma}^{n}\right)=\lambda e\left(\tilde{\gamma}^{n}\right)$, obtendo, pelo teorema anterior, que $w_{n}\left(\tilde{\gamma}^{n}\right)=\lambda w_{n}\left(\tilde{\gamma}^{n}\right)$.

Sabemos que $w_{n}\left(\tilde{\gamma}^{n}\right) \neq 0$, se $n>1$ (Se $n=1$, então $H^{1}\left(\tilde{G}_{1}, \mathbf{Z}_{2}\right)=0$, logo se considerarmos a aplicação de fibrados $f$ entre $\xi$ e $\tilde{\gamma}^{n}$, temos que $e(\xi)=f^{*}(0)=0$ e o resultado seguiria imediatamente).

Então, concluímos que $\lambda$ é ímpar. Daí, para algum $k \in \mathbf{Z}, v_{n}(\xi)=(2 k+$

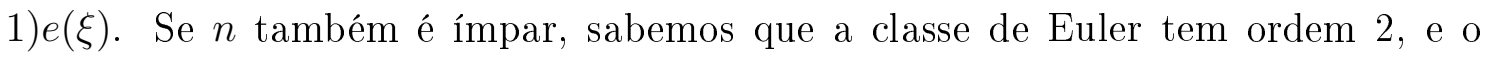
resultado também estaria provado.

Considere então que $n$ é par. Basta provarmos que $\lambda=1$ para algum fibrado orientável de ordem $n$. Seja $\tau$ o fibrado tangente a $S^{n}$. Sabemos do Corolário 4.17 que a avaliação da classe de Euler sobre a classe fundamental da variedade dá a característica de Euler: $\left\langle e(\tau), \mu>=\chi\left(S^{n}\right)=2\right.$.

Steenrod, em [24], tópico (39.7), prova uma fórmula análoga para a classe de obstrução:

$$
<v_{n}(\tau), \mu>=\chi\left(S^{n}\right)=2 .
$$

Daí, temos que $\lambda<e(\tau), \mu>=\chi\left(S^{n}\right)=2$, o que implica que $\lambda=1$.

Observação 6.3. Note que este resultado nos dá uma recíproca da Proposição 4.6. 


\section{Capítulo 7}

\section{Estratificações de Whitney}

A partir de agora, iniciamos o estudo das variedades singulares. Nos capítulos seguintes, ao definir as classes de Chern para este tipo de variedade, necessitaremos particioná-la em subconjuntos regulares e considerar o espaço tangente a cada estrato. O objetivo deste capítulo é estudar as propriedades da estratificação Whitney regular. A referência utilizada aqui é [7].

\subsection{Conjuntos Algébricos}

Seja $M$ uma variedade suave e $V$ um subconjunto de $M$. Uma estratificação de $V$ é uma partição $\wp$ de $V$ em subvariedades suaves de $M$ (estratos), satisfazendo:

(i) (Condição de finitude local) para todo ponto $p \in V$, existe uma vizinhança de $p$ em $M$ que intercepta apenas um número finito de estratos.

(ii) (Condição de fronteira - exigida em algumas ocasiões) Se $X$ e $Y$ são estratos, com $X \cap \bar{Y} \neq \emptyset$, então $X \subset \bar{Y}$ (ou seja, a fronteira de um estrato é reunião de estratos)

Definição 7.1. Um conjunto algébrico (ou uma variedade algébrica) é o conjunto de zeros de um número finito de polinômios em $\mathbb{R}^{m}$. Tal conjunto será dito irredutível se não puder ser escrito como reunião de subconjuntos próprios algébricos.

Exemplo 7.2. O guarda-chuva de Whitney (figura a seguir) é o conjunto de zeros do polinômio $f(x, y, z)=x^{2}-z y^{2}$. Podemos calcular seus pontos singulares (igualando o vetor gradiente a zero) e encontrar como resposta o eixo $z$. Uma estratificação do guarda-chuva consiste dos semi-eixos $z>0$ e $z<0$ (para assegurar a condição (ii)), da origem e da parte regular. 


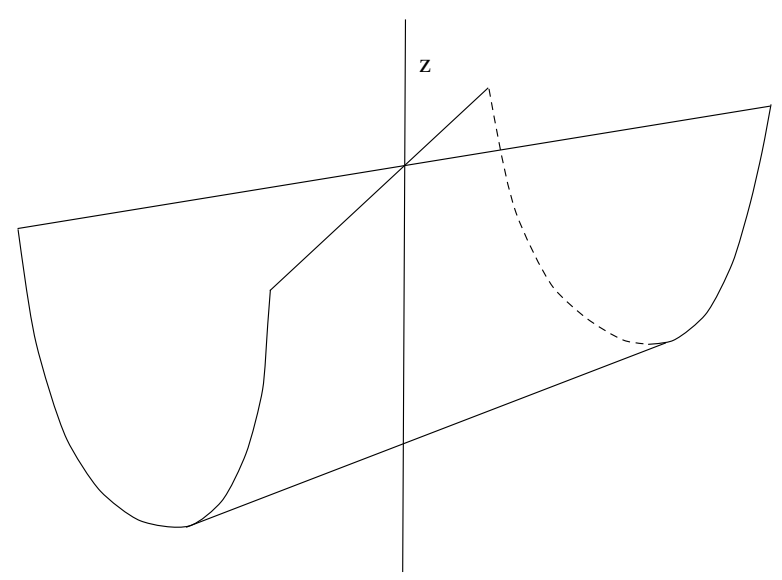

FIGURA 7.1: Guarda-chuva de Whitney

Sabe-se que o conjunto de pontos singulares $\sum V$ de $V$ algébrico também é algébrico, de dimensão estritamente menor. Também, $V-\sum V$ é uma variedade suave.

Considere a seguinte filtração de $V: V_{d} \supseteq V_{d-1} \supseteq \ldots$, onde $V_{d}=V$ e $d=\operatorname{dim}(V)$. Coloque $V_{i-1}=\sum V_{i}$, se $\operatorname{dim}\left(V_{i}\right)=i$, ou $V_{i-1}=V_{i}$, caso contrário.

Existem então um número finito de diferenças $V_{i}-V_{i-1}$, cada uma sendo variedade suave ou vazia. Assim, todo conjunto algébrico admite uma estratificação.

Whitney buscava estratificações para as quais o tipo topológico de cada estrato fosse o mesmo. Isso ocorre no exemplo acima, mas não ocorre, por exemplo, quando a origem não é um estrato.

Para evitar tais situações, Whitney introduziu as seguintes condições de regularidade.

Sejam $X$ e $Y$ estratos de $\wp$, estratificação de $V$ (pensando localmente, imaginamos aqui $\left.V \subseteq \mathbb{R}^{m}\right)$. Sejam $\left(x_{n}\right)$ uma seqüência em $X$ e $\left(y_{n}\right)$ uma seqüência em $Y \operatorname{com} x_{n} \neq y_{n}$ e $x_{n}, y_{n} \rightarrow x \in X$. Dizemos que $Y$ é Whitney-regular sobre $X$ em $x \in X$ se:

(a) (Tangentes) Se $T_{y_{n}} Y$ converge em Grasmmanniana para um plano $T$, então $T_{x} X \subseteq T$;

(b) (Secantes) Se as retas $\overline{x_{n} y_{n}}$ convergem a uma reta $l$ e $T_{y_{n}} Y \rightarrow T$, então $l \subset T$.

Pode-se mostrar, que, na realidade, a condição (b) implica a condição (a).

Diz-se que $Y$ é Whitney-regular sobre $X$ se o for em cada ponto de $X$, e que a estratificação é de Whitney se cada estrato o for sobre os demais. 
No exemplo acima, se não subdividíssemos o eixo z, não seria verificada a condição (b) na origem.

Lema 7.3 (Teorema 1.1). Sejam $X, Y$ variedades suaves de $M$ e $x \in X \cap \overline{(Y-X)}$. Suponha também que $Y$ é Whitney-regular sobre $X$ em $x$. Então $\operatorname{dim}(X)<\operatorname{dim}(Y)$.

Se $\wp_{1}, \ldots, \wp_{n}$ são estratificações de Whitney de $V_{1}, \ldots, V_{n}$, podemos obter uma estratificação de Whitney (Produto) de $V_{1} \times \ldots \times V_{n}$ tomando como estratos as variedades suaves $X_{1} \times \ldots \times X_{n}$ onde $X_{i} \in \wp_{i}, i=1, \ldots, n$.

Os próximos lemas também mostram como construir novas estratificações de Whitney a partir de algumas já dadas.

As variedades suaves $X_{1}, \ldots, X_{n}$ estão em posição geral quando a aplicação $T_{x} M \rightarrow \oplus \frac{T_{x} M}{T_{x} X_{i}}$ é sobrejetora, para qualquer $x \in X_{1} \cap \ldots \cap X_{n}$. Neste caso, $X_{1} \cap \ldots \cap X_{n}$ é uma variedade suave.

Sejam agora $\wp_{1}, \ldots, \wp_{n}$ estratificações de $V_{1}, \ldots, V_{n}$. Dizemos que $\wp_{1}, \ldots, \wp_{n}$ estão em posição geral quando $X_{1}, \ldots, X_{n}$ estiverem, onde $X_{i} \in \wp_{i}, i=1, \ldots, n$. Neste caso, $X_{1} \cap \ldots \cap X_{n}$ será um estrato da estratificação $\wp_{1} \cap \ldots \cap \wp_{n}$ de $V_{1} \cap \ldots \cap V_{n}$.

Lema 7.4. Nessas condições, se $\wp_{i}$ for estratificação de Whitney de $V_{i}$, então $\wp_{1} \cap$ $\ldots \cap \wp_{n}$ será estratificação de Whitney de $V_{1} \cap \ldots \cap V_{n}$.

Demonstração. Coloquemos $X=X_{1} \cap \ldots \cap X_{n}$ e $Y=Y_{1} \cap \ldots \cap Y_{n}$ dois estratos da estratificação intersecção. Sejam $x \in X$ e $x_{i}, y_{i} \rightarrow x$, com $x_{i} \in X$ e $y_{i} \in Y$ tais que $\overline{x_{i} y_{i}} \rightarrow l$ e também $T_{y_{i}} Y \rightarrow T \subseteq \mathbb{R}^{m}$ (hipóteses das condições de Whitney).

Devemos mostrar que $l \subset T$. Usando a compacidade das Grasmmannianas, assumimos que $T_{y_{i}} Y_{s} \rightarrow T_{s}, s=1, \ldots, n$. Como $Y_{s}$ é Whitney-regular sobre $X_{s}$ em $x$, para todo $s$, então, $l \subseteq \cap T_{s}$. Vamos mostrar que $\cap T_{s}=T$

Inicialmente, temos, pela hipótese de posição geral, que $\cap T_{y_{i}} Y_{s}=T_{y_{i}} Y$, o que, no limite, dá

$$
\cap T_{s} \supseteq T
$$

Pela regularidade, $T_{s} \supseteq T_{x} X_{s}$, que estão em posição geral, $\operatorname{logo} T_{1}, \ldots, T_{n}$ também estão. Portanto,

$$
\operatorname{dim}\left(\cap T_{s}\right)=\operatorname{dim}\left(\cap T_{y_{i}} Y_{s}\right)=\operatorname{dim}\left(T_{y_{i}} Y\right)=\operatorname{dim}(T)
$$

Com esta igualdade e (7.1), terminamos a prova.

Seja $\wp^{\prime}$ uma estratificação de $V^{\prime} \subseteq M^{\prime}$ e $f: M \rightarrow M^{\prime}$ transversal a $\wp^{\prime}$ (transversal a cada estrato $X^{\prime}$ de $\wp^{\prime}$, isto é, $d_{x} f\left(T_{x} M\right)+T_{f(x)} X^{\prime}=T_{f(x)} M$, para 
todo $x \in M$ tal que $\left.f(x) \in X^{\prime}\right)$. Obtemos uma estratificação $\wp$ induzida, de $f^{-1}\left(V^{\prime}\right)$, tomando como estratos as variedades suaves $f^{-1}\left(X^{\prime}\right)$.

Lema 7.5. Nessas condições, se $\wp^{\prime}$ for estratificação de Whitney de $V^{\prime}$, então $\wp$ será estratificação de Whitney de $V=f^{-1}\left(V^{\prime}\right)$.

Demonstração. Escreva $f$ como composição de $F: M \rightarrow g r a f(f), F(x)=(x, f(x))$ com a projeção $\pi: M \times M^{\prime} \rightarrow M^{\prime}$. Temos que $F$ leva $\wp$ difeomorficamente numa estratificação $\wp "$ de um subconjunto de graf $(f)$. Logo basta mostrar que $\wp "$ é Whitney.

Por hipótese, a estratificação em $M \times V^{\prime}$ é transversal ao $\operatorname{graf}(f)$. Aplicando o Lema anterior, segue que $\left(M \times V^{\prime}\right) \cap \operatorname{graf}(f)$ é Whitney. Mas esta estratificação é justamente $\wp^{\prime \prime}$.

Um caso particular deste Lema está em se considerar $\wp$ uma estratificação de $V \subseteq M$ e $U$ um aberto de $M$. Como a inclusão $U \rightarrow M$ é transversal a $\wp$, segue que existe uma estratificação induzida em $U \cap V$ denotada por $\wp_{U}$ (restrição a $U$ ).

Outros tópicos estudados em estratificações são buscar unicidades de estratificações e refinamentos de estratificações. Neste último caso, uma saída é definir filtrações por dimensão, e introduzir uma relação de ordem parcial nas estratificações de um dado conjunto, de forma a tentar encontrar um elemento minimal.

\subsection{Conjuntos Semi-algébricos}

Procuramos aqui uma classe útil, e relativamente grande, de conjuntos admitindo estratificação de Whitney.

Definição 7.6. A classe dos conjuntos semi-algébricos de $\mathbb{R}^{m}$ é definida como a menor álgebra de Boole que contém os conjuntos da forma $\left\{x \in \mathbb{R}^{m}: f(x)>0\right\}$, $f: \mathbb{R}^{m} \rightarrow \mathbb{R}$ polinomial.

Assim, essa classe é preservada por reuniões finitas, complementares e produtos cartesianos.

Lema 7.7. Seja $p: \mathbb{R}^{n} \rightarrow \mathbb{R}^{k}$ uma aplicação polinomial. Se A é semi-algébrico em $\mathbb{R}^{k}$, então $p^{-1}(A)$ é semi-algébrico em $\mathbb{R}^{n}$.

Demonstração. Notemos que $\left\{p^{-1}(A)\right\}$, com $A$ semi-algébrico em $\mathbb{R}^{k}$ é uma álgebra de Boole em $\mathbb{R}^{n}$ que contém os conjuntos da forma $p^{-1}\left(\left\{x \in \mathbb{R}^{k}: f(x)>0\right\}\right)$, 
com $f: \mathbb{R}^{k} \rightarrow \mathbb{R}$ polinomial. Logo, contém $\left\{y \in \mathbb{R}^{n}:(f \circ p)(y)>0\right\}$ (veja que $f \circ p: \mathbb{R}^{n} \rightarrow \mathbb{R}$ é polinomial) e é a menor álgebra de Boole com esta propriedade. Assim, $\left\{p^{-1}(A)\right\}$, com $A$ semi-algébrico em $\mathbb{R}^{k}$ está contido no conjunto dos semialgébricos em $\mathbb{R}^{n}$.

Um resultado parecido, mas não tão simples de se mostrar é o seguinte:

Teorema 7.8 (Tarski-Seidenberg, Teorema 2.1). A imagem de um conjunto semialgébrico em $\mathbb{R}^{n}$ pela aplicação polinomial $p: \mathbb{R}^{n} \rightarrow \mathbb{R}^{k}$ é um conjunto semi-algébrico $e m \mathbb{R}^{k}$.

Da demonstração deste resultado, dada em [14], segue que um conjunto semialgébrico tem um número finito de componentes conexas, cada qual também semialgébrica.

Lema 7.9. O fecho de um conjunto $A$, semi-algébrico em $\mathbb{R}^{m}$, também é semialgébrico.

Demonstração. Escreva $B=\left\{(x, y, \varepsilon) \in A \times \mathbb{R}^{m} \times \mathbb{R}:\|x-y\|<\varepsilon\right\}$ e $C=\{(x, y, \varepsilon) \in$ $\left.\mathbb{R}^{m} \times \mathbb{R}^{m} \times \mathbb{R}:\|x-y\|^{2}<\varepsilon^{2}\right\}$. Sendo o polinômio $f: \mathbb{R}^{m} \times \mathbb{R}^{m} \times \mathbb{R} \rightarrow \mathbb{R}$ tal que $f(x, y, \varepsilon)=\varepsilon^{2}-\|x-y\|^{2}$, temos que $C=f^{-1}(0, \infty)$, logo $C$ é semi-algébrico. Como $B=C \cap\left[A \times \mathbb{R}^{m} \times \mathbb{R}\right], B$ também é semi-algébrico.

Agora resta apenas manipular $\bar{A}$ como combinação de produtos, diferenças e projeções de $B$.

Enunciamos um resultado útil, encontrado em [6], Lema 1.10:

Proposição 7.10. Qualquer estrato semi-algébrico $Y$ é Whitney-regular sobre um estrato de dimensão nula.

Seja $V \subset M$, com $M$ variedade suave. Um ponto $x \in V$ é regular, de dimensão $d$, se possui uma vizinhança $U$ em $M$ tal que $U \cap V$ é variedade suave de dimensão $d$. Se $V$ tem ao menos um ponto regular, a dimensão de $V$ é definida como a máxima dentre as de seus pontos regulares.

Um resultado importante é o de que todo conjunto semi-algébrico nãovazio possui ao menos um ponto regular. Na verdade, esses pontos formam um subconjunto denso ([7], Teorema 2.4). Logo a dimensão está bem definida para conjuntos semi-algébricos.

Os pontos de um conjunto semi-algébrico $V$ que não são regulares de dimensão máxima são chamados singulares, e denotados por $\sum V$. O conjunto singular também é semi-algébrico, de dimensão estritamente menor que a de $V$ ([7], Teorema 2.5). 
Definição 7.11. Sejam $X, Y$ subvariedades suaves de $M$. Definimos o conjunto mau $B(X, Y)=\{x \in X: Y$ não é Whitney regular sobre $X\}$.

Se $X$ e $Y$ forem variedades semi-algébricas de $\mathbb{R}^{m}$, então $B(X, Y)$ também é semi-algébrico, de dimensão estritamente menor que a de $X$ ([7], Teorema 2.6).

Definição 7.12. Sejam $P, Q$ conjuntos semi-algébricos em $\mathbb{R}^{m}$. Definimos o conjunto $W(P, Q)=\sum P \cup B\left(P-\sum P, Q-\sum Q\right)$.

Note que a definição acima é compatível com a anterior. Além disso, este conjunto $W(P, Q)$ também é semi-algébrico, de dimensão estritamente menor que a de $P$. Definidas todas estas ferramentas, podemos mostrar o resultado a seguir, objetivo principal deste capítulo.

Teorema 7.13. Qualquer conjunto semi-algébrico $V \subset \mathbb{R}^{m}$ admite uma estratificação de Whitney, com um número finito de estratos, cada qual semialgébrico.

Demonstração. Assim como mostramos para os conjuntos algébricos, fazemos uma filtração $V=V_{d} \supseteq V_{d-1} \supseteq \ldots$, onde $d=\operatorname{dim}(V)$.

Suponha indutivamente que construímos $V_{i}$, com $V_{i}$ fechado em $V$, de dimensão menor ou igual a $i$. Além disso, suponha que $V_{s+1}-V_{s}$ é uma variedade suave de dimensão $s$, para os $V_{s}$ até então construídos.

Defimos $V_{i-1}=V_{i}$, se $\operatorname{dim}\left(V_{i}\right)<i$. Caso $\operatorname{dim}\left(V_{i}\right)=i$, colocamos

$$
V_{i-1}=\overline{\cup W\left(V_{i}, V_{s}-V_{s-1}\right)} \text {. }
$$

O fecho é tomado em $V$. O objetivo desta construção é eliminar o fecho dos pontos onde os estratos pré-definidos não são Whitney-regulares. Os comentários anteriores mostram que $V_{i-1}$ assim definido é semi-algébrico e $\operatorname{dim}\left(V_{i-1}\right) \leq i-1$. Como estamos essencialmente eliminando um subconjunto fechado de $V_{i}-\sum V_{i}$, temos que $V_{i}-V_{i-1}$ é variedade suave. Por construção, esta estratificação é Whitneyregular. Assim, o resultado segue por indução.

Exemplo 7.14. Considere $G=G L_{n}(\mathbb{C}) \times G L_{n}(\mathbb{C}), M=H o m\left(\mathbb{C}^{n}, \mathbb{C}^{n}\right)$ e a seguinte G-ação sobre $M$ :

$$
(g, h) \cdot u=h \circ u \circ g^{-1} .
$$

Defina $u_{k}\left(e_{i}\right)=e_{i}, i=1, \ldots, k$ e $u_{k}\left(e_{i}\right)=0, i>k$, onde $\left\{e_{i}\right\}, i=1, \ldots, n$, representa a base canônica de $\mathbb{C}^{n}$. 
Temos $G \cdot u_{k}=\{u \in M: \operatorname{rank}(u)=k\}$. Seja $A$ a hipersuperfície $\{u \in M:$ $\operatorname{det}(u)=0\}$.

Então

$$
A=\bigcup_{k=0}^{n-1}\{u \in M: \operatorname{rank}(u)=k\}=\bigcup_{k=0}^{n-1} G \cdot u_{k}
$$

Um resultado importante de [6], Proposição 1.14, garante que, nessas condições, como $A$ é localmente fechado, esta decomposição dá uma estratificação de Whitney de $A$.

Exemplo 7.15. A partição de $\mathbb{R}^{n+1}$, determinada pelo número de zeros complexos de $a_{0} z^{n}+\ldots+a_{n+1}$ e suas multiplicidades, define uma estratificação semi-algébrica de $\mathbb{R}^{n+1}$. 


\section{Capítulo 8}

\section{Classes de Schwartz}

Iniciamos aqui o estudo das classes de Chern para variedades singulares. Neste capítulo, com base no artigo [1], construiremos as classes de Schwartz. A construção, bastante geométrica, utiliza o conceito de tubos e prolongamento de campos de vetores.

A idéia é encontrar um substituto para o fibrado tangente para o caso de uma variedade singular. M.H. Schwartz utiliza a união dos espaços tangentes e seções especiais: as seções radiais.

Aqui sempre consideraremos $X$ uma variedade analítica complexa de dimensão $n$, mergulhada numa variedade analítica complexa suave $M$ de dimensão $m$ e $(\chi)$ uma estratificação de Whitney de $X$ (note que, adicionando o estrato $M-X$, obtemos uma estratificação de Whitney de $M$ ).

Definição 8.1. Um campo estratificado $v$ sobre $A \subseteq M$ é um campo tangente a $M$ tal que, para todo $x \in A, v(x)$ é tangente ao estrato contendo $x$ (denotado por $E(x))$.

Definição 8.2. Um campo radial $v$ em $a \in X$ é um campo estratificado tal que existe um $\varepsilon_{0}>0$ tal que $v$ está saindo de $B(a, \varepsilon)$, para $0<\varepsilon \leq \varepsilon_{0}$.

\subsection{Células duais}

Suponha que $M$ admita uma triangulação $(K)$ e que a mesma seja compatível com a estratificação (todo simplexo aberto está contido num único estrato, o que é possível, por [13]). 
Considere $\left(K^{\prime}\right)$ como sendo a subdivisão baricêntrica de $(K)$. Define-se a triangulação de $M$ dual a $(K)$ (denotada por $(D)$ ), da seguinte forma:

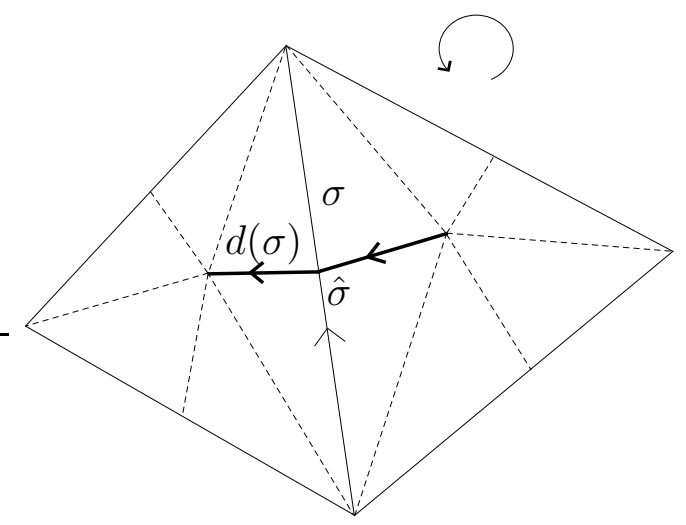

FIGURA 8.1: Células duais

para cada $q$-simplexo $\sigma$ de $(K)$, denote por $d(\sigma)=\left\{\tau \in\left(K^{\prime}\right): \tau \cap \sigma=\hat{\sigma}\right\}$. Temos que $d(\sigma)$ é homeomorfo a uma bola de dimensão real $2 m-q$.

Dada uma orientação para $M$ e para $(K)$ (compatível com a estratificação), é possível orientar $(D)$ de forma que a orientação do simplexo $\sigma$ seguida da orientação de $d(\sigma)$ dê a orientação de $M$.

Existem pelo menos duas grandes vantagens observadas por M.H. Schwartz ao considerarmos a decomposição dual. A primeira é que as células duais são transversais à estratificação de $M$. A segunda é que, se $\sigma$ for um $(r-1)$-simplexo contido num estrato $V_{i}$ de dimensão complexa $k$ (denotado por $V_{i}^{k}$ ), então a dimensão de $d(\sigma) \cap V_{i}^{k}$ vale $2(k-r+1)$, que é a dimensão de obstrução à construção de $r$-seções estratificadas linearmente independentes do pseudo-fibrado $E$ (cuja fibra em cada ponto $x$ é o tangente ao estrato $E(x))$ sobre $V_{i}^{k}$.

Notamos aqui a dificuldade que é estender para o caso singular a idéia do caso suave, visto que para cada estrato a dimensão de obstrução é diferente.

\subsection{Tubos}

\subsubsection{Tubos celulares}

Os elementos desses tubos serão células duais aos simplexos de $X$.

Seja $\sigma$ um $q$-simplexo da triangulação $(K)$ e $y \in \sigma$. O tubo celular de raio $\varepsilon$, denotado por $T_{\varepsilon}(y)$, é construído da seguinte forma. Dado um ponto 
em um simplexo, podemos considerá-lo como combinação linear de seus vértices (coordenadas baricêntricas). Considere os pontos $\bar{y}$ pertencentes aos simplexos $\bar{\sigma}$ tais que $\bar{\sigma}$ possui vértices em comum com $\sigma$ (desta forma as coordenadas baricêntricas de $\bar{y}$ envolvem também os vértices de $\sigma$ ). Estarão no tubo $T_{\varepsilon}(y)$ os pontos cujas coordenadas relativas aos vértices de $\sigma$ são múltiplas das de $y$, com proporção maior ou igual a $1-\varepsilon$.

Se $A \subseteq X$, denotamos por $T_{\varepsilon}(A)$ a reunião $\operatorname{dos} T_{\varepsilon}(y)$, com $y \in A$.

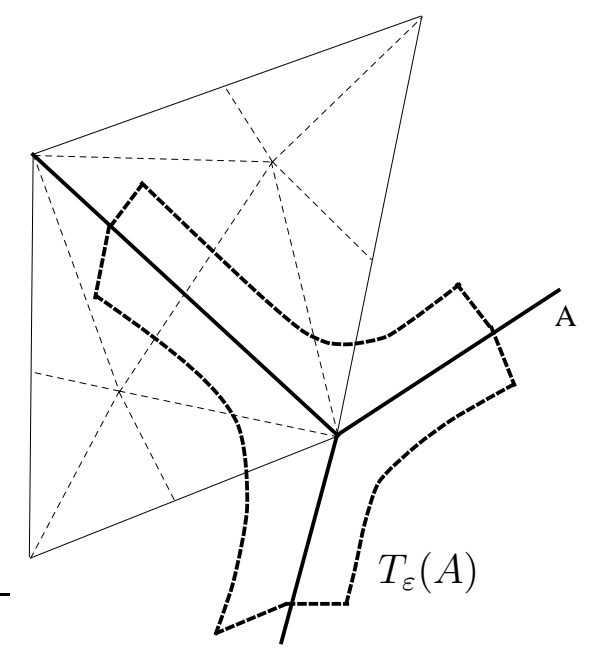

FIGURA 8.2: Tubo celular

\subsubsection{Tubos geodésicos}

Seja $A$ um subconjunto fechado de $V_{i}^{k}$ de tal forma que esteja definida uma métrica riemanniana $\mu$ numa vizinhança de $A$ em $M$. Para todo $y \in A$, considere os arcos geodésicos ortogonais a $A$ em $y$ (vide construção da aplicação Exp na seção 4.3), de comprimento fixado $\mu(y)$. Este conjunto, de dimensão $2(m-k)$, é denotado por $\Theta_{\mu}(y)$. O tubo geodésico $\Theta_{\mu}(A)$ é a reunião $\operatorname{dos} \Theta_{\mu}(y)$, com $y \in A$. Por construção, $\Theta_{\mu}(A)$ é uma vizinhança de $A$ cujos raios $\Theta_{\mu}(y)$ são ortogonais ao estrato $V_{i}^{k}$. 


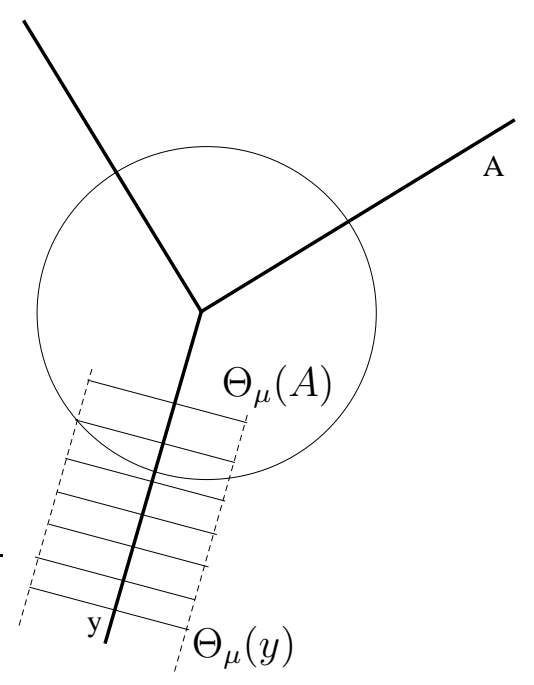

FIGURA 8.3: Tubo geodésico

\subsection{Prolongamentos}

O prolongamento radial local é essencial para a construção das classes de Schwartz. Ele é antecedido por duas etapas de prolongamentos nas quais são utilizadas as condições de regularidade de Whitney. A primeira condição auxilia na construção do prolongamento por paralelismo e a segunda auxilia na construção do prolongamento transversal. O seguinte exemplo ilustra bem como são feitas estas etapas.

Exemplo 8.3. Considere as coordenadas $(x, y) \in \mathbb{C}^{k} \times \mathbb{C}^{n-k}$, em que $\mathbb{C}^{k}$ faz o papel do estrato $V_{i}^{k}$ e $\mathbb{C}^{n}$, o da variedade suave $M$.

Suponha que na bola $B^{k}$ de $\mathbb{C}^{k}$ esteja definido um campo $v$ tangente a $\mathbb{C}^{k}$ com possível singularidade isolada em $\{0\}$. Estendemos este campo por paralelismo a um campo $v^{\prime}$ em $B^{k} \times \mathbb{C}^{n-k}$, ou seja $v^{\prime}(x, y)=v(x)$ (vide figura 8.4).

Agora tomamos o campo transversal definido em $\mathbb{C}^{n}, w(x, y)=(0, y)$, e definimos o prolongamento radial de $v$ como sendo o campo $\tilde{v}=v^{\prime}+w$, definido na bola $B^{n}$ de $\mathbb{C}^{n}$. Note que este campo $\tilde{v}$ possui singularidade isolada na origem se, e somente se, o campo original $v$ possui. Um resultado ([1]) mostra que o índice $I(v,\{0\})$ calculado em $\mathbb{C}^{k}$ tem o mesmo valor de $I(\tilde{v},\{0\})$ calculado em $\mathbb{C}^{n}$.

\subsubsection{Prolongamento por paralelismo}

Construiremos o prolongamento por paralelismo, de um campo definido num estrato $V_{i}^{k}$ (ou em um subconjunto seu), sobre o seu tubo celular. Considere uma 


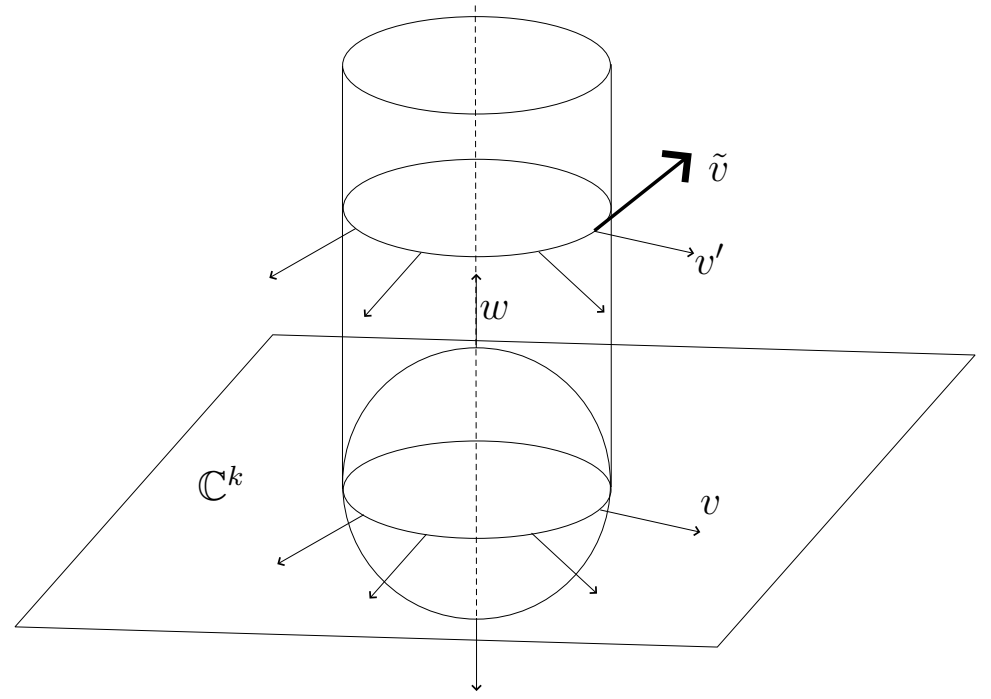

FIGURA 8.4: Campo radial prolongado

decomposição celular de $V_{i}^{k}$ com células $V_{i}^{k} \cap U_{t}$ onde $U_{t}$ são células duais a uma triangulação $(K)$ de $M$ compatível com a estratificação. Seja $\left\{\alpha_{t}\right\}$ partição da unidade associada ao recobrimento de $V_{i}^{k}$ por $\left\{V_{i}^{k} \cap U_{t}\right\}$.

A primeira condição de Whitney permite mostrar que todo vetor $v(y)$ tangente ao estrato de $y$ é prolongável a uma vizinhança de $y$. Isto é, se $y \in U_{t}$, existe uma aplicação contínua $\psi_{t}$ de $U_{t} \times \mathbb{C}^{k}$ no espaço tangente a $U_{t}$, tal que, restrita a $y \times \mathbb{C}^{k}$, é um isomorfismo $\mathbb{C}$-linear sobre o espaço tangente a $y$. Dado $x \in T_{\varepsilon}\left(V_{i}^{k}\right)$, sabe-se que existe um único raio contendo $x$, denotado por $[y, x]$, para algum $y \in U_{t} \cap V_{i}^{k}$. Coloquemos

$$
v^{\prime}(x)=\sum_{t} \alpha_{t}(y) v^{(t)}(x)
$$

onde $v^{(t)}(x)$ é o vetor tal que $\psi_{t}^{-1}(v(y))$ e $\psi_{t}^{-1}\left(v^{(t)}(x)\right)$ têm a mesma projeção em $\mathbb{C}^{k}$.

\subsubsection{Campos transversais}

A construção destes campos utiliza o campo gradiente $G(x)=\nabla(x)$ (gradiente da distância ao estrato $V_{i}^{k}$ ). Inicia-se com o campo gradiente sobre os estratos de dimensão nula e prossegue-se por indução crescente sobre a dimensão dos estratos.

O campo $G$ é tangente aos raios dos tubos geodésicos $\Theta_{\mu}\left(V_{i}^{k}\right)$, mas não é um campo estratificado. Além disso, sua projeção $G^{\prime}$ sobre os estratos não é contínua (vide figura 8.5) 


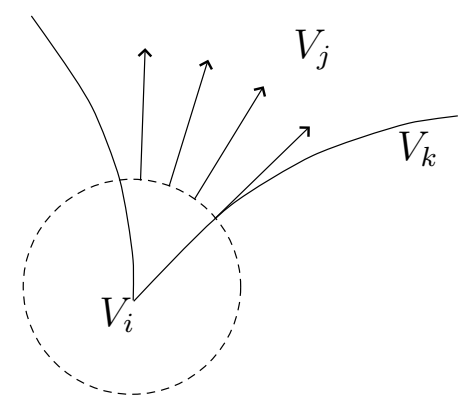

FIGURA 8.5: Campo não-estratificado

Para prolongar o campo $Y^{k}$ definido no fecho de um estrato $\overline{V^{k}}$ para $\overline{V^{j}}$, com $\overline{V^{k}} \subset \overline{V^{j}}$, faremos uma combinação linear entre $G^{\prime}$ e $Y^{k}$, procedendo da seguinte forma:

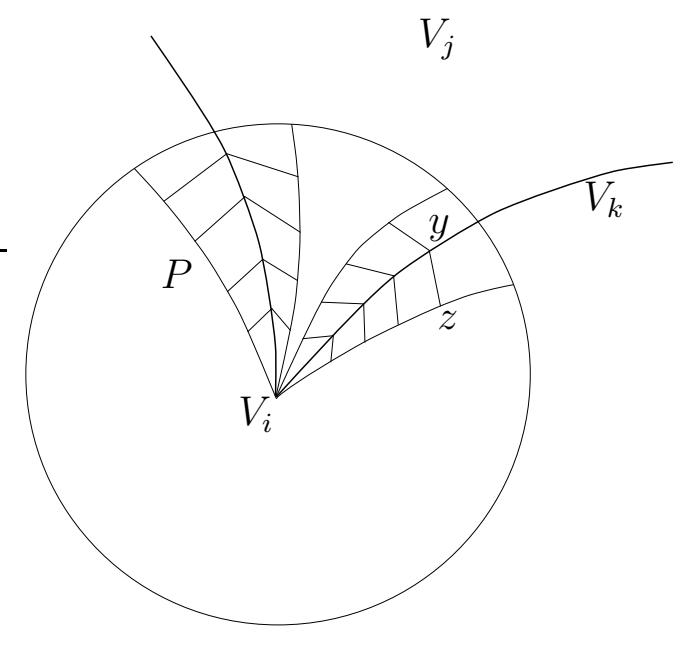

FIGURA 8.6: Vizinhança prismática

Construímos, na vizinhança geodésica $\Theta_{\mu}\left(V^{k}\right)$, um tubo $T_{\varepsilon}\left(V^{k}\right)$, vizinhança de $\overline{V^{k}}$ em $\overline{V^{j}}$, chamada vizinhança prismática. Dado um ponto $x$ nesta vizinhança, sabe-se que existe um único raio $[y, z]$ do tubo celular, contendo $x$ e tal que $y \in V^{k}$ e $z \in V^{j}$. Coloquemos $\lambda=\frac{|\overline{y x}|}{|y z|}$ e assim definimos o novo campo transversal:

$$
Y(x)=(1-\lambda) Y^{k}(y)+\lambda G^{\prime}(x) .
$$

Vemos que se $x=y \in V^{k}$, então $\lambda=0$ e daí o campo $Y$ é o campo original $Y^{k}$. No outro extremo, se $x=z$, então $\lambda=1$ e então o campo $Y$ se torna $G^{\prime}$. Desta forma o campo transversal está estendido numa vizinhança do estrato. 
A condição $b$ da regularidade de Whitney vem mostrar que, se este prolongamento é feito num subconjunto compacto de $V^{k}$, o ângulo entre $Y(x)$ e $G(x)$ é pequeno, o que é importante para o cálculo dos índices a seguir. Utiliza-se a partição da unidade para estender o prolongamento a todo o estrato e o argumento prossegue por indução na dimensão.

\subsubsection{Prolongamento radial local}

Suponha novamente que construímos os tubos geodésicos e celulares de uma parte fechada $A$ de um estrato $V^{k}$ tais que $\Theta_{\mu}(A) \supset T_{\varepsilon}(A)$, de forma que estejamos nas condições acima. Seja $v$ o campo de vetores tangente a $V^{k}$ sobre $A$, com singularidades isoladas $a_{j} \in A$. No tubo celular, definimos o prolongamento por paralelismo $v^{\prime}$ e, como o mesmo se trata de uma vizinhança prismática, definimos o campo transversal $w$. Assim como no Exemplo 8.3, fazemos neste tubo $v^{\prime \prime}=v^{\prime}+w$. Este campo, prolongamento radial local, tem as seguintes propriedades:

(i) $v^{\prime \prime}$ sai ao longo de qualquer tubo $\Theta_{\mu^{\prime}}(A) \subset T_{\varepsilon}(A)$, ao longo de $\partial \Theta_{\mu^{\prime}}(A)-$ $\Theta_{\mu^{\prime}}(\partial A)$

(ii) $\left\{a_{j}\right\}$ também são as singularidades de $v^{\prime \prime}$ e $I\left(v^{\prime \prime}, a_{j}\right)=I\left(v, a_{j}\right)$;

(iii) Fixado o campo transversal $w$, se dois campos iniciais em $A, v_{1}$ e $v_{2}$, são homotópicos, então os correspondentes prolongamentos radiais locais $\left(v_{1}\right)^{\prime \prime}=$ $\left(v_{1}\right)^{\prime}+w$ e $\left(v_{2}\right)^{\prime \prime}=\left(v_{2}\right)^{\prime}+w$ são homotópicos em $T_{\varepsilon}\left(V^{k}\right)$.

\subsection{Construção das classes de Schwartz}

As classes de Schwartz serão construídas através da obstrução determinada por um campo, chamado campo radial, que é uma seção de $E^{r}$, o pseudo-fibrado de $r$ referenciais. Suas propriedades serão apresentadas no teorema a seguir. Ilustraremos a construção do campo, para $r=1$.

Sobre os estratos de dimensão nula $\left\{a_{j}\right\}$, tomamos um campo estratificado radial $v$, saindo de uma bola suficientemente pequena de cada $a_{j}$. A restrição deste campo $v$ aos estratos de dimensão 1 pode ser estendida a todo este estrato, com possíveis singularidades isoladas $a$, com índices $I(v, a)$ (neste exemplo, o índice valeria -1). Este novo campo, por sua vez, pode ser estendido a uma vizinhança celular de cada estrato, pelo método do prolongamento radial local, da seção anterior. Sabemos que o valor do índice $I(v, a)$ continuará o mesmo. Novamente estendemos a restrição deste campo aos estratos de dimensão 2 e este argumento prossegue por 


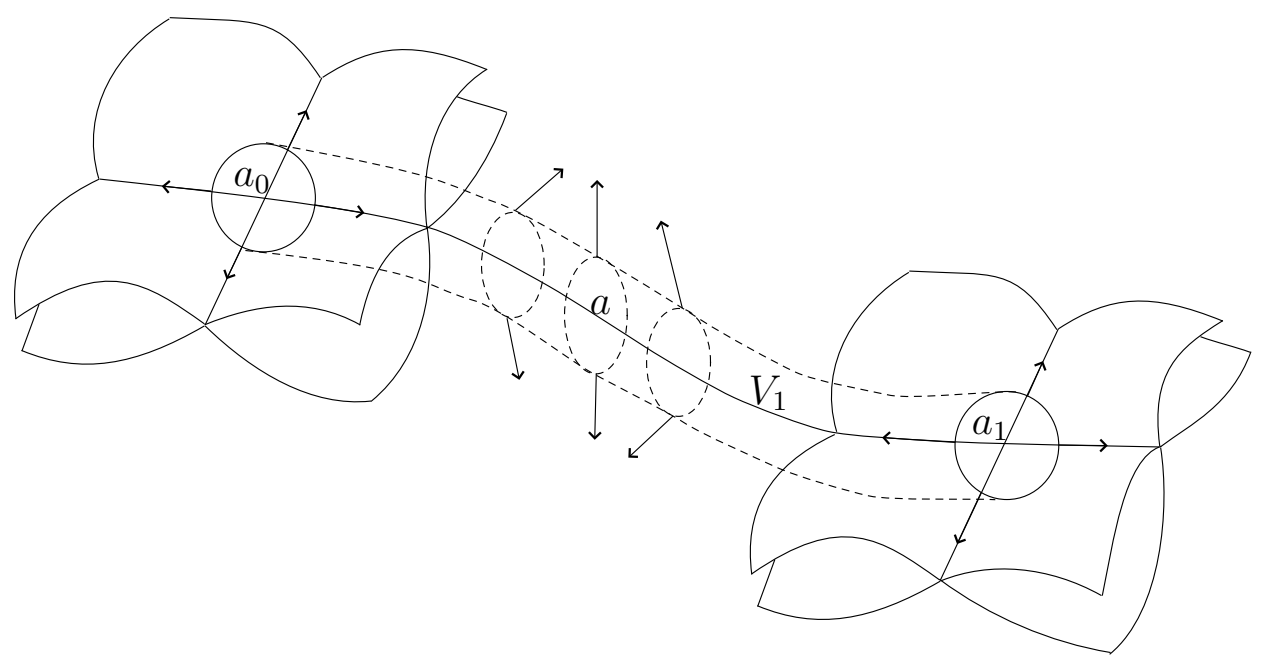

FIGURA 8.7: Construção do campo radial

indução crescente na dimensão dos estratos.

Notamos que a noção de prolongamento radial local pode ser facilmente generalizada para o caso de $r$-referenciais, com as mesmas boas propriedades enunciadas na seção anterior.

Teorema 8.4. Existe uma seção de $E^{r}$, com singularidades isoladas sobre as células duais de dimensão real $2 s=2(m-r+1)$. Esta seção, denominada campo radial $e$ denotada por $v^{r}=\left(v^{r-1}, w\right)$ (separando o último vetor) satisfaz:

(i) As singularidades de $v^{r}$ são isoladas e são zeros de w. Sobre as células de dimensão $2 s-1, v^{r}$ não tem singularidades. Sobre as células de dimensão $2 s, v^{r-1}$ não tem singularidades;

(ii) Se uma célula $d(\sigma)$ de dimensão $2 s$ encontra vários estratos, as singularidades de $v^{r}$ estão no estrato de dimensão menor;

(iii) Se $\{a\} \in d(\sigma) \cap V_{i}^{k}$ é uma singularidade de $v^{r}$ então podemos ter:

- a dimensão de obstrução do estrato $2(k-r+1)$ (que é a dimensão $\left.d(\sigma) \cap V_{i}^{k}\right)$, é igual a zero. Então $I\left(v^{r}, a\right)=+1$.

- $2(k-r+1)>0$ e, neste caso, $I\left(v^{r}, a\right)=I\left(v_{\mid d(\sigma) \cap V_{i}^{k}}^{r}, a\right)$.

Estamos em ponto então de construir as classes de Schwartz. Seja $T_{i}$ a reunião das células duais (fechadas), que interceptam $V_{i}^{k}$. O campo radial $v^{r}$ determina um cociclo de obstrução $\tilde{c}_{i} \in C^{2 s}\left(M, M-i n t\left(T_{i}\right)\right)$ sobre o espaço das cocadeias duais de dimensão $2 s$ tal que a avaliação vale: 


$$
<\tilde{c}_{i}, d^{s}>=\sum_{a_{j} \in V_{i}^{k} \cap d^{s}} I\left(v^{r}, a_{j}\right) .
$$

A soma é feita sobre as singularidades $\left\{a_{j}\right\}$, que estão nas células de dimensão complexa $s$. Enfatizamos que a dimensão da interseção no somatório é justamente $2(k-r+1)$, que é a dimensão de obstrução à construção de $r$-referenciais em $V_{i}^{k}$. Esta tentativa nos remonta à construção das classes de Chern por obstrução no caso suave e, como dissemos, é uma vantagem ao se considerar a construção do campo radial através das células duais.

Seja $T$ a reunião das células duais (fechadas) que encontram $X$. Considere $\tilde{c}_{i}$ como elemento de $C^{2 s}(M, M-i n t(T))$ (pelo homomorfismo restrição) e coloque $\tilde{c}=\sum_{i} \tilde{c}_{i}$.

A classe deste elemento em $H^{2 s}(M, M-X)$ é a classe de Schwartz de grau $s$ de $X$ e denotada por $c^{s}(X)$. Em [1], mostra-se que esta classe independe da triangulação e da estratificação consideradas. 


\section{Capítulo 9}

\section{Obstrução de Euler e classes de MacPherson}

Assim como no capítulo anterior, estamos interessados em construir classes de Chern para variedades singulares, porém, com uma abordagem diferente, menos geométrica e mais axiomática. A existência destas classes (agora em homologia) foi conjecturada por Deligne e Grothendieck para variedades algébricas, e provada por R. MacPherson em 1974. No entanto, em 1981, Brasselet e Schwartz ([1]) mostraram a existência de um isomorfismo (isomorfismo de Alexander) entre as classes de Schwartz e as de MacPherson.

A referência deste capítulo é o artigo [15].

\subsection{Conjectura de Deligne-Grothendieck}

Para compreendermos o que diz a conjectura de Deligne-Grothendieck, necessitamos definir conjuntos e funções construtíveis.

Definição 9.1. Um conjunto construtivel em uma variedade algébrica $V$ é aquele que pode ser obtido de subvariedades algébricas de $V$ através de um número finito de operações de união e complementação.

Definição 9.2. Uma função construtivel $\alpha: V \rightarrow \mathbf{Z}$ é aquela em que $V$ admite uma partição finita em subconjuntos construtíveis tais que $\alpha$ é constante sobre cada um deles. Assim, se $Y_{i}, i=1, \ldots, n$, são os tais subconjuntos construtíveis de $V$, e $1_{\mid Y_{i}}$ é a função característica de $Y_{i}$, então $\alpha$ pode ser escrita como:

$$
\alpha=\sum_{i=1}^{n} \alpha_{i} 1_{\mid Y_{i}},
$$

onde $\alpha_{i} \in \mathbf{Z}, i=1, . ., n$, são os valores assumidos pela função $\alpha$. 
As funções construtíveis formam um grupo abeliano com a soma.

Proposição 9.3 (Proposição 1). Existe um único funtor covariante $F$ da categoria das variedades algébricas compactas na categoria dos grupos abelianos tal que $F(V)$ é o grupo das funções construtíveis em $V$ e a cada a cada morfismo $f: V \rightarrow W$ está associado o homomorfismo $f_{*}: F(V) \rightarrow F(W)$ tal que, para todo fechado $Y$ de $V$,

$$
f_{*}\left(1_{\mid Y}\right)(w)=\chi\left(f^{-1}(w) \cap Y\right) .
$$

A demonstração pode ser encontrada na referência citada. Nela, dada $\alpha \in$ $F(V)$, define-se $f_{*}(\alpha) \in F(W)$ da seguinte forma. Suponha $\left\{S_{j}\right\}$ uma estratificação de $V$ subordinada a $\alpha$ e a $f$. Coloque:

$$
f_{*}(\alpha)(w)=\sum_{j} \alpha\left(S_{j}\right) \chi\left(f^{-1}(w) \cap S_{j}\right)
$$

e daí o resultado segue das boas propriedades da característica de Euler em cohomologia com suporte compacto.

Teorema 9.4 (Conjectura de Deligne-Grothendieck). Dada qualquer variedade algébrica compacta $V$, para toda função construtivel $\alpha$ em $V$ existe uma única classe $c_{*}(\alpha) \in H_{*}(V)$ satisfazendo:

(i) $c_{*}(\alpha+\beta)=c_{*}(\alpha)+c_{*}(\beta)$;

(ii) Para todo morfismo $f: V \rightarrow W$, temos $f_{*}\left[c_{*}(\alpha)\right]=c_{*}\left(f_{*}(\alpha)\right)$, onde, no primeiro lado da igualdade, $f_{*}$ indica o homomorfismo induzido por $f$ em homologia;

(iii) Se $V$ for uma variedade suave, então $c_{*}\left(1_{\mid V}\right)=c(V) \cap[V]=$ dual $c(V)$ (isomorfismo de Poincaré).

A partir desta conjectura, Deligne definiu a classe total de Chern de uma variedade compacta $V$ como sendo $c_{*}\left(1_{\mid V}\right)$.

A hipótese de compacidade pode ser retirada considerando-se aplicações próprias e homologia com suporte localmente finito.

Através destas boas propriedades, podemos obter um resultado útil para classes de Chern, no caso suave:

Corolário 9.5. Sejam $V$ e $X_{i}, i=1, \ldots, n$ variedades algébricas suaves. Suponha que existam $k_{i} \in \mathbf{Z}$ e $g_{i}: X_{i} \rightarrow V$ tais que, para qualquer $p \in V, \sum_{i} k_{i} \chi\left(g_{i}^{-1}(p)\right)=1$. Então dual $c(V)=\sum_{i} k_{i}\left(g_{i}\right)_{*}\left[\right.$ dual $\left.c\left(X_{i}\right)\right]$.

Demonstração. Pela propriedade (iii) da conjectura, temos 


$$
\sum_{i} k_{i}\left(g_{i}\right)_{*}\left[\text { dual } c\left(X_{i}\right)\right]=\sum_{i} k_{i}\left(g_{i}\right)_{*}\left[c_{*}\left(1_{\mid X_{i}}\right)\right]
$$

Agora aplicando a propriedade da naturalidade, (ii), segue que

$$
\sum_{i} k_{i}\left(g_{i}\right)_{*}\left[c_{*}\left(1_{\mid X_{i}}\right)\right]=\sum_{i} k_{i} c_{*}\left[\left(g_{i}\right)_{*}\left(1_{\mid X_{i}}\right)\right] .
$$

Pela Proposição 9.3,

$$
\sum_{i} k_{i} c_{*}\left[\left(g_{i}\right)_{*}\left(1_{\mid X_{i}}\right)\right]=\sum_{i} k_{i} c_{*}\left[\chi g_{i}^{-1}(\cdot)\right] .
$$

Finalmente, usando a linearidade das classes, e a hipótese do corolário, segue o resultado:

$$
\sum_{i} k_{i} c_{*}\left[\chi g_{i}^{-1}(\cdot)\right]=c_{*}\left[\sum_{i} k_{i} \chi g_{i}^{-1}(\cdot)\right]=c_{*}\left(1_{\mid V}\right)=\operatorname{dual} c(V)
$$

\subsection{A obstrução local de Euler}

MacPherson encontrou uma fórmula algébrica para as classes do Teorema 9.4. e esta utiliza o conceito de obstrução de Euler. Daremos aqui duas construções desta obstrução: a primeira, do próprio MacPherson, e uma segunda, mais geométrica, de Brasselet e M.H. Schwartz. Para ambas, definiremos o fibrado de Nash.

Aqui, $X$ é uma variedade analítica de dimensão $n$, que admite estratificação de Whitney, mergulhada numa variedade analítica suave $M$. Sobre $M$ está definido um fibrado $\tilde{M}$ cuja fibra em $x \in M$ é o conjunto $G_{n}\left(T_{x} M\right) \simeq G_{n}\left(\mathbb{C}^{m}\right)$. Denote por $\nu: \tilde{M} \rightarrow M$ a projeção deste fibrado.

Sobre a parte regular $X_{\text {reg }}$ de $X$, existe uma seção $s$ deste fibrado, dada por $s(x)=T_{x}\left(X_{r e g}\right)$, já que $T_{x}\left(X_{r e g}\right)$ consiste de um $n$-plano que é subespaço de $T_{x} M$.

A modificação de Nash $\tilde{X}$ de $X$ é o fecho em $\tilde{M}$ de $\operatorname{Im}(s)$. A projeção $\nu$ induzida pela restrição a $\tilde{X}$ (e ainda denotada por $\nu$ ) também é analítica. Na figura a seguir, representamos a transformação de Nash de um cone. Note que $\nu$, sobre $X_{r e g}$, é um homeomorfismo. Cada ponto de $\tilde{X}$ é um $n$-plano, e cada ponto de $\nu^{-1}(0)$ é limite de uma seqüência de planos tangentes a pontos convergindo a 0 .

Denotaremos por $\tilde{E}$ o fibrado de Nash sobre $\tilde{X}$, que é a restrição a $\tilde{X}$ do fibrado tautológico sobre $\tilde{M}$ (a fibra de cada plano é o conjunto de vetores que constituem este plano). 


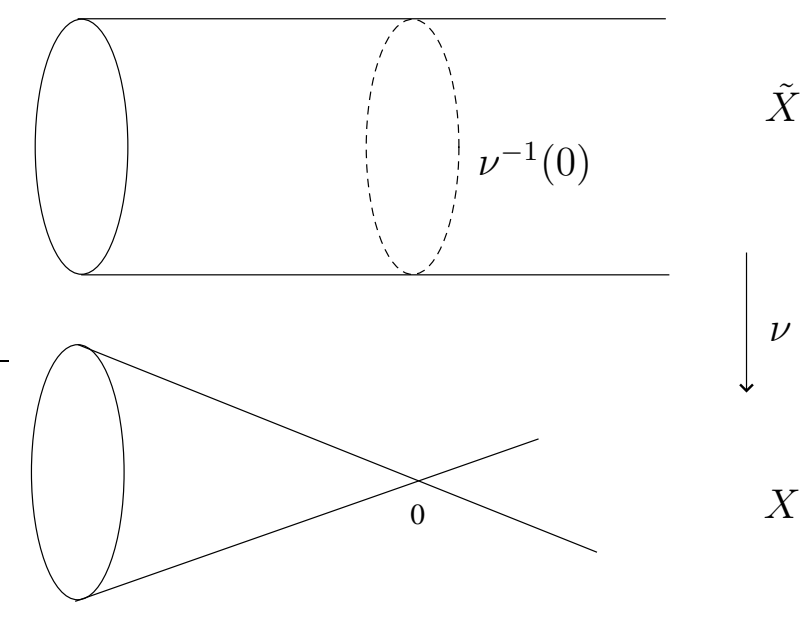

FIGURA 9.1: Modificação de Nash

Podemos vizualizar $\tilde{E}$ da seguinte forma. Coloque $\Lambda$ como sendo o fibrado sobre $M$, produto de $T M(\operatorname{com}$ projeção $\pi)$ e $\tilde{M}(\operatorname{com}$ projeção $\nu)$. Então:

$$
\tilde{E}=\{(v, \tilde{x}) \in T M \times \tilde{M}: \pi(x)=\nu(\tilde{x})=x \text { e } v(x) \in \tilde{x}\} .
$$

Denote por $\nu_{*}: \tilde{E} \rightarrow T M_{\mid X}$ a restrição da projeção $\Lambda \rightarrow T M$ a $\tilde{E}$. Podemos enunciar o seguinte resultado, de [3], sobre levantamento de seções do pseudo-fibrado $E$ (isto é, um campo estratificado) ao fibrado vetorial $\tilde{E}$.

Proposição 9.6. Seja $A$ um subconjunto de $X$ onde está definida uma seção $v$ de E. Então existe um único levantamento $\tilde{v}$ de $v$ como seção de $\tilde{E}$ sobre $\nu^{-1}(A)$.

Demonstração. Devemos mostrar que, se $x \in A$, para todo ponto $\tilde{x}$ de $\nu^{-1}(x)$, existe um único vetor $\tilde{v}(\tilde{x}) \in \tilde{E}(\tilde{x})$ tal que $\nu_{*}(\tilde{v}(\tilde{x}))=v(x)$.

Suponha inicialmente que $x$ é um ponto regular de $X$. Então $\nu^{-1}(x)=\tilde{x}=$ $T_{x}\left(X_{\text {reg }}\right)$. Como $v(x) \in T_{x}\left(X_{\text {reg }}\right)$, pela última caracterização de $\tilde{E}$, podemos definir $\tilde{v}(\tilde{x})=(v(x), \tilde{x})$.

Agora suponha que $x$ é um ponto singular, pertencente ao estrato $V_{i}$ da estratificação de Whitney de $X$. Por definição da modificação de Nash, existe uma seqüência de pontos $\tilde{x_{n}} \in \operatorname{Im}(s)$ convergindo a $\tilde{x}$. Colocando $\tilde{x_{n}}=T_{x_{n}}\left(X_{\text {reg }}\right)$, com $x_{n} \in X_{\text {reg }}$, temos

$$
\lim \left(T_{x_{n}}\left(X_{\text {reg }}\right)\right)=\lim \left(\tilde{x_{n}}\right)=\tilde{x} .
$$

Pela condição (a) da regularidade de Whitney, segue que $T_{x}\left(V_{i}\right) \subset \tilde{x}$. Então temos que $v(x) \in \tilde{x}$ e podemos novamente definir $\tilde{v}(\tilde{x})=(v(x), \tilde{x})$. 
Passemos agora à construção da obstrução local de Euler. Seja $p \in X$. Numa vizinhança de $p$ em $M$, considere as coordenadas locais $z=\left(z_{1}, \ldots, z_{n}\right)$ tais que que $z(p)=0$. Considere também a função a valores reais

$$
\lambda(z)=\|z\|^{2}=z_{1} \overline{z_{1}}+\ldots+z_{n} \overline{z_{n}} .
$$

Sabemos que a diferencial $d \lambda$ é tal que

$$
d_{p^{\prime}} \lambda: T_{p^{\prime}} M \rightarrow \mathbb{R}
$$

é uma transformação linear, para todo $p^{\prime}$ suficientemente próximo de $p$. Assim, $d_{p^{\prime}} \lambda \in\left(T_{p^{\prime}} M\right)^{*}$ e então $d \lambda$ é uma seção local de $(T M)^{*}$. O seguinte diagrama ilustra a seção $d \lambda$

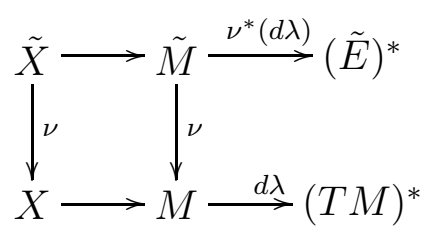

Podemos realizar o pull-back de $d \lambda$ e obter uma nova seção local, agora do dual do fibrado tautológico sobre $M$, que será denotada por $\nu^{*}(d \lambda)$. Para isto, considere $x \in M$ suficientemente próximo de $p$ e $\tilde{x}$ tal que $\nu(\tilde{x})=x$. Como $\tilde{x}$ é um subespaço de $T_{x} M$, definimos

$$
\nu^{*}(d \lambda)(\tilde{x}) \doteq(d \lambda)(\nu(\tilde{x}))_{\mid \tilde{x}}=(d \lambda)(x)_{\mid \tilde{x}}: \tilde{x} \rightarrow \mathbb{R} \in(\tilde{x})^{*}
$$

Note que $(\tilde{x})^{*}$ é uma fibra do dual do fibrado tautológico, assim, restringindo $\nu^{*}(d \lambda)$ a $\tilde{X}$, teremos uma seção local de $(\tilde{E})^{*}$, denotada por $r$. Se $\varepsilon$ é pequeno, esta seção é não-nula em $\nu^{-1}\left(S_{\varepsilon}\right)$. Logo, existe um cociclo de obstrução

$$
E u(\tilde{E}, r) \in H^{2 n}\left(\nu^{-1}\left(B_{\varepsilon}\right), \nu^{-1}\left(S_{\varepsilon}\right), \mathbf{Z}\right)
$$

que, avaliado na classe fundamental de homologia do par $\left[\nu^{-1}\left(B_{\varepsilon}\right), \nu^{-1}\left(S_{\varepsilon}\right)\right]$, dá a obstrução de Euler local

$$
E u_{p}(X)=<E u(\tilde{E}, r),\left[\nu^{-1}\left(B_{\varepsilon}\right), \nu^{-1}\left(S_{\varepsilon}\right)\right]>\in \mathbf{Z} .
$$

\subsubsection{Outra forma de construção}

Suponha que o ponto $p$ pertença ao estrato $V_{i}$ de dimensão $k$ da estratificação de Whitney de $X$. Como o cálculo é feito localmente, podemos considerar uma vizinhança de $p$ em $M$ como sendo um aberto de $\mathbb{C}^{m}$, com origem em $p$ e $V_{i}$ como 
aberto de $\mathbb{C}^{k}$ constituído das $k$-primeiras coordenadas de $\mathbb{C}^{m}$. Denote por $b^{2 k}$ a bola euclideana de centro em $p$ e $\Theta$ o tubo geodésico de $b^{2 k}$.

Seja também $v^{\prime}$ um campo de vetores tangentes a $V_{i}$, saindo de $b^{2 k}$, não nulo em $b^{2 k}-\{p\}$. Construa o prolongamento radial local de $v^{\prime}$ e denote por $v=v_{\mid \partial \Theta}^{\prime}$ (vide figura a seguir). Conforme a Proposição 9.6, este campo pode ser levantado a $\tilde{v}$, uma seção de $\tilde{E}$ sem singularidades sobre $\nu^{-1}(\partial \Theta)$.

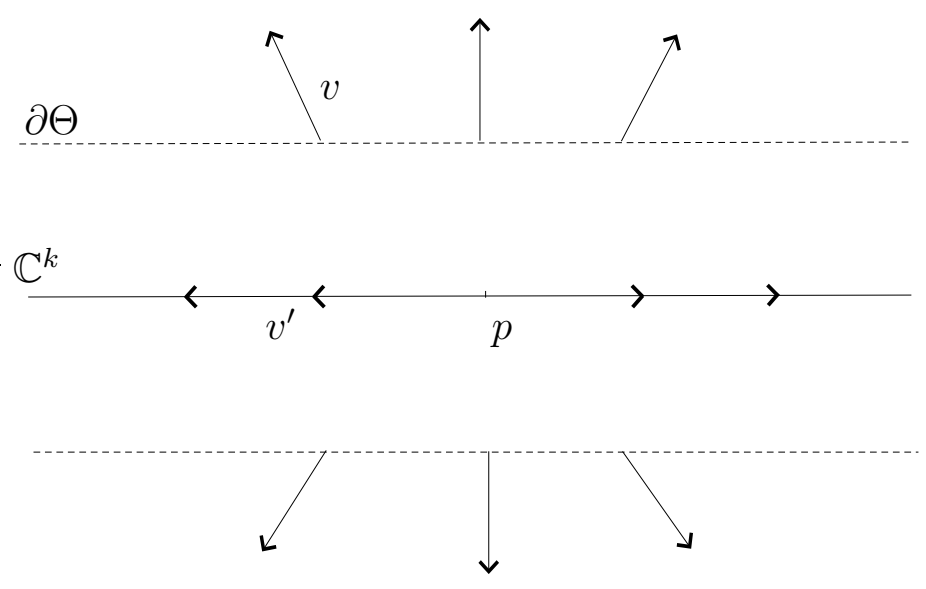

FIGURA 9.2: Obstrução à extensão de $\tilde{v}$ sobre $\nu^{-1}(\Theta)$

A obstrução à extensão de $\tilde{v}$ a uma seção não nula de $\tilde{E}$ sobre $\nu^{-1}(\Theta)$ é a obstrução local de Euler de $X$ em $p$, também denotada por $\operatorname{Obs}\left(\tilde{v}, \tilde{E}^{*}, \nu^{-1}(\Theta)\right)$.

Esta definição independe das vizinhanças tomadas e dos tubos. Listamos aqui as propriedades básicas da obstrução local de Euler ([1]):

(i) É constante ao longo de cada estrato de uma estratificação de Whitney de $X$ (denotaremos por $E u_{V}(X)$ a obstrução calculada em cada ponto de um estrato $V)$;

(ii) $E u_{p}(X)=1$, se $p$ é um ponto não singular;

(iii) $E u_{p \times p^{\prime}}\left(X \times X^{\prime}\right)=E u_{p}(X) \cdot E u_{p^{\prime}}\left(X^{\prime}\right)$.

\subsection{Classe de Mather}

Definição 9.7. A classe de Mather de uma variedade analítica complexa $X$ é definida como a classe em homologia $c_{M}(X)=\nu_{*}(c(\tilde{E}) \cap[\tilde{X}])$, onde $\nu_{*}$ é a induzinda em homologia da projeção $\nu: \tilde{X} \rightarrow X$.

Observe que o produto cap acima está bem definido. Notamos a importância de considerar o fibrado de Nash na tentativa de construção de classes características 
de variedades singulares, como substituto ao fibrado tangente.

Aqui poderíamos naturalmente definir a classe de Mather de uma função construtível $\alpha=\sum_{i=1}^{n} \alpha_{i} 1_{\mid Y_{i}}$ como sendo $c_{M}(\alpha)=\sum_{i=1}^{n} \alpha_{i} c_{M}\left(V_{i}\right)$ e nos perguntar se ela satisfaz os axiomas da conjectura de Deligne-Grothendieck. A resposta é negativa: de acordo com [8], a condição (ii) não é verificada. No entanto, a classe de Mather é um ingrediente fundamental na definição dada por Macpherson. Para isso, necessitamos ainda do conceito de ciclos algébricos.

Definição 9.8. Um ciclo algébrico numa variedade $V$ é uma soma formal finita $\sum n_{i} V_{i}, n_{i} \in \mathbf{Z}$, com $V_{i}$ sendo subvariedades irredutíveis de $V$. A classe de Mather de um ciclo algébrico será $c_{M}\left(\sum n_{i} V_{i}\right)=\sum n_{i} c_{M}\left(V_{i}\right)$ (observando $c_{M}\left(V_{i}\right)$ como elemento de $H_{*}(V)$ pelo homomorfismo inclusão).

MacPherson, na referência citada, define um isomorfismo $T$ do conjunto dos ciclos algébricos de $V$ em $F(V)$, da seguinte forma:

$$
T\left(\sum_{i} n_{i} V_{i}\right)(p)=\sum_{i} n_{i} E u_{p}\left(V_{i}\right)
$$

para todo $p \in V$. É mostrado que $c_{M} \circ T^{-1}$ satisfaz as propriedades da Conjectura de Deligne-Grothendieck e, portanto nos dá uma fórmula explícita para as classes conjecturadas.

As condições (i) e (iii) são de fácil verificação. Já a condição (ii) é mais delicada. Pode-se, no entanto, transformar este problema em outro, e isto é feito da seguinte forma. Vamos mostrar que basta mostrar a condição no caso em que $V$ é suave e $\alpha$ é identicamente 1 .

Para isso, seja $f: V \rightarrow W$ e $\alpha: V \rightarrow \mathbf{Z}$ uma função construtível. Suponha que esta hipótese seja verdadeira.

Existem ([15], Proposição 2) inteiros $k_{i}$ e aplicações $g_{i}: V_{i} \rightarrow V$ com $V_{i}$ suaves tais que $\alpha$ pode ser escrita como $\alpha=\sum_{i} k_{i}\left(g_{i}\right)_{*}(1)$.

Temos então:

$$
f_{*}\left(c_{M} T^{-1}(\alpha)\right)=\sum_{i} k_{i} f_{*}\left(c_{M} T^{-1}\left(g_{i}\right)_{*}(1)\right) .
$$

Aplicando nossa hipótese duas vezes, segue que a última parcela é igual a

$$
\sum_{i} k_{i} c_{M}\left(T^{-1}\left(f \circ g_{i}\right)_{*}(1)\right)=c_{M}\left(T^{-1} f_{*}(\alpha)\right),
$$

que é o que queríamos demonstrar. Assim, a condição (ii) fica satisfeita ao mostrarmos que, para $V$ suave, 


$$
f_{*}(\text { dual } c(V))=c_{M}\left(T^{-1} f_{*}(1)\right) .
$$

O problema então se reduz a encontrar um ciclo algébrico $\sum_{i} n_{i} W_{i}$ em $W$ tal que $T\left(\sum_{i} n_{i} W_{i}\right)=f_{*}(1)$, isto é, usando a Proposição 9.3:

$$
\chi\left(f^{-1}(p)\right)=\sum_{i} n_{i} E u_{p}\left(W_{i}\right)
$$

$\mathrm{e}$

$$
f_{*}(\text { dual } c(V))=\sum_{i} n_{i} c_{M}\left(W_{i}\right) .
$$

A determinação deste ciclo satisfazendo estas duas condições é feita na referência utilizando-se métodos totalmente diferentes dos apresentados aqui. O que enfatizamos na redução deste problema é a aparição da obstrução de Euler nos cálculos e a forma como ela se relaciona com a característica de Euler-Poincaré.

\subsection{Cálculos de obstruções}

Nesta seção, iremos calcular a obstrução de Euler de algumas variedades singulares, usando as propriedades já enunciadas e a seguinte fórmula do tipo Lefschetz, de [2], que vê a obstrução como função construtível:

Teorema 9.9. Seja $V$ uma variedade analitica complexa com singularidade na origem, mergulhada numa variedade suave $M$ e $\left\{V_{\alpha}\right\}$ uma estratificação de Whitney de $V$. Seja $l: U \rightarrow \mathbb{C}$ uma projeção linear genérica, onde $U$ é uma vizinhança aberta da origem em $M$. Então,

$$
E u_{0}(V)=\sum_{\alpha} \chi\left(V_{\alpha} \cap B_{\varepsilon} \cap l^{-1}\left(t_{0}\right)\right) \cdot E u_{V_{\alpha}}(V)
$$

onde $\varepsilon$ é suficientemente pequeno e $t_{0} \in \mathbb{C}-\{0\}$ está perto da origem.

Uma aplicação linear genérica nas condições do teorema é tal que, se $V_{\alpha}$ é um estrato onde $0 \in \bar{V}_{\alpha}$ e $x_{n}$ é uma seqüência em $V_{\alpha}, \operatorname{com} x_{n} \rightarrow 0$ e $T_{x_{n}}\left(V_{\alpha}\right) \rightarrow T$, então o $\operatorname{rank}$ de $d_{0} l_{\mid T}$ é máximo.

Exemplo 9.10. David Mond, em [20], classificou uma classe especial de superfícies singulares $V \subset \mathbb{C}^{3}$ que admitem parametrização analítica $f: U \subset \mathbb{C}^{2} \rightarrow V$. Neste exemplo, analisaremos a família $S_{k}$ :

$$
f: \mathbb{C}^{2} \rightarrow \mathbb{C}^{3}
$$




$$
(X, Y) \mapsto\left(X, Y^{2}, Y^{3}+X^{k+1} Y\right)=(x, y, z)
$$

Implicitamente, sua equação é

$$
2 x^{k+1} y^{2}+y^{3}-z^{2}+x^{2(k+1)} y=0 .
$$

Quando $k=1$, temos a figura a seguir. Se $x=0$, temos a cúspide $y^{3}-z^{2}=0$. Assim, teremos uma família de curvas planas com parâmetro $x$.

As singularidades de $V$ serão os pontos da forma $\left(x,-x^{k+1}, 0\right)$.

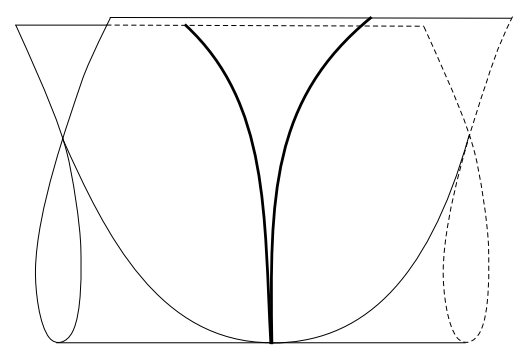

FIGURA 9.3: Membro da família $S_{k}$

Considere a estratificação de Whitney: $V_{0}=\{0\}, V_{1}$ como sendo o conjunto singular, menos a origem, e $V_{2}$ como sendo a parte regular. Considere também a aplicação linear genérica $l(x, y, z)=x$. Aplicando a fórmula do Teorema 9.9, temos:

$$
\begin{aligned}
E u_{0}(V) & =\chi\left(V_{0} \cap B_{\varepsilon} \cap l^{-1}\left(t_{0}\right)\right) \cdot E u_{V_{0}}(V) \\
& +\chi\left(V_{1} \cap B_{\varepsilon} \cap l^{-1}\left(t_{0}\right)\right) \cdot E u_{V_{1}}(V) \\
& +\chi\left(V_{2} \cap B_{\varepsilon} \cap l^{-1}\left(t_{0}\right)\right) \cdot E u_{V_{2}}(V) .
\end{aligned}
$$

Inicialmente note que $V_{0} \cap B_{\varepsilon} \cap l^{-1}\left(t_{0}\right)=\emptyset$, então $\chi\left(V_{0} \cap B_{\varepsilon} \cap l^{-1}\left(t_{0}\right)\right)=0$.

Temos também que $V_{1} \cap B_{\varepsilon} \cap l^{-1}\left(t_{0}\right)$ consiste de apenas um ponto, logo $\chi\left(V_{1} \cap B_{\varepsilon} \cap l^{-1}\left(t_{0}\right)\right)=1$.

Devemos calcular $E u_{V_{1}}(V)$. Para isso, como a obstrução é constante ao longo do estrato, calcularemos $E u_{p}(V)$, para $p \in V_{1}$. Uma vizinhança de $p$ será da forma $V_{1} \times C$, onde $C$ é uma curva (um nó). Como a obstrução é local, $E u_{p}(V)=$ $E u_{p}\left(V_{1} \times C\right)$ e assim podemos usar as propriedades da obstrução já citadas,

$$
E u_{p}(V)=E u_{p}\left(V_{1} \times C\right)=E u_{p}\left(V_{1}\right) \cdot E u_{p}(C)=1 \cdot E u_{p}(C) .
$$

Ao longo de uma curva, a obstrução vale a multiplicidade do ponto na mesma (vide [8]), logo 


$$
E u_{p}(V)=E u_{p}(C)=2 .
$$

Agora resta calcular apenas a última parcela e, para isto, notemos inicialmente que na parte regular temos $E u_{V_{2}}(V)=1$.

Devemos calcular a característica de Euler-Poincaré da curva plana $V_{2} \cap B_{\varepsilon} \cap$ $l^{-1}\left(t_{0}\right)$. Para este cálculo, usaremos a fórmula encontrada no Teorema 2 de [11] que diz que esta característica vale a diferença entre o número de Milnor da fibra genérica $x=x_{0} \neq 0$ (que será número de pontos de Morse sobre a curva - no caso, vale 1 ) e o número de Milnor da curva $x=0$ (que, por definição, vale 2). Logo $\chi\left(V_{2} \cap B_{\varepsilon} \cap l^{-1}\left(t_{0}\right)\right)=-1$.

Finalmente, a obstrução valerá:

$$
E u_{0}(V)=0+1.2+(-1) \cdot 1=1 .
$$

Isto mostra que a obstrução de Euler não distingue os elementos da família $S_{k}$.

Exemplo 9.11. Neste exemplo, analisaremos a família $B_{k}$ :

$$
\begin{gathered}
f: \mathbb{C}^{2} \rightarrow \mathbb{C}^{3} \\
(X, Y) \mapsto\left(X, Y^{2}, X^{2} Y+Y^{2 k+1}\right) .
\end{gathered}
$$

Implicitamente, sua equação é

$$
x^{4} y+2 x^{2} y^{k+1}+y^{2 k+1}-z^{2}=0 .
$$

Se $x=0$, temos a curva $y^{2 k+1}-z^{2}=0$ (novamente, teremos uma família de curvas planas com parâmetro $x$ ).

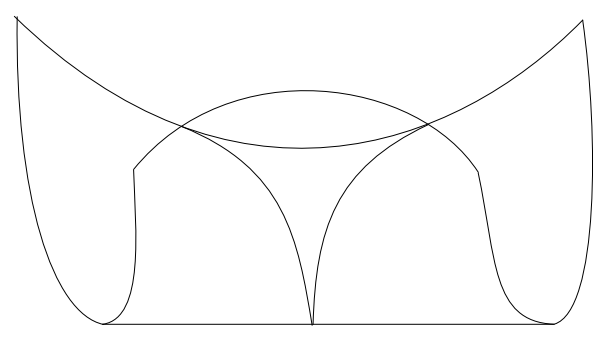

FIGURA 9.4: Membro da família $B_{k}$ 
Os cálculos procedem de maneira análoga ao do exemplo anterior. A única diferença estará no cálculo de $\chi\left(V_{2} \cap B_{\varepsilon} \cap l^{-1}\left(t_{0}\right)\right)$.

Coloque $g(y, z)=y^{2 k+1}-z^{2}$. O número de Milnor da curva $x=0$ vale $\operatorname{dim}_{\mathbb{C}} \frac{\mathcal{O}_{2}}{\left\langle y^{2 k}, z>\right.}=2 k$ (onde $\mathcal{O}_{2}$ indica o anel das séries de potências em 0 e $<y^{2 k}, z>$ é o ideal jacobiano de $g$, isto é, o ideal gerado pelas derivadas parciais de $g$ ).

Já o número de Milnor da fibra genérica continua valendo 1. Assim, $\chi\left(V_{2} \cap\right.$ $\left.B_{\varepsilon} \cap l^{-1}\left(t_{0}\right)\right)=1-2 k$ e daí

$$
E u_{0}(V)=0+1.2+(1-2 k) \cdot 1=3-2 k .
$$

Ou seja, a obstrução de Euler distingue os elementos da família $B_{k}$. 


\section{Capítulo 10}

\section{O teorema de Poincaré-Hopf: versão singular}

No caso singular, o teorema de Poicaré-Hopf não é mais verdadeiro. Mesmo a definição de índice não faz mais sentido, já que necessitamos de uma vizinhança de $a$ homeomorfa a uma bola $B(a)$.

Como exemplo, analisaremos o toro pinçado $X$ definido no Capítulo 2, cuja característica de Euler-Poincaré vale 1. Considere um campo $v$ definido numa vizinhança $B(a) \cap X$ de $a$ em tal que $v(x)$ seja tangente a $X$ para todo $x$ diferente de $a$ e tenha singularidade isolada em $a$. As figuras a seguir ilustram dois campos, sendo o segundo um campo radial:
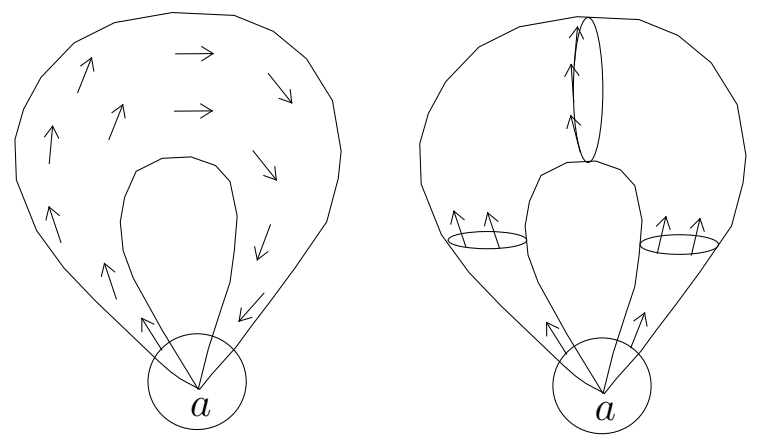

FIGURA 10.1: Campos no toro pinçado

Analisando esses exemplos:

- no primeiro, temos que o campo definido em $\partial B(a) \cap X$ é restrição de um campo definido em $\partial B(a)$ de índice zero, logo o teorema não se verificaria.

- no campo radial, o campo em $\partial B(a) \cap X$ pode ser estendido a um campo de índice 1 , o que verificaria o teorema. 
Isto nos leva a considerar campos radiais na tentativa recuperar o teorema de Poincaré-Hopf.

Observamos inicialmente o campo radial que define as classes de Schwartz, feito na seção 8.4. Por construção, (Figura 8.7) a soma dos índices dá justamente a característica de Euler-Poincaré da variedade singular.

Para campos mais gerais, podemos afirmar que o resultado pode ser estendido para uma variedade $X$, com um número finito de singularidades isoladas $\left\{a_{j}\right\}$, mergulhada numa variedade suave.

Devemos, para isto, considerar um campo $v$, radial nestas singularidades. Este campo pode ter ainda um número finito de zeros em pontos regulares, que denotaremos por $\left\{b_{i}\right\}$. No entanto, a fórmula de Poincaré-Hopf se tornará:

$$
\chi(X)=(-1)^{n} \sum_{i} I\left(v, b_{i}\right)+\#\left\{a_{i}\right\}-\sum_{j} \chi\left(X \cap \partial V_{j}\right)
$$

onde $n$ é a dimensão da variedade e $V_{j}$ é uma bola fechada suficientemente pequena em torno de cada singularidade $\left\{a_{j}\right\}$ (note que $X \cap \partial V_{j}$ é uma variedade suave, pois $\partial V_{j}$ intercepta $X$ transversalmente, de acordo com [17]).

Esta fórmula pode ser justificada da seguinte maneira. Se removermos de $X$ cada $V_{j}$, teremos uma reunião disjunta de variedades suaves com bordo (contendo todos os zeros $\left.\left\{b_{i}\right\}\right)$. Como o campo $v$ é radial, o campo -v está saindo desta variedade com bordo, e daí podemos aplicar o teorema de Poincaré-Hopf original [16].

Ao reunir novamente a variedade com bordo com as vizinhanças retiradas, aparecem as duas últimas parcelas da soma.

Exemplo 10.1. Consideremos o campo a seguir na figura 8.

Notemos que, além do ponto singular $a$, o campo apresenta mais quatro zeros, $b_{1}$, $b_{2}, b_{3}$ e $b_{4}$, de índices, respectivamente, $-1,-1,1$ e -1 .

A intersecção de $X$ com uma vizinhança $V$ suficientemente pequena de $a$ consiste de 4 pontos, logo $\chi(X \cap V)=4$.

Aplicando a fórmula acima, obtemos:

$$
\chi(X)=(-1)(-1-1+1-1)+1-4=-1
$$

que é o esperado ao calcular a característica de Euler-Poincaré realizando uma divisão celular. 


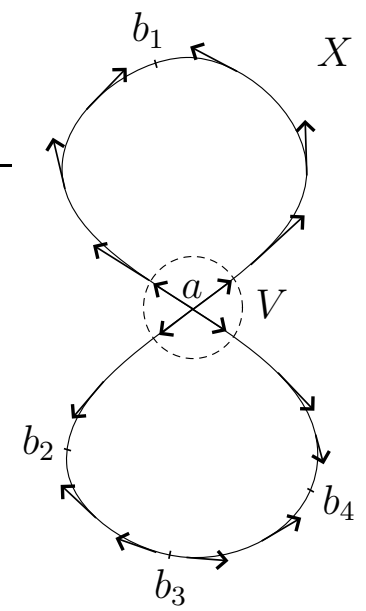

FIGURA 10.2: Campo na figura 8

Exemplo 10.2. Considere novamente o campo radial da Figura 10.1. Podemos calcular a característica a partir da nova fórmula: como o campo não tem zeros além de $a$ e a intersecção de uma vizinhança de $a \operatorname{com} X$ é reunião de duas circunferências disjuntas (característica 0), temos

$$
\chi(X)=(-1)^{2}(0)+1-0=1
$$

que novamente é o resultado esperado. 


\section{Referências Bibliográficas}

[1] Brasselet, J.P. and Schwartz, M.H., Sur les classes de Chern d'un ensemble analytique complexe, Astérisque 82-83 (1981), 93-147.

[2] Brasselet, J.P., Lê, D.T. and Seade, J., Euler obstruction and indices of vector fields, Topology, 2000.

[3] Brasselet, J.P., Classes características de variedades singulares, Notas didáticas 56, ICMC-USP, 2002.

[4] Brasselet, J.P., Poincaré-Hopf theorems on singular varieties - characteristic classes, Advanced School and Workshop on Singularities in Geometry and Topology, ICTP, Trieste, 2005.

[5] Buchweitz, R. and Greuel, G., The Milnor Number and deformations of complex curve singularities, Inventiones Math. 58 (1980), 241-281.

[6] Dimca, A., Singularities and topology of hypersurfaces, Springer-Verlag, 1992.

[7] Gibson, C.G., Wirthmüller, K., Plessis, A. and Looijenga E.J., Topological stability of smooth mappins, LNM 552, Springer-Verlag, 1976.

[8] Gonzalez-Sprinberg, G., L'obstruction locale d'Euler et le Théorème de MacPherson, Astérisque 82-83 (1981), 7-32.

[9] Greenberg, M.J., Lectures on algebraic topology, W.A. Benjamin, 1967.

[10] Guillemin, V. and Pollack, A., Differential topology, Prentice Hall, 1974.

[11] Gusein-Zade, S.M., Luengo, I. and Melle-Hernández, A., Topology of meromorphic germs and its applications, (Russian) Algebra i Analiz 11, $\mathrm{n}^{\circ} 5$ (1999), 92-99; translation in St. Petersburg Math. J. 11, n 5 (2000), 775-780.

[12] Lima, E.L., A característica de Euler-Poincaré, Matemática Universitária 1 (1985), 47-62.

[13] Lojasiewicz, S., Triangulations of semi-analytic sets, Ann. Scual. Norm. Sup. di Pisa, Série III, vol. 17, Fasc. 4, 1964.

[14] Lojasiewicz, S., Ensembles Semi-Analytiques, IHES Lecture Notes, 1965.

[15] Macpherson, R., Chern classes for singular algebraic varieties, Ann. Math. 100, n² (1974), 423-432. 
[16] Milnor, J., Topology from the differentiable viewpoint, Un. Press of Virginia, Charlottesville, 1965.

[17] Milnor, J., Singular points of complex hypersurfaces, Ann. of Mathematics Studies, Princeton Un. Press, 1968.

[18] Milnor, J., Characteristic classes, Princeton Un. Press, 1974.

[19] Miyazaki, H., Paracompactness of $C W$ complexes, Tohoku Math. J.4(1952), 309-313.

[20] Mond, D., Some remarks on the geometry and classification of germs of maps from surfaces to 3-space, Topology 26 (1987), 361-383.

[21] Munkres, J.R., Elements of algebraic topology, Benjamin/Cummings, 1984.

[22] Sebastiani, M., Sur la formule de Gonzalez-Verdier, Bol. Soc. Bras. Mat., Vol. $16 \mathrm{n}^{\circ} 1$ (1985), 31-44.

[23] Spanier, E.H., Algebraic topology, McGraw-Hill, 1996.

[24] Steenrod, N., The topology of fibre bundles, Princeton Un. Press, 1951.

[25] Vick, J.W., Homology theory: an introduction to algebraic topology, New York: Academic Press, 1973.

[26] Whitney, H., The self-intersecions of a smooth n-manifold in 2n-space, Ann. Math. 45 (1944), 220-246.

[27] Whitney, H., The singularities of a smooth n-manifold in (2n-1)-space, Ann. Math. 45 (1944), 247-293.

[28] Whitney, H., Tangents to an analytic variety, Ann. Math. 81 (1965), 496-549. 


\section{Índice Remissivo}

Anel de cohomologia, 9

Aplicação

de Gauss, 13

entre fibrados, 3

exponencial, 27

linear genérica, 74

Aplicações homotópicas, 12

Campo

de Hopf, 16

estratificado, 57

radial, 57

Categoria, 5

Ciclo algébrico, 73

Classe

diagonal, 29

dual, 28

fundamental, 24

de homologia, 30, 41, 71

Condições de regularidade, 50

Conjunto construtível, 67

Derivada, 1

Difeomorfo, 1

Dualidade de Whitney, 20

Excisão, 8

Fórmula

de Küneth, 30

de Lefschetz, 74

fibra, 3

Fibrado

de Nash, 69

de retas, 4

induzido, 3

normal, 4

produto, 3

tangente, 4

trivial, 3

universal, 21, 38

Função construtível, 67

suave, 1

Funtor contravariante, 5

Homogeneidade, 12

Isomorfismo de Thom, 24

Métrica

euclidiana, 4

riemanniana, 4

Modificação de Nash, 69

Naturalidade, 19, 24, 37

Orientação para fibrados, 23

para variedades, 12, 23

Plano tangente, 1

Poliedro, 14

Ponto regular, 11

Posição geral, 51

Produto Slant, 30

Recobrimento duplo, 25

Regra da cadeia, 2

Seção, 4

Seqüência exata, 7

Simplexo, 6

Soma de Whitney, 4

Teorema do Produto de Whitney, 19, 38

Toro pinçado, 15

Transversalidade, 51

Valor regular, 11

Variedade

algébrica, 49

com bordo, 11

de Stiefel, 20

irredutível, 49

Vizinhança tubular, 27 